\title{
WestVirginiaUniversity
}

THE RESEARCH REPOSITORY @ WVU

Graduate Theses, Dissertations, and Problem Reports

2014

\section{A Force Sensor Array for Space Applications}

Timothy L. Weadon

West Virginia University

Follow this and additional works at: https://researchrepository.wvu.edu/etd

\section{Recommended Citation}

Weadon, Timothy L., "A Force Sensor Array for Space Applications" (2014). Graduate Theses,

Dissertations, and Problem Reports. 628.

https://researchrepository.wvu.edu/etd/628

This Dissertation is protected by copyright and/or related rights. It has been brought to you by the The Research Repository @ WVU with permission from the rights-holder(s). You are free to use this Dissertation in any way that is permitted by the copyright and related rights legislation that applies to your use. For other uses you must obtain permission from the rights-holder(s) directly, unless additional rights are indicated by a Creative Commons license in the record and/ or on the work itself. This Dissertation has been accepted for inclusion in WVU Graduate Theses, Dissertations, and Problem Reports collection by an authorized administrator of The Research Repository @ WVU.

For more information, please contact researchrepository@mail.wvu.edu. 


\title{
A Force Sensor Array for Space Applications
}

Timothy L Weadon

Dissertation submitted to Benjamin M. Statler College of Engineering and Mineral Resources at West Virginia University

In partial fulfillment of the requirements for the degree of Doctor of Philosophy in Mechanical and Aerospace Engineering

\author{
Edward M. Sabolsky, Ph.D., Chair \\ Thomas Evans, Ph.D. \\ Kostas Sierros, Ph.D. \\ Dave Graham, Ph.D. \\ Darran Cairns, Ph.D.
}

Department of Mechanical and Aerospace Engineering

\author{
Morgantown, West Virginia \\ 2014
}

Keywords: space, geostationary earth orbit, harsh environment, capacitive, capacitance, permittivity, force, stress, pressure, sensor 


\section{ABSTRACT \\ A Force Sensor Array for Space Applications Timothy L Weadon}

The need for force feedback and spatial awareness of contact in harsh environment applications, such as space servicing, has been unsatisfied due to the inability of current sensor technology to resist environmental effects. In this work, capacitive sensors based on a porous polymer-ceramic composite structure were evaluated for potential use in future operations within robotic end-effectors, withstanding temperatures ranging from $-80{ }^{\circ} \mathrm{C}$ to $120^{\circ} \mathrm{C}$ and forces up to $350 \mathrm{kPa}$. A thin-film design is utilized to allow for ease of embedding, allowing sensors to be implemented into exciting robotic hardware with minimal intrusion, and protecting sensors from electron bombardment, radiation, and point concentrations from metal-on-metal contact. Furthermore, said embedding is proposed to protect against environmental effects including electron bombardment, radiation, atomic oxygen, and damage caused by point concentrations during metal-on-metal contact.

The novel sensor design optimizes constituent properties to maximize the response and reduce background noise, hysteresis, and thermal and mechanical drift. Selection of continuants and design parameters is presented explicitly, including synthesis and preparation of necessary materials and execution of processing methods. Qualification of the design is achieved through thorough dynamic, quasi-static, and long term static thermomechancial loading schedules ranging from -80 to $120^{\circ} \mathrm{C}$ and 20 to $360 \mathrm{kPa}$ with in-situ electrical acquisition. The final composition is also shown to meet necessary outgassing standards for in-orbit operations. Additional parameters are presented for selection of necessary substrate and electrode materials, further optimizing the applied technology.

An analytical model for pressure sensors is also constructed, predicting the capacitive response of a porous, polymer-ceramic composite under an applied pressure. Consisting of mechanical and dielectric counterparts, the iterative model is constructed in detail. The elastic modulus of the three-phase material is approximated by first considering only the polymer-ceramic composite mixture, and then incorporating porosity into the solid composite model. A new model has been developed for approximating the changing elastic modulus of porous polymers undergoing quasi-static compression, which induces the collapsing pores. Necessary material constants were obtained from experimental data published in literature. The permittivity of the paraelectric polymer matrix is modeled, accounting for piezodielectric effects imposed by external pressure and thermally induced stresses caused by substrate pinning. Similarly, the ferroelectric ceramic filler is modeled, considering changes in polarization caused by thermally induced phase transformations in the crystal structure. The final model is evaluated against experimental data, providing insight into composition and microstructure effects on the sensor response. 
To Joanna, Galilee, and Judah Weadon Soli Deo Gloria 


\section{Table of Contents}

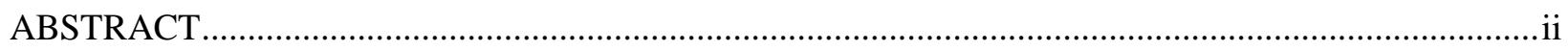

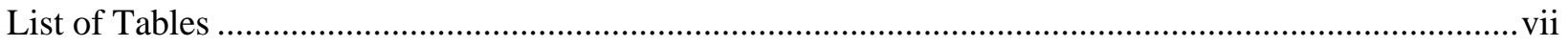

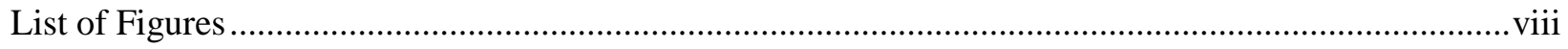

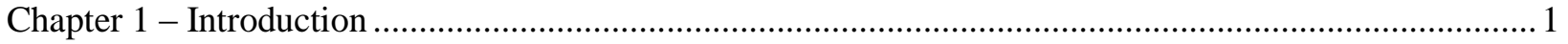

1.1. Statement of problem - the NASA Robotic Refueling Mission ..................................................... 1

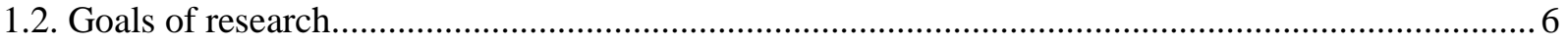

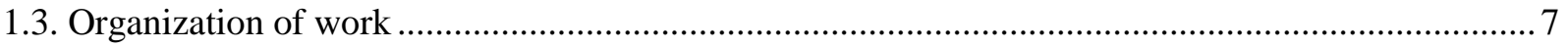

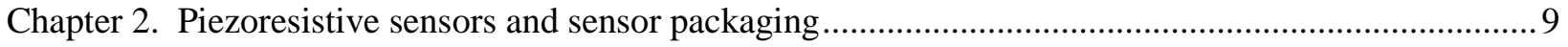

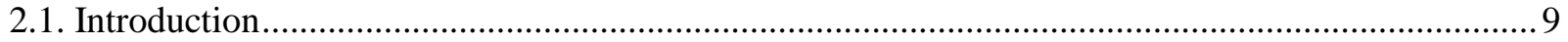

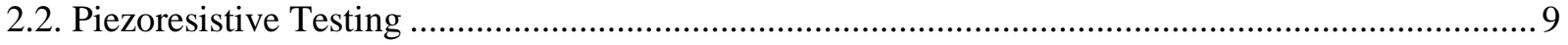

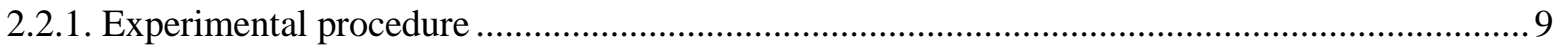

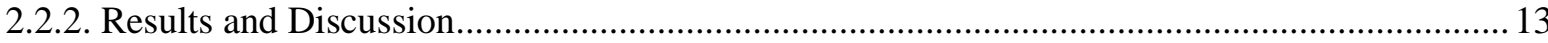

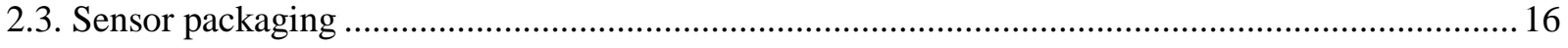

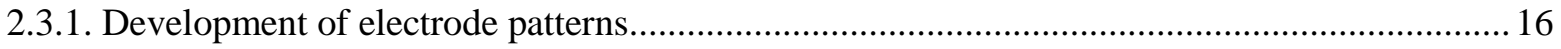

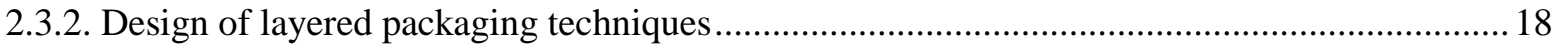

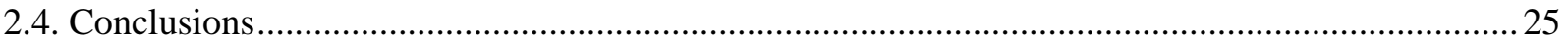

Chapter 3. Experimental Design of a Ceramic-Polymer Capacitive Force/Spatial Awareness Sensor

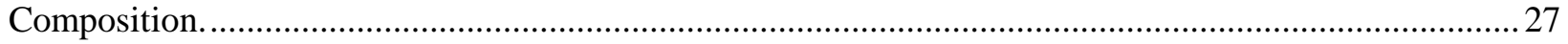

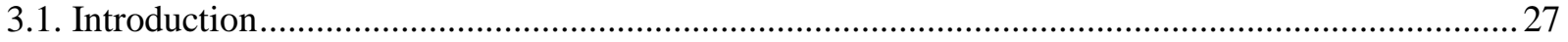

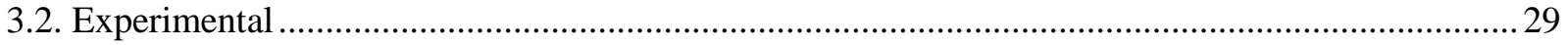

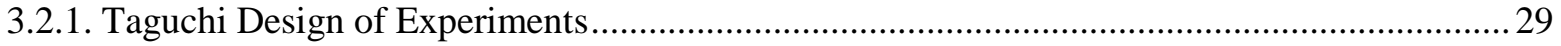

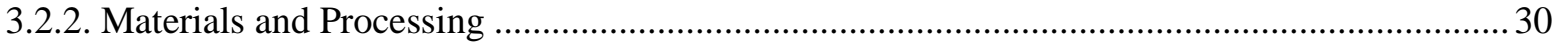

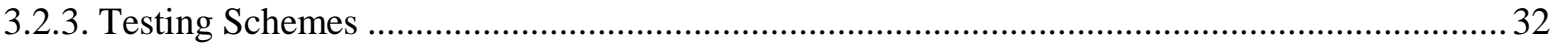

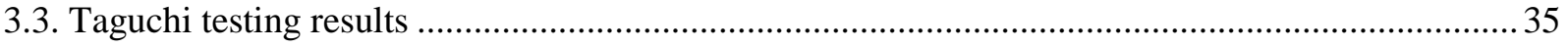

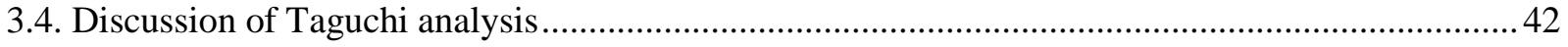

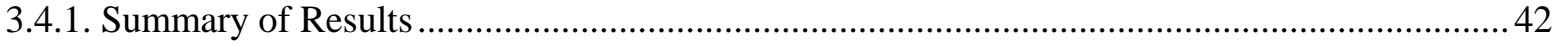

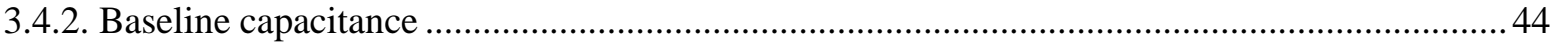

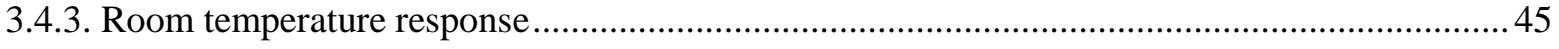

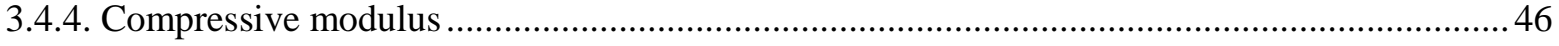

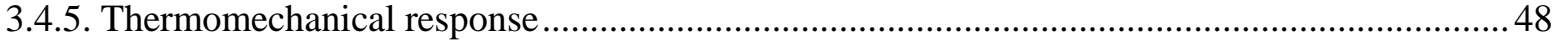

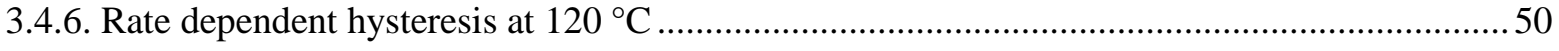

3.4.7. Thermomechanical drift.............................................................................................. 51

3.4.8. Characterization of thermal affects on capacitance............................................................52

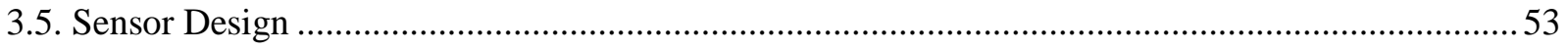




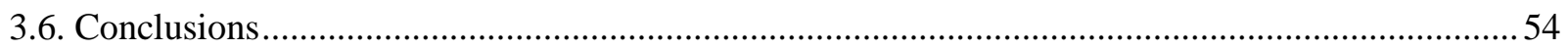

Chapter 4. An Analytical Model for Porous Polymer-Ceramic Capacitive Pressure Sensors ....................56

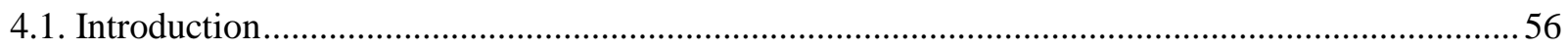

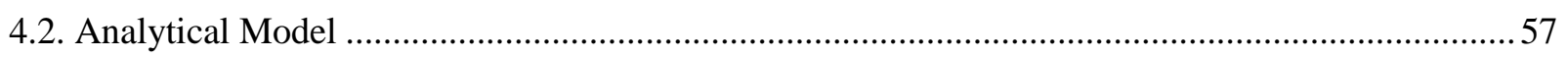

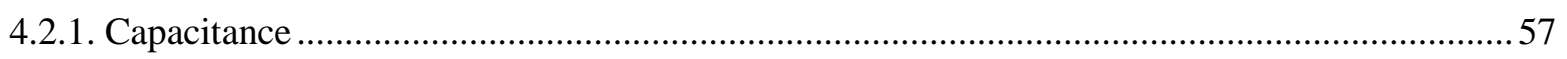

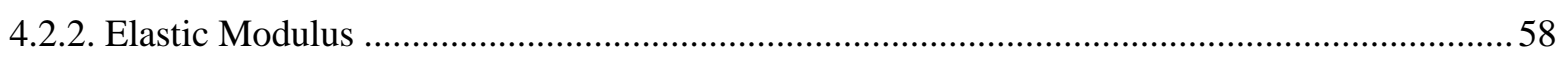

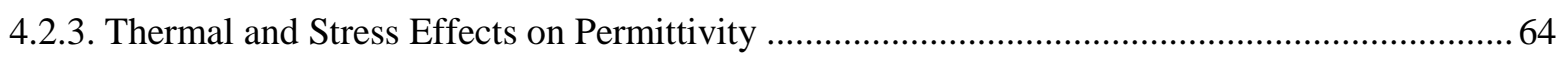

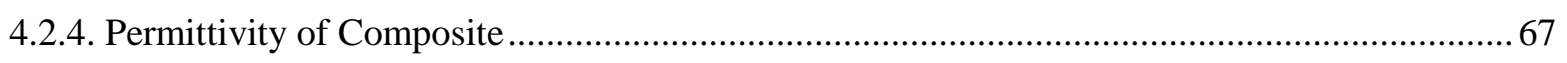

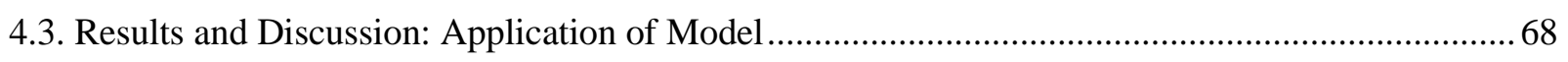

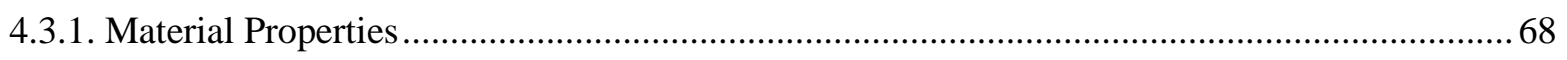

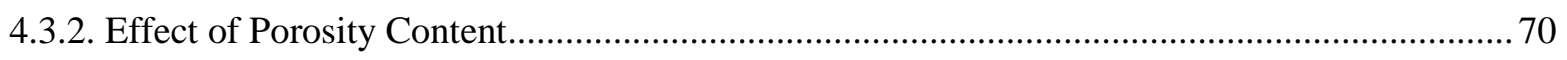

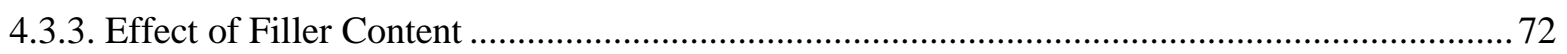

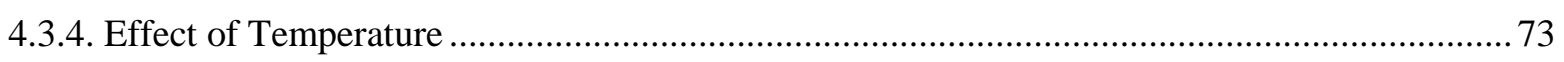

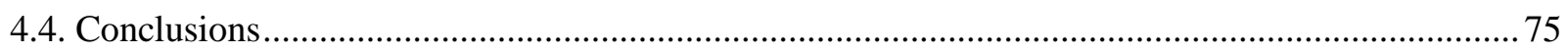

Chapter 5. Capacitive Force/Spatial Awareness Sensor for Robotic Space Servicing Applications ......... 76

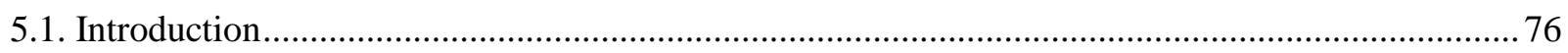

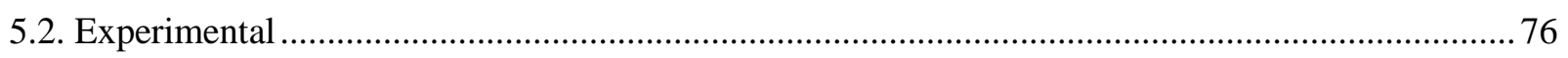

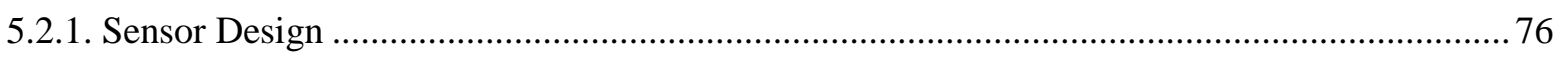

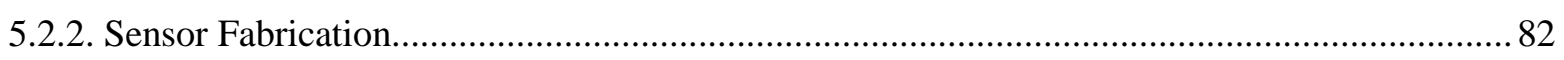

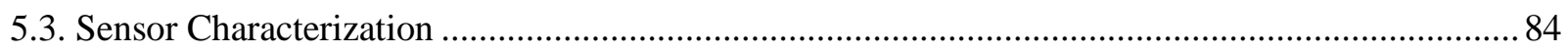

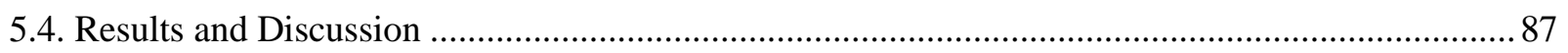

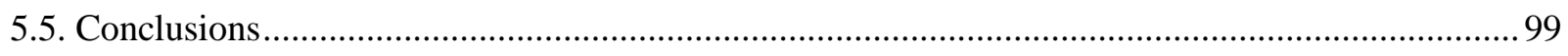

Chapter 6. Embedded sensor design and future work................................................................. 101

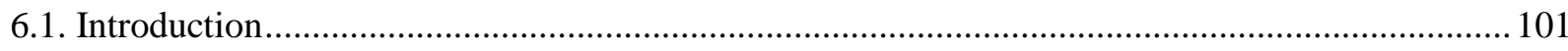

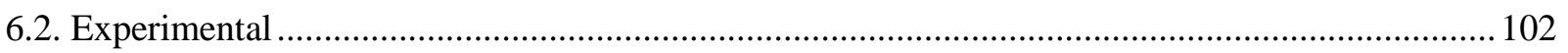

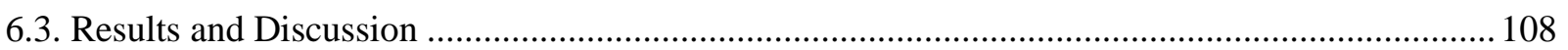

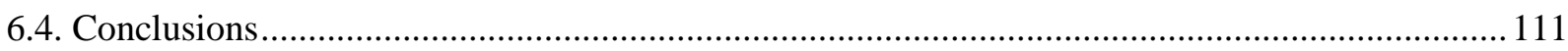

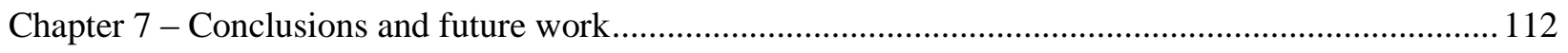

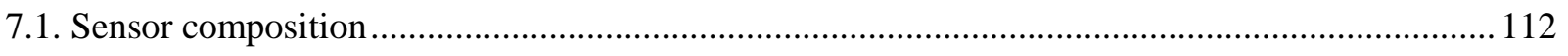

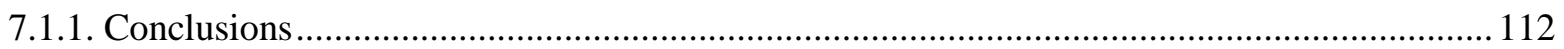

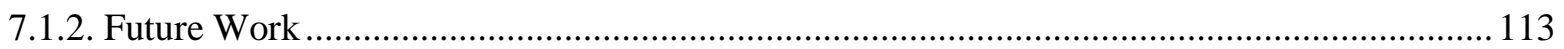

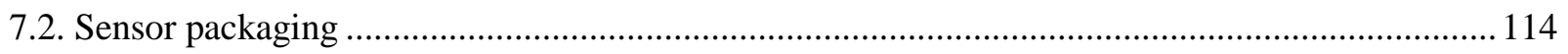

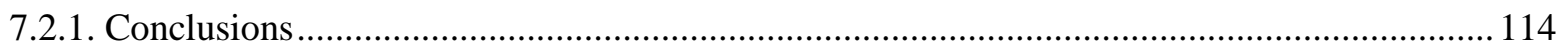

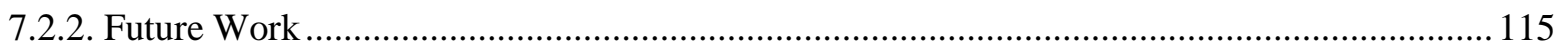

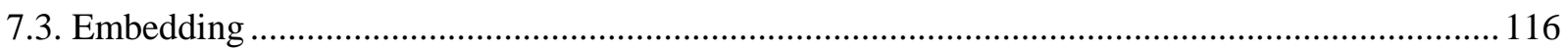




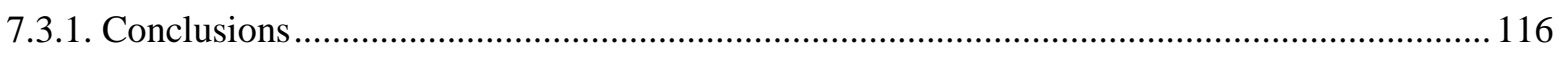

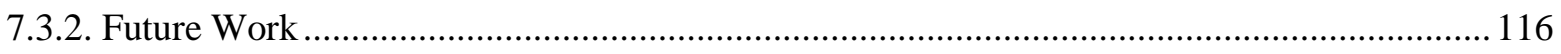

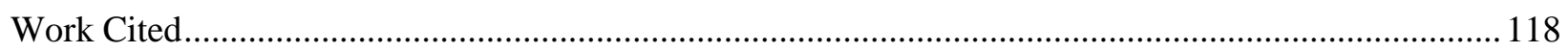




\section{List of Tables}

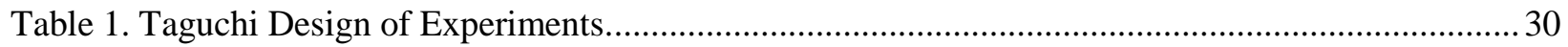

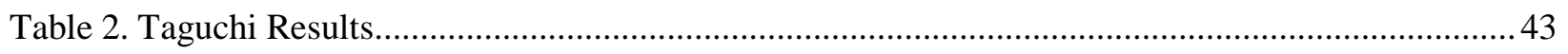

Table 3. Composite composition versus average sensor response .......................................................46

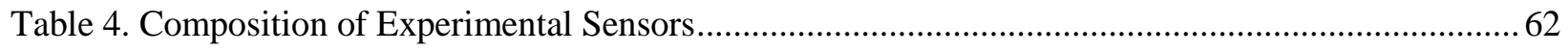

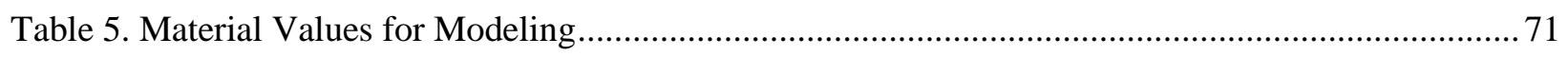

Table 6. Outgassing data for BTO and PZT filled compositions........................................................ 98 


\section{List of Figures}

Figure 1. SEM micrograph of carbon black used as filler for piezoresistive sensors.

Figure 2. Thermomechanical testing setup: a) DMA instrument, b) grip configuration for testing with in situ electrical acquisition, c) compliant layer used to ensure homogenous pressure distribution. 11

Figure 3. Circuit used to optimize sensor feedback resolution. .12

Figure 4. FlexiForce piezoresistive sensors purchased from Tekscan. 12

Figure 5. Response (voltage) to an applied stress for a sensor with 5 vol\% carbon filler. 13

Figure 6. Response (voltage) to an applied stress for a sensor with 10 vol\% carbon filler. .13

Figure 7. Response (voltage) to an applied stress for a sensor with 20 vol\% carbon filler. 14

Figure 8. Response (voltage) to an applied stress for a sensor with $30 \mathrm{vol} \%$ carbon filler. 14

Figure 9. FlexiForce sensor response (voltage) under a ramp load up to $350 \mathrm{kPa}$ for temperatures of 25 ${ }^{\circ} \mathrm{C}, 40{ }^{\circ} \mathrm{C}, 80{ }^{\circ} \mathrm{C}$, and $120{ }^{\circ} \mathrm{C}$.

Figure 10. Sensor package design, showing protective polymer films and electrode patterns.

Figure 11. Thin film piezoresistive sensors adhered by a) paper based tape, b) aerosol based glue, c-d) polymer based tape.

Figure 12. Electrode cracking caused by deformation of the polymer.

Figure 13. Aluminum mold for casting an embedded sensor prototype with hillocks to ensure first contact on sensor face.

Figure 14. Embedded sensor prototype, showing a thick-film sensor embedded within a rubber matrix.. .21

Figure 15. Demonstration of plug-and-play sensor use: a) applying sensor and connecting to DAQ, b) sensors used for real time contact information with graphic user interface.

Figure 16. Sensor package: a) deposition schedule for sensor package fabrication, b) fully fabricated sensor package, c) thickness as compared with a penny, d) flexibility of sensor package.

Figure 17. A mechanical stimulation (force) applied to show the sensor response (capacitance) both as a function of time. The delay between stimulation and response reveals a rate dependent hysteresis.

Figure 18. Thin film sensor composition being retrieved from a water bath after being released from the glass substrate. 
Figure 19. Polymer binder and organic modifier molecular architectures.

Figure 20. SEM micrographs of the ceramic particles (a) milled PZT, (b) as-received PZT, (c) as-received BTO, (d) calcined BTO.....

Figure 21. Thermomechanical testing setup: a) DMA instrument, b) grip configuration for testing with in situ electrical acquisition, c) compliant layer used to ensure homogenous pressure distribution. 33

Figure 22. Applied stress versus time schedule used for the sensor response characterization. .33

Figure 23. Applied stress versus time schedule used for thermomechanical testing at (a) low temperature and (b) high temperature.

Figure 24. Room temperature capacitance of minimally loaded (40 kPa) sensor compositions at $200 \mathrm{kHz}$.

Figure 25. Room temperature capacitive response of sensor compositions at $200 \mathrm{kHz}$. .36

Figure 26. Room temperature capacitive response of ceramic free sensor compositions at $200 \mathrm{kHz} . \ldots . . .37$

Figure 27. SEM images of the top surface of sensor materials A-H. .37

Figure 28. Stress versus strain at room temperature and stressed at a rate of $120 \mathrm{kPa} / \mathrm{min}$ for Sensors A-H.

Figure 29. Applied stress versus the $\%$ strain and derivatives for determination of the transition point between low strain and high strain deformation of Sensor H. Plots shown are: a) splitting the angle between the two slops b) transition in the first derivative.

Figure 30. Thermomechanical loading schedule and response of sensor material A. 40

Figure 31. Sensor responses across the full temperature range.

Figure 32. Capacitance and dielectric loss as a function of temperature (measured at $200 \mathrm{~Hz}$ ) for composite sensor materials A-H.

Figure 33. Capacitance and loss tangent as a function of frequency for sensor materials A-H at room temperature.

Figure 34. Capacitance response $\left({ }^{\circ} \mathrm{C}\right)$ as a function of temperature for sensor materials containing PZT (average of Sensors A, B, E, F) and BTO (average of Sensors C, D, G, H) .......................................... 48

Figure 35. Sensor prototype fabricated to demonstrate use of the proposed smart film technology. .........54

Figure 36. SEM micrograph of sensor A (composition details listed in Table 4) ...................................57 
Figure 37. The normalized elastic modulus of the porous material plotted against the change in porosity under an applied load $0 \rightarrow 350 \mathrm{kPa}$.

Figure 38. Summary of equations used for modeling the composite sensor.

Figure 39. Capacitance change as a function of pressure measured and modeled for various sensor compositions.

Figure 40. Sensor capacitance change as a function of pressure for sequential porosities, quantifying the effect of porosity on characteristics of the sensor response.

Figure 41. Sensor capacitance change as a function of pressure for sequential filler contents, quantifying the effect of filler loading on characteristics of the sensor response.

Figure 42 . The permittivity of the matrix and filler constituents plotted as a function of temperature...... 74

Figure 43. Modeled and experimental capacitance change of the three-phase sensor as a function of temperature.

Figure 44. XRD analysis of synthesized PZT, showing estimated rhombohedral (zirconium rich) and tetragonal (titanium rich) phase distribution.

Figure 45. Particle size distribution of synthesized PZT obtained by dynamic light scattering, plotted against the number of particles.

Figure 46. SEM micrograph of a) purchased BTO, and b) synthesized PZT, showing size and aspect ratio of particles.

Figure 47. Preparation of specimens for cross-sectional observation using SEM.

Figure 48. SEM micrograph of thin-film cross section for (a) BTO filled composition and (b) PZT filled composition, and top surface of (c) BTO filled composition and (d) PZT filled composition.

Figure 49. Thermomechanical testing setup: a) DMA instrument, b) grip configuration for testing with in situ electrical acquisition, and c) compliant layer used to ensure homogenous pressure distribution........85

Figure 50. DMA configuration for simultaneous cyclic loading of 5 specimens, using silicone to distribute stress across the sensor face.

Figure 51. The sensor response (capacitance) to a mechanical step load from $0 \mathrm{kPa} \rightarrow 360 \mathrm{kPa}$ as a function of temperature for BTO filled composite specimens.

Figure 52. The sensor response (capacitance) to a mechanical step load from $0 \mathrm{kPa} \rightarrow 360 \mathrm{kPa}$ as a function of temperature for PZT filled composition specimens.

Figure 53. Sensor response (capacitance) of PZT filled composite specimen 3 at $-80{ }^{\circ} \mathrm{C}$, exhibiting sporadic behavior over the course of 10 step loads from $20 \mathrm{kPa} \rightarrow 360 \mathrm{kPa}$. This behavior was caused by partial leakage currents between PZT particles within the bulk 
Figure 54. A piece of Kapton ${ }^{\circledR}$ film was used as a substrate, with platinum electrodes deposited by DC sputtering, and PZT filled sensor composition cast using a mayer rod.

Figure 55. Capacitive response of five specimens for each sensor composition under quasi-static load application at room temperature: a) BTO filled compositions, b) PZT filled compositions, and c) unmodified Arathane 5750.

Figure 56. Ten loading runs performed on specimen 1 for each sensor composition showing the capacitive response under quasi-static load application at room temperature: a) BTO filled compositions, b) PZT filled compositions, and c) unmodified Arathane 5750.......

Figure 57. Sensor response (capacitive) of BTO filled sensor composition before and after undergoing 100,000 step loading cycles from $20 \mathrm{kPa} \rightarrow 360 \mathrm{kPa}$

Figure 58. Sensor response (capacitive) of PZT filled sensor composition before and after undergoing 100,000 step loading cycles from $20 \mathrm{kPa} \rightarrow 360 \mathrm{kPa}$

Figure 59. Sensor response over time for BTO filled (left) and PZT filled (right) compositions, with an applied static load of $360 \mathrm{kPa}$.

Figure 60. Normalized sensor response over time, with an applied static load of $360 \mathrm{kPa}$.

Figure 61. SEM micrograph showing cross-section of PZT filled composite specimen 1.

Figure 62. Proposed sensor package for applied use of sensor composition.

Figure 63. Proposed technique for minimally intrusive embedding in existing end effectors.

Figure 64. A marman ring mounted on a mock satellite, with an automated robotic space simulator for lab demonstrations of proposed in-orbit tasks.

Figure 65. NASA GSFC RRM Gripper tool for docking robotic equipment on the satellite marman ring for refueling and repair of satellites in earth orbit.

Figure 66. Gripper tool prototype, with dimensions matching the docking area shown in orange in figure 65. In this figure the blue represents the aluminum bulk material, and the red represents cavities machined to a depth of 0.125 " providing area for embedded sensors.

Figure 67. Gripper tool prototype machined from aluminum, with end milled cavities for embedding sensors.

Figure 68. Kapton ${ }^{\circledR}$ film substrate, with platinum electrodes and BTO filled sensor composition cast using a mayer rod $(9.5 \mu \mathrm{m})$. The sensor composition was wiped from the electrode tips, providing access to mount silver micro-wires. 
Figure 69. The embedded sensor prototype, shown with a cutaway view to reveal the sensor package, silicone compliant layer, and titanium foil cover.

Figure 70. The fully complete marman ring gripper tool prototype, hosting two sensors.

Figure 71. The three different testing configurations: 1) bare sensor package, 2) embedded sensor with rubber compliant layer, and 3) fully embedded sensor with metal foil cover.

Figure 72. The Shimadzu testing setup with stock compressive clamps, the lower (static) clamp having been prepared with two compliant layers to prevent accidental overloading from metal-on-metal contact.

Figure 73. A total of four sensor specimens (a-d) were tested in each of the three testing configurations. Each configuration is plotted for a single specimen, showing the difference in the sensor response (capacitance) under an applied stress.

Figure 74. Sensor response (capacitance) as a function of applied stress (force/area) for specimen A, loaded as a bare film between two plates of metal.

Figure 75. Sensor response (capacitance) as a function of applied stress (force/area) for specimen A, loaded as an embedded film within the gripper tool prototype with a rubber compliant layer.

Figure 76. Sensor response (capacitance) as a function of applied stress (force/area) for specimen A, loaded as an embedded film within the gripper tool prototype with a rubber compliant layer and titanium foil.

Figure 77. Cross sectional view of proposed embedding design for pixelated metallic contact points in a gripper tool, providing distribution of point forces and protection from overload through a compliant material. 


\section{Chapter 1 - Introduction}

\subsection{Statement of problem - the NASA Robotic Refueling Mission}

The need for on-orbit servicing of satellites has recently been recognized on an international level and the benefits to society and satellite manufactures is evident. In 2010 the satellite industry posted revenues of $\$ 168.1$ billion, with an annual growth averaging above 11\% since 2005 [1]. Projections show that the demand for new satellite launches is steady [2], and statistics show rapidly increasing on-orbit failures "to worrying levels" [3].

Advanced robotic technology for space operations increases the capability to address common on-orbit decommissioning or unplanned anomalies which can render a satellite as inoperable. These anomalies include the depletion of a fuel source, failure to achieve or maintain proper orbital position, and failure to deploy simple mechanisms. The effects of on-orbit failures or anomalies has statistically been evaluated to reveal an approximate annual loss of \$48 million for typical communications satellites [3]. Current engineering efforts are in process to prove the capability to robotically refuel, repair, and relocate satellites in need of service. [4].

Spatial awareness of is essential for remote on-orbit robotic servicing. Current space robotic systems lack the required force and spatial information necessary for preventing damage to hardware by misguided contact, over gripping, or premature contact of robotic end effectors [5]. Commercially available sensor arrays are incompatible for space use because of electrical and volumetric restraints, and environmental conditions [6]. Current robotic systems utilize a range of technologies including force/torque sensors, strain gauges, motor current calculations, and flexibility modeling [7]. While these technologies quantify forces occurring at the end effector, they fail to report any information about contact location, force magnitude, or slipping within the end effector during grappling procedures.

More advanced technologies have recently been proposed for on-orbit servicing including capacitive proximity sensors (the capaciflector) for contact prediction and embedded spring elements for force quantification within end effectors [6,8]. The capaciflector was not used for space applications, due to inadequate levels of resolution. Furthermore, spring elements experience high drift, and were not designed for complex tooling systems making integration into existing designs implausible. Additionally, recent advanced force sensor technologies discussed in literature demonstrate the use of silicon-based or micro-electromechanical (MEMs) sensors which are not suitable for high-force, high abrasion, harsh environment applications [9]. 
In order to meet the demands of on-orbit servicing applications, a number of environmental, mechanical, electrical, and volumetric restraints must be considered. Environmental conditions are encompassed by vacuum as low as $10^{-7}$ torr, temperatures ranging from $-80{ }^{\circ} \mathrm{C}$ to $120{ }^{\circ} \mathrm{C}$, electron/ion bombardment, and radiation [10-11]. Charging and radiation factors will be addressed by embedding the sensor materials within metal end-effectors. Thus, sensors must also be easily embedded into new and existing robotic hardware, requiring the sensing material to be thin, flexible, and easily customized to meet design requirements. Finally, sensors must have minimal power, hardware, and real estate requirements.

Many sensor architectures have been developed in literature, allowing for dispersion of point concentrations and acquisition of normal and shear stresses across multiple axes simultaneously. This area has been thoroughly studied, and is not of immediate value for this work. In this work all demonstrated applications assume contact surfaces will be larger than the sensor face, so that sensors are not partially loaded. Contact forces are also assumed to be planar, resulting in a stress occurring across the surface area of the sensor face. The surface roughness of these planar contacts (line and point concentrations) is assumed not to exceeding $150 \mu \mathrm{m}$ in depth. These assumptions are consistent with the proposed metal-on-metal grappling of machined parts. Normal compressive stresses will be quantified in this work, with a maximum stress of $350 \mathrm{kPa}$. The proposed use of the sensor system is broad, and includes static and dynamic applications with a variety of contact dynamics.

Thin-film force and spatial sensors are most commonly fabricated using resistive, piezoelectric, and capacitive activities for sensing. In addition to requiring charge amplifiers, piezoelectric sensors have a known drift in the sensor output, requiring power and surface area for additional circuitry when used in static applications. This limits their potential application in on-orbit servicing projects [13-14].

The piezoresistive sensor technology has been studied for many years, and is well understood. In 1925, Bridgman showed the first correlation between stress and resistance in polycrystalline metals, introducing the piezoresistive effect [15]. Integrated piezoresistive sensors were fabricated by Tufte et al. in 1962, using silicon diaphragms to measure changes in resistance as a function of pressure. Previous to this work, single crystal metal strain gauges were glued to surfaces. This method introduced excessive hysteresis and creep, as well as having low resolution [16]. Work by Malliaris et al. in 1970 showed that adding conductive particles to a resistive matrix revealed a critical transition point, where the composite begins to conduct electrically above a certain threshold of filler loading [17]. Soon after, Bueche showed that conductivity in these materials was sensitive to temperature near the polymer melting temperature, allowing them to be used as electrical temperature sensors [18]. In 1975, Harden filed for a patent, which includes pressure versus resistance data for elastic materials filled with conductive particles [19]. 
Piezoresistive sensors are currently among the most used and furthest developed sensors for force response in micromachined devices [20]. Applications rage widely, including bio-compatible sensors for use in human applications, online feedback for autonomous control of robotic systems, and high resolution 2D sensor arrays [21-23]. However, these sensor systems are known to experience drift, hysteresis, creep, and a loss in sensitivity [24-25]. In this work, piezoresistive sensors were used as a method for testing and evaluating flexible electrode deposition, sensor packing, and fabrication of 0:3 composite sensor materials. This allowed for development of fabrication techniques in parallel with the design of a more complex capacitive sensor technology.

This work proposes the use of capacitive sensor technology for the fabrication of force and spatial sensor arrays as they exhibit high sensitivity, low drift, reduced temperature dependence, and require low power consumption compared with piezoresistive sensors [26-27]. These sensors simply quantify the difference in stored charge of the sensing materials as a function of strain, based on the equation of capacitance:

$$
C=\frac{\varepsilon_{r} \varepsilon_{0} A}{d}
$$

where $C$ is capacitance, $\varepsilon_{r}$ is the relative permittivity (dielectric constant), $\varepsilon_{0}$ is the permittivity of free space, $A$ is the area, and $d$ is the thickness. Capacitive force sensors function by characterizing the relationship between an external pressure and a change in capacitance caused by the introduction of strain $(\Delta d)$. This relationship can either be experimentally modeled or predicted mathematically using the compressive modulus of the sensor. The sensor response is defined as the change in capacitance caused by an applied load, where $\epsilon$ is strain, $E$ is the elastic modulus, and $\sigma$ is the applied stress.

$$
\Delta C=\frac{\varepsilon_{r} \varepsilon_{0} A}{d_{1}(1+1 / \epsilon)}=\frac{\varepsilon_{r} \varepsilon_{0} A}{d_{1}(1+E / \sigma)}
$$

Historically, capacitive sensors were fabricated using air as the dielectric material between two electrodes. In an effort to simplify fabrication of the sensors, a thick film design was implemented which allows for simple printing of the electrodes [26-28]. Additional improvements were made by introducing high dielectric ceramics into the sensor films in order to enhance the capacitance, and thus the sensitivity for small sensor feature sizes [29]. Ceramics are the most prevalently used material in the fabrication of capacitors because of their high dielectric constant. Unfortunately, ceramic materials are not conducive 
for use in flexible, thick film capacitive sensors due to their high elastic modulus preventing high strain levels.

The alternative use of ceramic-polymer composites instead of pure ceramics provides a means for attaining a high dielectric constant while modifying the mechanical compliance of the material for use in capacitive sensor applications. Such a composite should consist of isolated micro/nano-ceramic particles within a polymer matrix to ensure consistent and homogenous mechanical and electrical properties. This design was pioneered by Newnham et al., where connectivity of the microstructure was termed as a 0:3 composite. This notation denotes a filler phase with no interconnectivity in all three dimensions (0interconnectivity) within a matrix exhibiting three-dimensional interconnectivity (3-interconnectivity) [30].

Most flexible force sensor technologies originated in the biomedical research area and lack the design criteria for use in more extreme temperature environments and cycling conditions. Therefore, many of these sensor platforms are not suitable for applications such as the on-orbit space servicing application previously discussed [27-29]. The aim of this work is to initiate research on flexible, capacitive force sensors based on a 0:3 composite design for use in harsh environments, specifically aiming the architecture towards a film that may be embedded below a thin metal skin on current space servicing robotic end effector designs. The thickness criteria for these sensors is defined by the need to embed the active sensor material (with patterned interconnect leads) within the end effector in order to protect the sensor from abrasion, and energy bombardment.

Selection of the active composite materials (ceramic dielectric and organic binder) was purposely chosen to represent a classification of materials that would be representative of those that would be demeaned space qualified. These material sets, and the methods and architecture used for the materials, act as a standard baseline for the future use of space qualified materials in future work. Furthermore, the scope of this work included the design and implementation of characterization and testing procedures necessary for qualifying electromechanical and thermomechanical responses of the sensor materials in the thermal conditions defined by in-orbit robotic operations. Evaluation criteria included the size constraints (for ease of embedding in existing metal parts), electrical, mechanical, and thermal properties affected by the material phase transitions and microstructure variables.

Currently capacitive sensors are used for a great variety of applications. Large capacitive sensor arrays are used in most touch screen technologies, and new high resolution arrays have been developed for replicating the force and spatial awareness of human skin [31]. Many sensors are also implanted in humans and animals for biometric applications, and used for observing hand gestures or translating hand motion to written language [32-35]. Recently the finite size, low cost, and high accuracy of these sensors 
has also led to use in structural health monitoring for a variety of civil and mechanical applications [3637].

As various polymer-ceramic composites are manufactured, using a diverse array of materials and processing methods, results can vary significantly. Obvious composition variables include the intrinsic properties of the polymer and ceramic materials, as well as, the volume fraction of the materials. However, these materials inherently contain a number of non-linear mechanical, electrical, and thermal attributes. The bulk material properties of composites become increasingly complex, as they are not linearly related to the volume fraction of the constituents. Further yet, the unintentional introduction of porosity (an additional phase) into the composite during processing has been found to unexpectedly alter materials properties [38-39]. This has often led to errors in calculating the basic capacitance of an unloaded material in a controlled environment [40-41].

An effort to obtain a better understanding of design variables has often led to analytical modeling, and finite element analysis (FEA) using computer aided designs (CAD) in recent literature. Various capacitive pressure sensors based on an open hollow membrane, or closed air-filled cavity, have been designed using these theoretical approaches with great success [32,42-44]. More complex PVDF - PZT (lead zirconium titanate) composites have also been modeled to predict the effect of PZT content on the permittivity, dielectric loss, piezoelectric coefficient, and elastic modulus [45-47]. Theoretical predictions of the piezoelectric effect were also made, showing the two-phase composites response to temperature [48-49]. However, models for predicting thermo-mechanical loads on porous polymer-filled composites are lacking.

Packaging of the flexible force sensor arrays has been developed far beyond the needs of this work by such prestigious groups as Harvard, Berkeley, and Stanford [50-52]. However, these designs cannot withstand the high loads and harsh environments required in this work. For this reason, a new more robust design is necessary. Sensor packaging in this work is also focused on simplification of processing methods, improvement of sensor response, and ability to be embedding in metallic or composite substrates. Embedded sensors in composite aerospace applications have been shown to introduce stress concentrations which weaken the structure [53]. Adding sensors to robotic gripper often requires remanufacturing the gripping assembly, or implementation of bulky sensor packages [8,54].

The embedding of sensor arrays in aerospace grade materials, such as Ti-6Al-4V alloy and a cyanate ester / carbon fiber composites, has proven to significantly reduce the strength of the materials [53]. For applications such as the one currently being considered, this loss in strength is caused by the introduction of a void space surrounding the embedded array, creating stress concentrations which result in crack propagation within the material. As a result, significant alterations to the original end-effector 
design will be required to maintain its strength and rigidity unless a new method for embedding is determined.

NASA's work on the robonaut has suggested packing sensors within a large, mulit-layered glove which will fit onto the robotic grippers, shown in figure 1 [55]. Designs such as this lack the ability to be incorporated into designs having tight clearance requirements or autonomous applications requiring minimal displacement after initial contact is made, and do not protect the sensor from overloading. This work introduces sensor packaging techniques allowing for numerous embedded approaches. It also proposes embedding techniques for both new and existing gripper designs.

\subsection{Goals of research}

The primary and overriding goal of this work is to accurately and consistently supply force and spatial information within end effectors. Achieving this task in geostationary earth orbit, under high gripping forces and metal-on-metal contact increases the need to be precise and intentional with all aspects of sensor design, sensor packaging, and embedding. The achievement of this goal was performed in this thesis by accomplishing the following tasks:

- An evaluation of piezoresistive sensors was completed to identify the shortcomings of the technology, and outline necessary improvements for use of the technology force quantification applications.

- Capacitive type force sensor compositions were evaluated using a through and extensive experimental matrix, complimented by a comprehensive literature review. This work provided the working mechanisms linking micromechanical, molecular, electrical, and thermal properties to specific attributes of the sensor character, such as noise, drift, hysteresis, and sensitivity.

- A complete analytical model was designed and implemented in Matlab®, using finite iterations to predict the sensor response (capacitance) as a function of applied stress. This model allowed theoretical designs to be evaluated computationally, furthering the capacitive sensor design.

- Using conclusions from an experimental design, a literature review, and analytical modeling an optimized capacitive sensor composition was proposed. The realization of the proposed design was achieved through synthesis of new materials and alterations to processing methods. The resulting material was characterized to demonstrate various attributes of the sensor character, including cyclic loading, long-term static loading, and thermal effects. 
- Numerous sensor packaging techniques were explored, presenting various approaches for different applications. The role of sensor packaging on the sensor response was also discussed, identifying sources of error which can be introduced if sensor packaging is not appropriately carried out.

- An embedding process for the NASA RRM marman ring gripper tool was successfully designed and carried out. Characterization of the proposed design demonstrated the importance of each embedding constituent, which in combination were shown to distribute point loads and quantify contact stresses in grapping procedures experience metal-on-metal contact.

\subsection{Organization of work}

This work is composed of seven chapters, which collectively introduce, examine, and summarize the aforementioned research objectives. The first chapter presents the need for robotic space servicing missions, and the motivation to fulfill the said need. The necessity of quantifying force magnitude, and the spatial mapping of forces for this mission are presented. This chapter also presents past work in the area of force sensor arrays, making use of a literature review to present a number of topics pertaining directly to various aspects of the sensor design and implementation.

The second chapter investigates the potential use of piezoresistive sensor technology to achieve the desired force and spatial information. Summarized therein is experimental testing, and a list of shortcomings of piezoresistive technology which must be overcome for the technology to provide accurate and reliable quantitative force information. Additionally, this chapter presents much progress on the topic of sensor packaging.

The third chapter presents a large experimental matrix, correlating the sensor properties with variations in the sensor response for capacitive type sensors. This work is complimented by a literature review, which identifies the working mechanisms which connects the sensor properties with the sensor response. The chapter is concluded by identifying limiting factors for sensor parameters and proposing an optimal sensor design. Chapter 4 furthers the design of the sensor composition, using analytical modeling to predict the sensor response (capacitance) as a function of applied stress. This chapter provides qualitative information concerning the role of volume loading for each composite sensor constituent, as well as predicting outcomes for specific material properties of various constituents.

In the fifth chapter, the optimized parameters from the third and fourth chapter are functionalized for the realization of a robust capacitive sensor composition. In some instances custom materials were synthesized to meet design needs, and the sensor design was further improved by considering processing 
variations on the sensor response. After fabrication, sensors were characterized to demonstrate the improved properties (increased sensitivity, decreased error).

Embedding of the sensor array was performed in the sixth chapter, as well as characterization of the embedded sensor. This work provided information pertaining to various aspects of the embedding process, such as the introduction of a silicone compliant layer and a titanium foil cover. The primary achievements of this work are summarized in the last chapter (chapter 7). This chapter also includes a list of future work, which the author has proposed for further improvements of the work. 


\section{Chapter 2. Piezoresistive sensors and sensor packaging}

\subsection{Introduction}

In an effort to fully evaluate piezoresistive sensor technology, both custom made and industrially manufactured sensors were characterized. First a simple polymer-filler material was fabricated to show the complexity of microstructure variables associated with piezoresistive materials. The purpose was to demonstrate various sources of error which must be carefully considered when using piezoresistive technology in force sensing applications. By identifying and improving sources of error, piezoresistive technology has potential for many new force applications. The second sensor was purchased from industry, having properties finely tailored to maximize response and minimize sources of error. These sensors were used to demonstrate thermal effects on the microstructure of piezoresistive sensors.

\subsection{Piezoresistive Testing}

\subsubsection{Experimental procedure}

The sensor construction was initiated by the fabrication of a piezoresistive ink composition. Based on work by Chang et al., the polymer matrix consisted of a 85 vol\% PVDF (Alfa Aesar, 500,000 g/mol MW) polymer binder matrix with 7.5 vol\% BPA (bisphenol A, Alfa Aesar, $228.29 \mathrm{~g} / \mathrm{mol} \mathrm{MW}$ ) used as a modifier, and 7.5 vol\% DEGBE (diethylene glycol monobutyl ether, Alfa Aesar, $162.23 \mathrm{~g} / \mathrm{mol} \mathrm{MW}$ ) used to assist as a solvent and dispersant for the organics [56]. A carbon black micro-powder was used as the conductive filler. This filler had a large particle size distribution, and thin, brittle nature are shown in a scanning electron microscope (SEM) micrograph in figure 1. The conductive filler was varied from 5 vol\% to 30 vol\% to adjust the sensor range and sensitivity, with the remaining portion being the polymer matrix. The inks were mixed by first dispersing the modifier and carbon black in the solvents, then adding the polymer binder with the assistance of a sonication wand. The solvent solution consisted of a 1:1 volume ratio of N-Methyl-2-pyrrolidone (nMP) and dimethylformamide (DMF), with a 25\% solid loading in the final ink solution. 


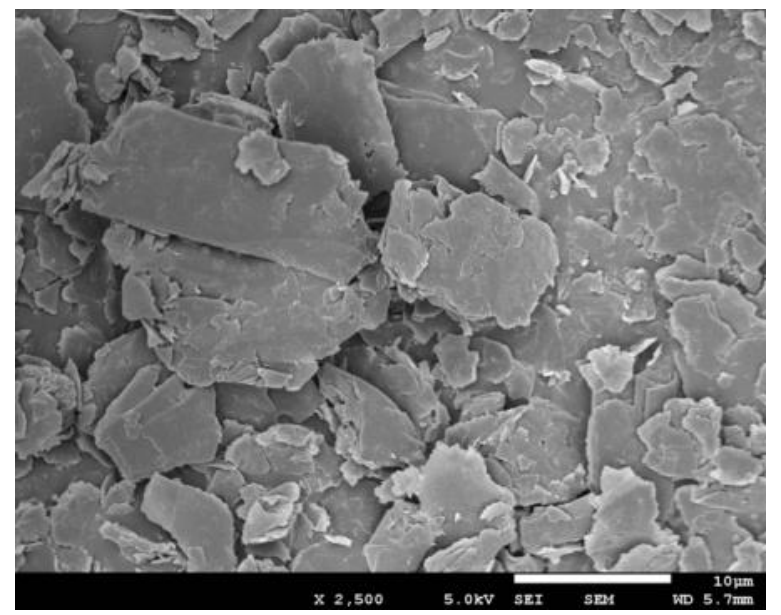

Figure 1. SEM micrograph of carbon black used as filler for piezoresistive sensors.

The sensor ink was deposited using a DEK 248 surface mount screen printer (DEK International, Weymouth, Dorset, UK). Each specimen was printed with a diameter of $1 \mathrm{~cm}$, using a 325 mesh screen (UTZ TECHNOLOGIES, Little Falls, NJ). Aluminum foil substrates were prepared by soaking in $1 \mathrm{M}$ nitric acid solution for 5 minutes, rinsing in DMF, and then soaking in a second DMF bath for 5 min. After drying in an oven at $80{ }^{\circ} \mathrm{C}$, the samples were immediately printed onto the substrates. In this way, the processing oils and surface oxides were removed and the surface chemistry of the aluminum substrate was modified to promote both mechanical and chemical adhesion of the composite sensors.

The mechanical testing was performed on samples using a TA Instruments Q800 dynamic mechanical analysis (DMA) instrument. Both the static and active clamp surfaces were prepared with copper foil, providing electrical contacts for the sensor surface. A 1/8 inch thick weather-resistant silicone compliance layer (McMaster Carr, \#8632K44) was used in series with the active clamp of the DMA to ensure full contact and homogeneous load distribution across the sensor face. The contact area for load application had a diameter of $8 \mathrm{~mm}$. The full thermomechanical loading setup is shown in figure 2. The room temperature characterization of the sensor was performed using a quasi-static loading scheme with a rate of $2 \mathrm{kPa} / \mathrm{sec}$ to a final load of $350 \mathrm{kPa}$. For each composition, a total of 5 specimens were tested, having 5 test runs per specimen, resulting in a total of 25 test runs. 


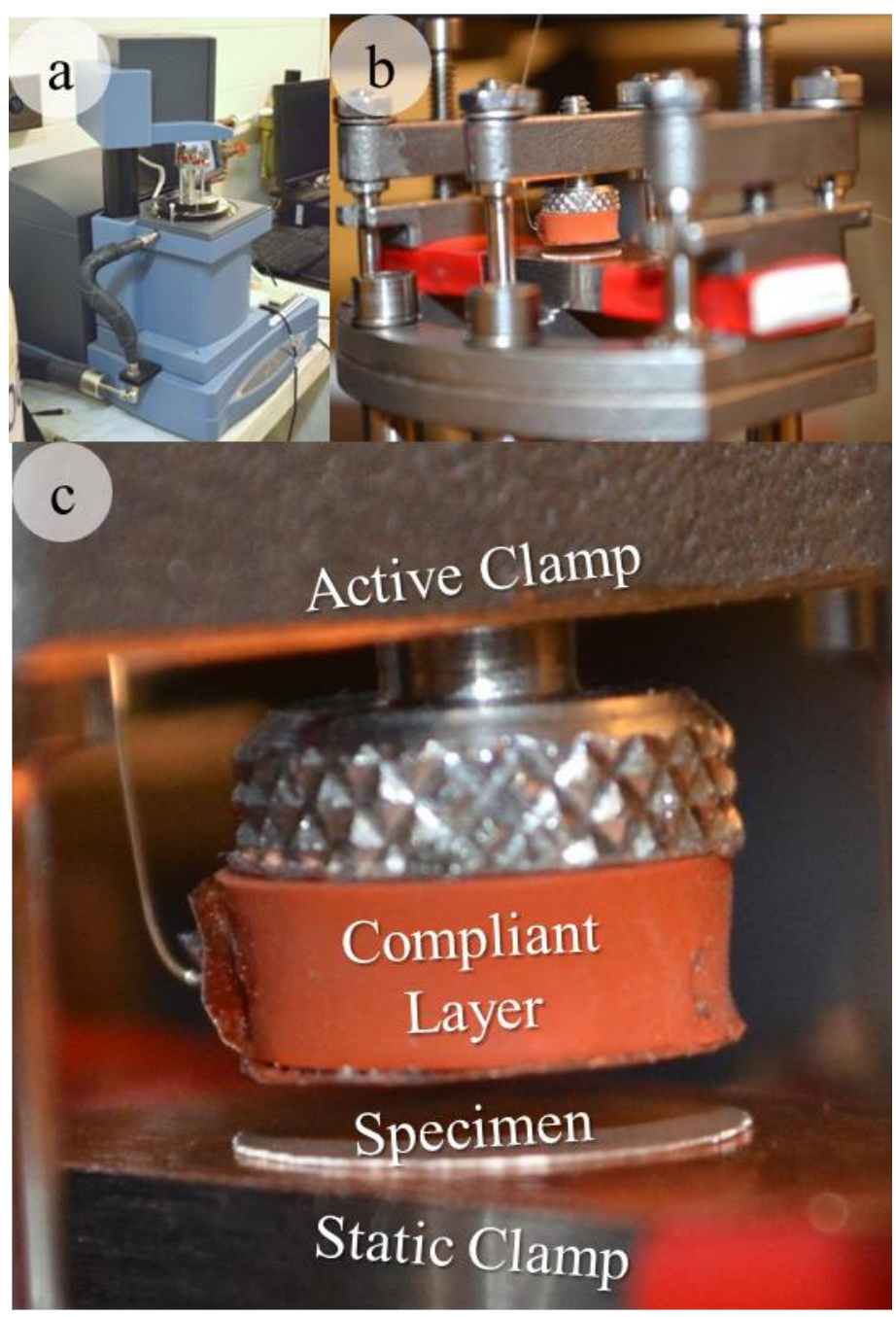

Figure 2. Thermomechanical testing setup: a) DMA instrument, b) grip configuration for testing with in situ electrical acquisition, c) compliant layer used to ensure homogenous pressure distribution.

The electrical response was recorded with a NI USB-6210 16-bit DAQ system, using the provided $5 \mathrm{~V}$ power source. In an effort to optimize the resolution of sensor response with this system, a circuit was fabricated to maximize $\Delta \mathrm{V}$ during mechanical loading using a reference resistor of $3.89 \mathrm{k} \Omega$. This circuit is depicted in figure 3 , and the sensor resistance is calculated using the equation:

$$
R_{\text {sensor }}=\frac{5 R_{\text {ref }}}{V_{o}}-R_{\text {ref }}
$$




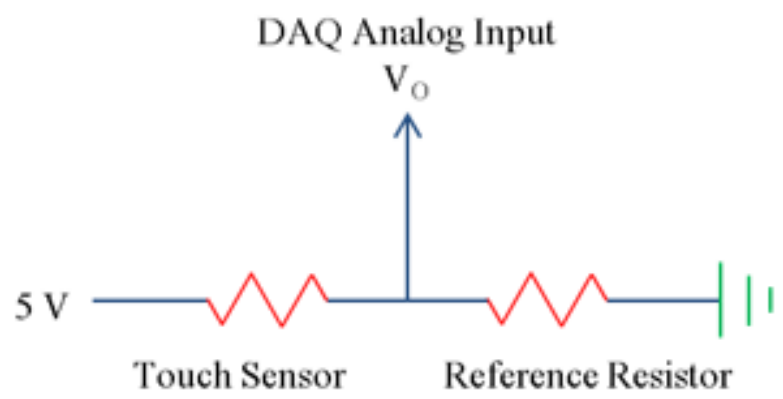

Figure 3. Circuit used to optimize sensor feedback resolution.

The industrially manufactured A201 FlexiForce (Tekscan, Boston, MA) sensors were purchased fully fabricated as shown in figure 4 , and were used for thermal characterization of piezoresistive technology. Testing was performed as previously described, and was repeated for temperature of $40{ }^{\circ} \mathrm{C}$, $80{ }^{\circ} \mathrm{C}$, and $120{ }^{\circ} \mathrm{C}$. These sensors were fully packaged, so electrical acquisition was performed by soldering directly to the provided electrodes rather than using the copper contacts.

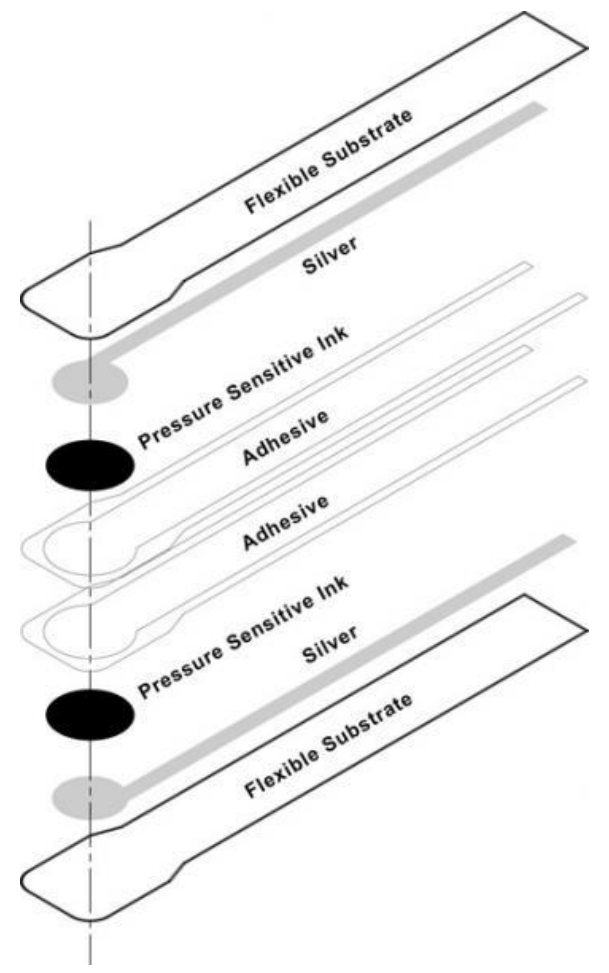

Figure 4. FlexiForce piezoresistive sensors purchased from Tekscan. 


\subsubsection{Results and Discussion}

The testing results for the fabricated piezoresistive sensors are represented by a single specimen for each filler volume loading which was testing, rather than showing results from all five sensor specimens. The room temperature sensor response (voltage) to mechanical loading (stress) is plotted for each carbon filler volume loading in figures 5-8. Notice the large amount of noise (disarray of signal) and drift (change in magnitude between runs) for each sensor. Piezoresistive results are displayed in terms of voltage rather than resistance in this work, since resistance values are non-linear making results more difficult to interpret.

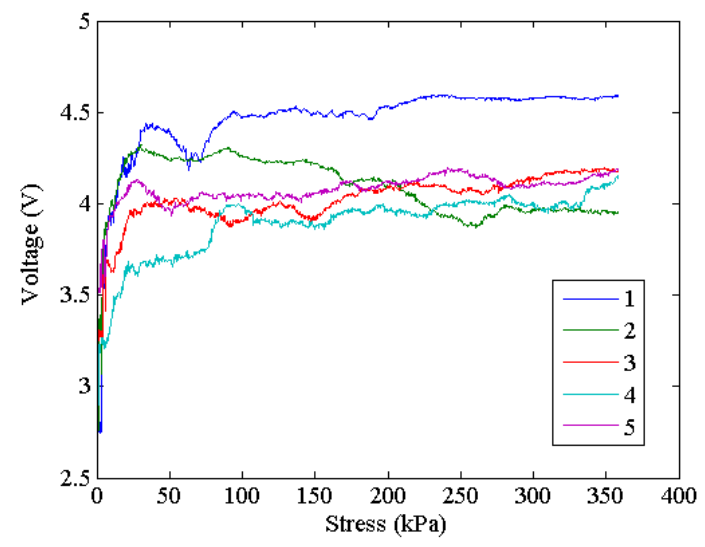

Figure 5. Response (voltage) to an applied stress for a sensor with 5 vol\% carbon filler.

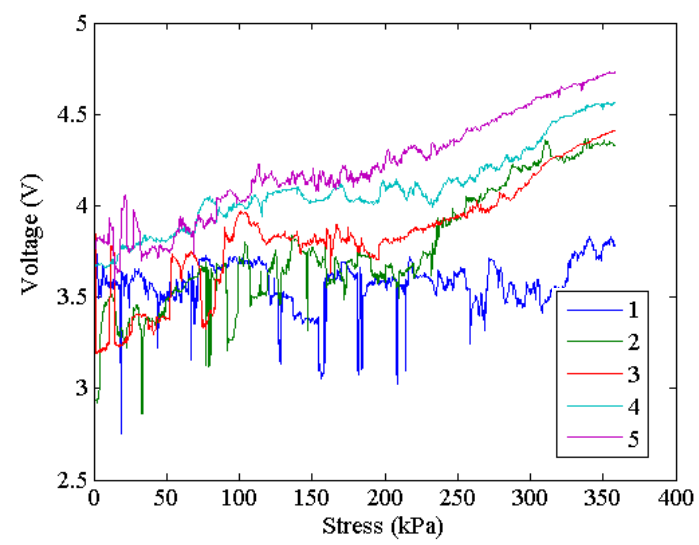

Figure 6. Response (voltage) to an applied stress for a sensor with 10 vol\% carbon filler. 


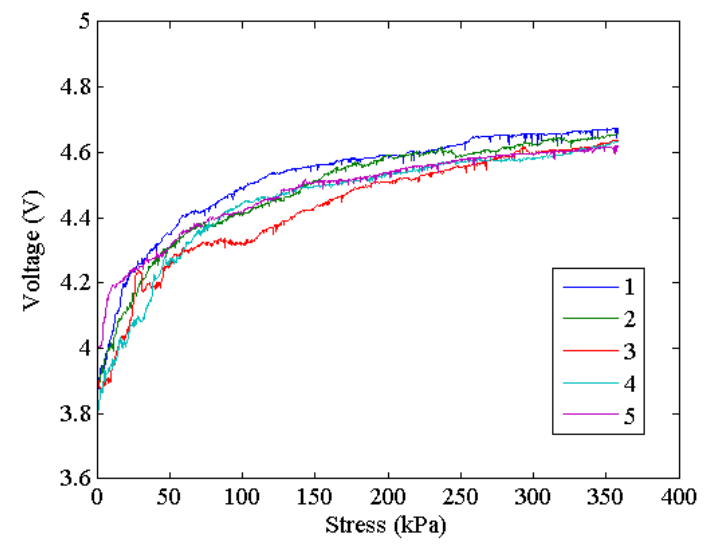

Figure 7. Response (voltage) to an applied stress for a sensor with 20 vol\% carbon filler.

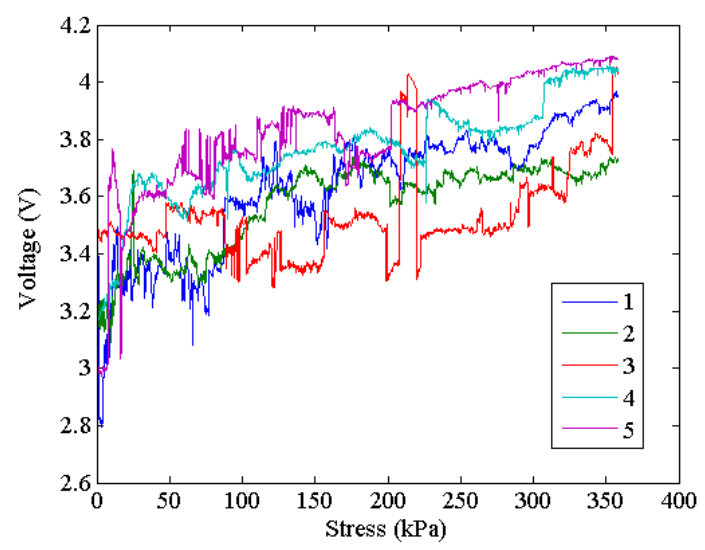

Figure 8. Response (voltage) to an applied stress for a sensor with 30 vol\% carbon filler.

There are two primary factors contributing to piezoresistivity, which are consequently the two factors which must be considered to understand sources of error. These are the resistance of a conductive path between two electrodes, and the number of conductive paths between the electrodes [57]. These paths are easily modified with applied stress/strain causing a drift in the sensor response. This is caused by re-aggregation of filler-filler bonds, and degradation of the microstructure [58-59]. These properties have long been studied, and much literature is available on these topics.

The sensor with a 5 vol\% of carbon filler had a small response, as the resistance was not significantly reduced with increased load application. Since the distance between the particles was large, the change in distance induced by strain was minimal. At the 10 vol\% filler concentration, the sensor response was increased. This was in part established by conductive paths experiencing reduced particle spacing, allowing lesser amounts of strain to more significantly influence the resistance of the bulk material. The sensor experienced large jumps with the initial loading cycles as particles likely settled in 
the microstructure, increasing gaps in the conductive path. The spikes in the response begin to fade with each additional cycle as the particles eventually come to rest. This occurs as gaps between particles in the bulk material are reduced, as alternative conductive paths are made available.

At a 20 vol\% filler loading, sufficient conductive paths are available to prevent such gaps from affecting the sensor response. However, there remains a notable drift between test cycles, and nonlinear trends begin to be introduced by the last cycle as the microstructure degrades and particles move. The sensors with a 30 vol\% filler loading experience shorting as particles become too close. This can be seen on both a large and small scale. Initial jumps in the sensor response are small, as short chains of filler materials begin to make contact. With additional loading cycles, the chains become longer and the sensor response degrades with increased shorting lengths (leakage currents).

Flexible, thick-film force sensors capable of quantifying high loads are limited in industrial availability. Flexiforce sensors, produced by Tekscan, were found to meet these requirements and were tested for their ability to withstand thermomechanical loadings schedules. A single representative sensor response is shown to summarize the results of thermomechanical testing. The sensor response (voltage) under mechanical loading up to $350 \mathrm{kPa}$ is shown in figure 9 for testing performed at temperatures of 25 ${ }^{\circ} \mathrm{C}, 40{ }^{\circ} \mathrm{C}, 80{ }^{\circ} \mathrm{C}$, and $120^{\circ} \mathrm{C}$.

The FlexiForce sensors performed excellently at room temperature, as the microstructural properties were carefully tailored to prevent excessive drift and noise. However, thermal loading of 40 ${ }^{\circ} \mathrm{C}$ introduced nonlinear characteristics, and at $80{ }^{\circ} \mathrm{C}$ additional nonlinear attributes and drift were introduced. The thermally induced nonlinearity is error, quantified by having a root-mean-square error of 0.41 at $40{ }^{\circ} \mathrm{C}$ and 0.89 at $80{ }^{\circ} \mathrm{C}$ when compared with the $25^{\circ} \mathrm{C}$ results. At $120^{\circ} \mathrm{C}$ the sensors persisted only through one or two stress cycles at best, and failed entirely under additional loading cycles.

Advancements in piezoresistive technology are feasible pending advancements in microstructural properties, the incorporation of new polymers and filler materials to reduce microstructure movement and degradation, and thermal effects caused by thermal prosperities of individual constitutes [60]. However, these microstructural challenges are extensive as compared with capacitive sensor technology. As a result, this work will not use piezoresistive sensors for harsh environment applications. Meanwhile, the piezoresistive sensors will be used for evaluating sensor packing, since they are simple to manufacture, and data acquisition is more easily performed. 

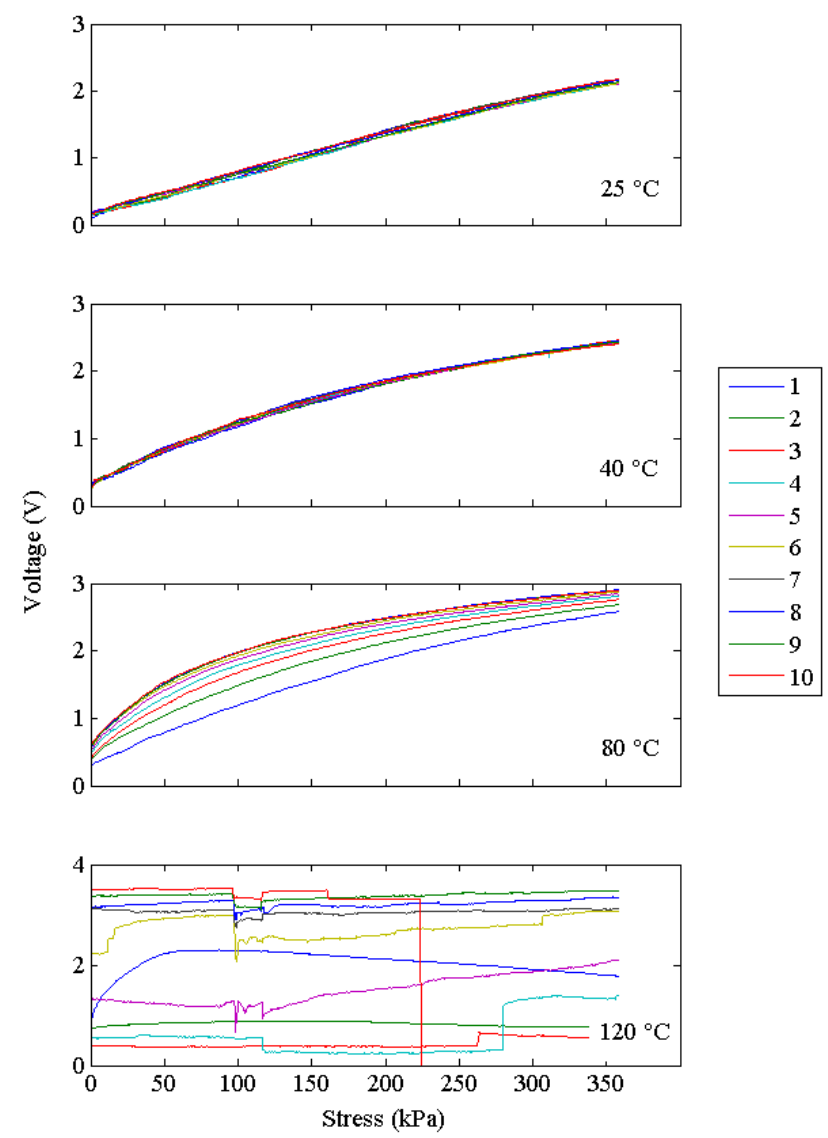

Figure 9. FlexiForce sensor response (voltage) under a ramp load up to $350 \mathrm{kPa}$ for temperatures of $25^{\circ} \mathrm{C}, 40^{\circ} \mathrm{C}, 80^{\circ} \mathrm{C}$, and $120^{\circ} \mathrm{C}$.

\subsection{Sensor packaging}

\subsubsection{Development of electrode patterns}

The first major objective in sensor packaging is the design and fabrication of a durable, flexible, thin-film, patterned electrode. In an effort to achieve this goal, two primary approaches were taken: 1) screenprinting and drying of an ink to form a thick polymer/Ag film, and 2) DC sputtering of an Ag thin film. Conductive inks were fabricated using the same binder matrix as the piezoresistive inks (PVDF, BPA, DEGBE), but using a high volume content of silver micro-powder as the filler (85-95 vol\%). Conductive inks were screen printed onto Teflon ${ }^{\circledR}$ films, which were mechanically treated by the manufacturer to promote physical adhesion of the inks, and untreated Kapton ${ }^{\circledR}$ polyimide films. 
The electrode's flexure durability was tested by evaluating increased resistance as a function of crease folding. Kapton ${ }^{\circledR}$ and Teflon ${ }^{\circledR}$ films were prepared with printed electrodes, and resistance was measured with a digital multimeter (DMM) before and after continued crease folding repetitions. Crease folds were applied by simply folding the film back upon itself, and applying approximately $1 \mathrm{lbf}$ to the fold by hand. While the electrodes exhibited great conductivity under high radius flex, they were found to experience cracking during the crease folding which ultimately resulted in a loss of conductivity. The ink was modified using menhaden fish oil (Z-3, Tape Casting Warehouse, Marrisville, PA) as a surfactant to disperse the silver filler. Additional organics were also implemented during fabrication of the polymer binder to promote flexibility of the electrodes, such as methacrylamide (MAM), N-N-

Methylenebisacrylamide (MBAM), and N,N,N,N Tetramethylethylenediamine (TEMED). However, the crease fold strength of the electrodes was not notably advanced. The electrodes were also found to delaminate from the film substrate during printing of the sensor composition, which shared a common solvent.

The sputtered electrodes offered superior resistance to crease folding, though the fabrication process is more extensive. The first step in photography is deposition of photoresist on the substrate. Telflon films were found to have poor wetting characteristics, and were coated with an adhesive agent, hexamethyldisilazane (HMDS) prior to being coated with the photoresist. However, the HMDS was not removed during the exposure and development process, causing the electrodes to be removed during the lift-off process.

A new method was designed for wetting the Teflon ${ }^{\circledR}$ substrates. The Teflon ${ }^{\circledR}$ substrates were coated with photoresist using a two-stage process to promote wetting. First, the films were placed on a polished wafer and AZ $5214 \mathrm{E}$ image reversal photoresist was applied while a spin coating speed of 6,600 rpm was maintained for 60 seconds. This was the minimum speed found to consistently overcome wetting resistance with centrifugal force. If the vacuum on the chuck was not maintained or if the wafer was not properly centered, silicon wafers were found to release and shatter. For this reason comparable polished aluminum wafers were used. The film was immediately removed and dried on a hot plate at 95 ${ }^{\circ} \mathrm{C}$ for one minute; higher temperatures were found to deform the Teflon ${ }^{\circledR}$ film.

Because of the high rpm, the thickness of this photoresist layer was considered to be negligible. Sequential deposition steps of the photoresist were performed, where the deposition chuck was ramped at $330 \mathrm{rpm} / \mathrm{sec}$ to $440 \mathrm{rpm}$. The $440 \mathrm{rpm}$ was maintained for 10 seconds before ramping at $440 \mathrm{rpm} / \mathrm{sec}$ to 3,000 rpm. The 3,000 rpm was maintained for 15 seconds before the spinner was turned off. The film was removed and dried on a hot plate at $95{ }^{\circ} \mathrm{C}$ for three minutes. The coated films were loaded into the Microtech MA6 mask aligner and exposed for 200 seconds. They were then developed in AZ 310 MIF 
for approximately 30 seconds. DC sputtering was performed to deposit a thin metallic layer, and an acetone lift-off process was performed to obtain the patterned electrodes.

Numerous metals and deposition thicknesses were evaluated for sputtering, testing conductivity and resistance to cracking under crease folding. Copper was found to have excellent adhesion with the Teflon ${ }^{\circledR}$ film, and aluminum experienced cracking and flaking caused by poor adhesion. Both copper and aluminum were found to form an oxide over ambient exposure, resulting in extremely low or nonconductive films. For this reason, silver and platinum were evaluated for potential use. Platinum was found to have better adhesion and resistance to cracking, though silver was comparable. Sputter thicknesses were estimated from sputter time with prior knowledge of deposition rate established using an alpha step stylus profiler (profilometer), revealing a narrow optimal thickness in the range of 0.5 to $1 \mu \mathrm{m}$. Films thinner than $0.5 \mu \mathrm{m}$ lacked interconnectivity, resulting in poor conductivity. Alternatively, films thicker than $1 \mu \mathrm{m}$ became dense and bulky, experiencing a brittle nature with cracking.

\subsubsection{Design of layered packaging techniques}

Full packaging of the sensor is required to protect the array from electrical shorting and mechanical abrasion. In this section, all of the proposed packaging techniques included a 5 layer design. Shown in figure 10, this design uses power and ground electrodes sputtered onto the polymer substrates, and a sensor material is printed onto the powered electrode. It is important to note that after adhesion of the two polymer substrates between electrodes, the sensors sensor rests in an open circuit configuration until minimal pressure is applied to promote contact between the electrodes. 


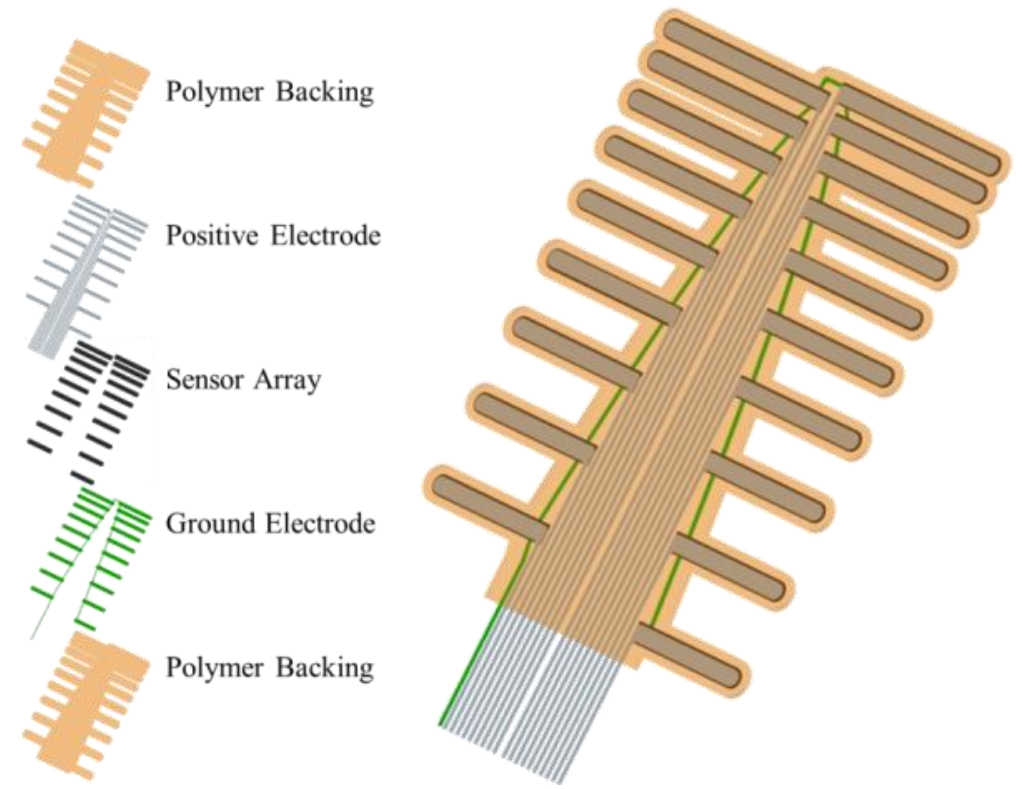

Figure 10. Sensor package design, showing protective polymer films and electrode patterns.

Adhesion of the polymer substrates was carried out using a variety of methods. A double sided tape was adhered to Mylar film and shaped with a laser cutter, then transferred to the polymer backings. Use of tape was widely successful and simple to implement, though even thin layers of tape notably increased the distance between electrodes which causes the sensor to rest in open circuit. Gluing the substrates was more difficult and results varied, though it reduced the gap size which causes an open circuit before loading of the sensor. This is the method used in industry for flexible film piezoresistive sensors.

The most successful gluing method was accomplished by cutting a thick-film with a laser cutter, creating a shadow-mask. The mask was then laid over the sensor backing and an aerosol based adhesive was sprayed, being deposited on the backing where the shadow-mask did not cover. Various sensor packages fabricated with different adhesion methods are shown in figure 11. Neither the use of a heat press or plastic welding were successful, as both caused small deformation in the polymer film which caused cracking in the electrodes. Cracking of the electrodes caused by thermal treatment is shown in figure 12 . 

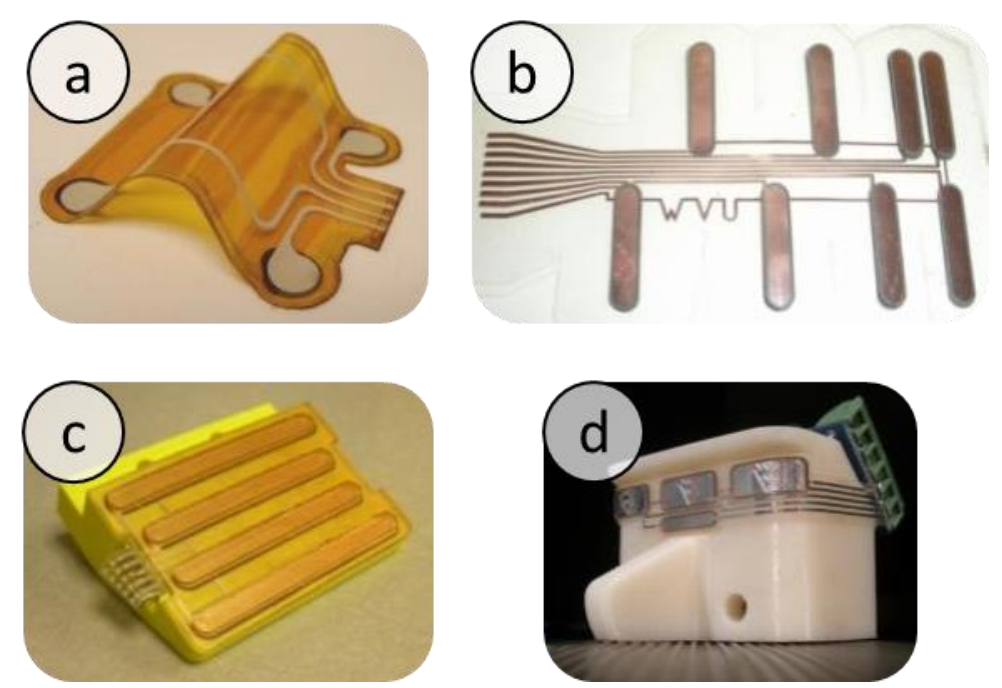

Figure 11. Thin film piezoresistive sensors adhered by a) paper based tape, b) aerosol based glue, c-d) polymer based tape.

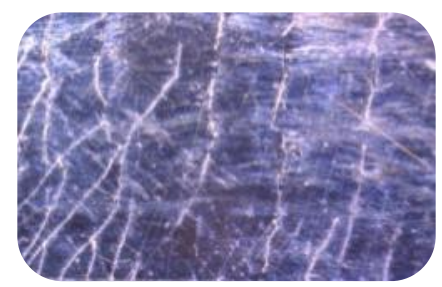

Figure 12. Electrode cracking caused by deformation of the polymer.

Packaged sensors were embedded in a rubber matrix in an effort to demonstrate their potential use in composite material designs. The polymer substrates were perforated to allow for interconnection of the matrix, assisting with the reduction of point concentrations caused by implementation of a foreign object in the composite laminate structure. The embedded sensors were not tested, but were used to show embedding potential for future applications. Embedding was performed by pouring the desired matrix material into a mold prepared with a generic release agent (figure 13), placing the trimmed and perforated sensor array onto the mold, and then pouring additional matrix material over the back of the sensor array. The aluminum mold was agitated vigorously by hand to assist with removal of bubbles in the matrix. Figure 14 shows one such embedded sensor array, where small hillocks were implemented to encourage contact at the location of the sensors, and to assist with dispersing point loads across the sensor face. Using a two-part conductive silver epoxy diluted by approximately 30 vol\% acetone, $130 \mu \mathrm{m}$ silver wire was adhered to electrodes. 


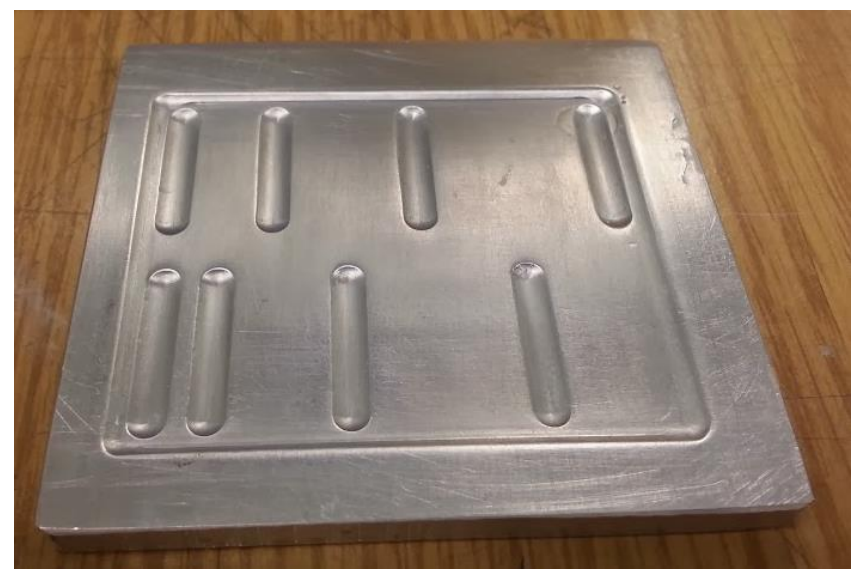

Figure 13. Aluminum mold for casting an embedded sensor prototype with hillocks to ensure first contact on sensor face.

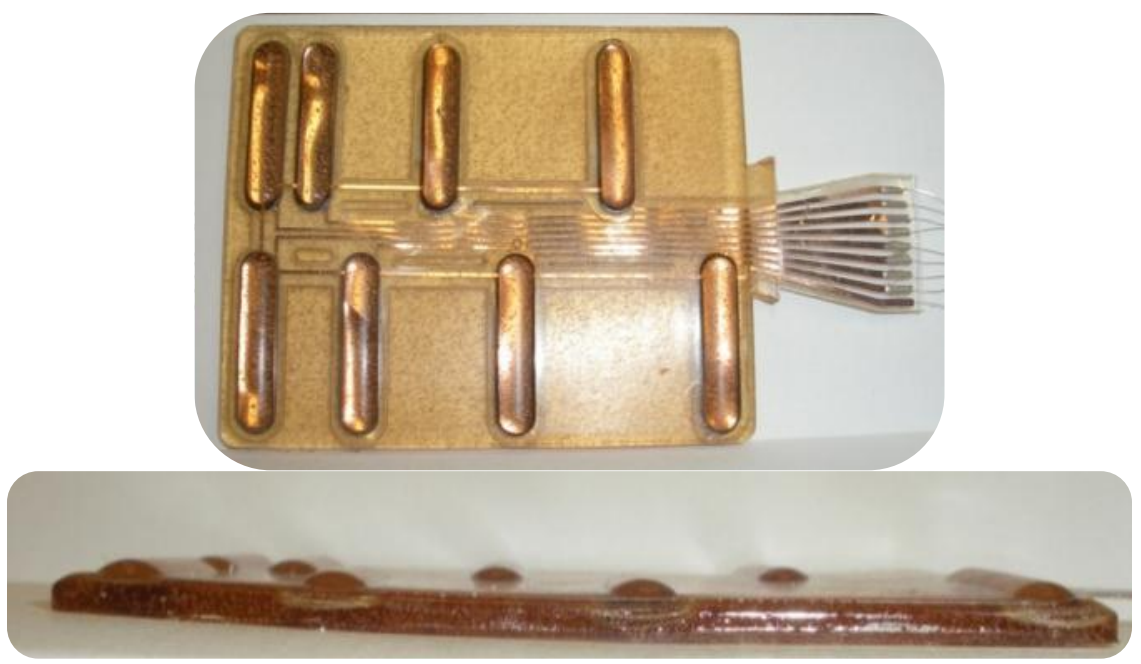

Figure 14. Embedded sensor prototype, showing a thick-film sensor embedded within a rubber matrix.

These simple piezoresistive prototypes function well for quick production, custom, disposable sensor arrays for lab testing. When prepared with an adhesive backing, the sensors can be quickly be implemented into existing robotic grippers with no modifications or damage to the grippers. When the sensors are combined with an RJ-11 jack and a graphic user interface (figure 15), these sensor packages have been integrated as a plug-and-play solution for force and spatial awareness for robotic demonstrations. 


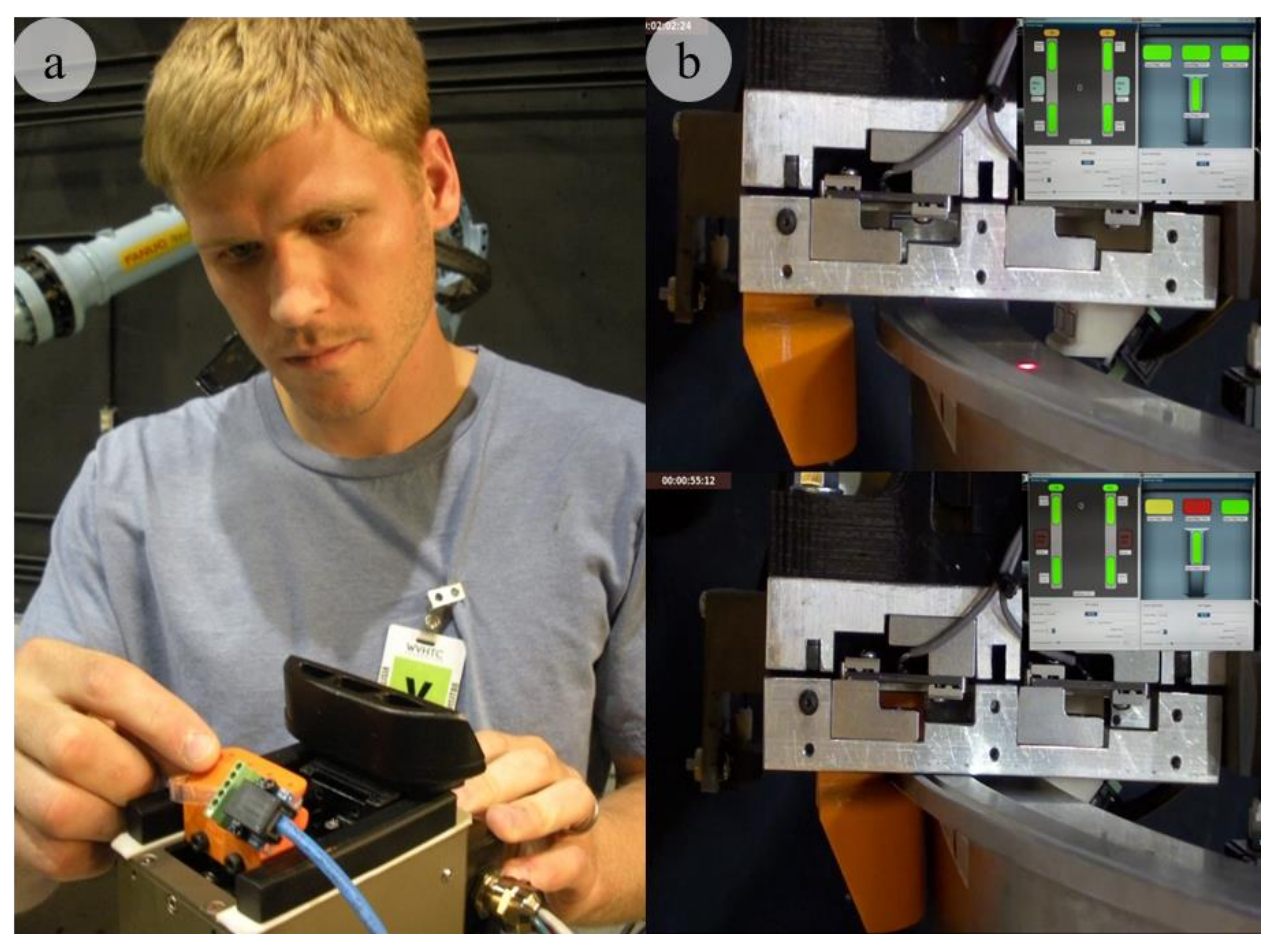

Figure 15. Demonstration of plug-and-play sensor use: a) applying sensor and connecting to DAQ, b) sensors used for real time contact information with graphic user interface.

The processing design shown in figure 10 was found to produce sensors that occasionally open circuit, since the electrode layers are not intimately bonded to the sensor composition. This same issue was found with the industrially available FlexiForce sensors. In order to further improve the design, new processing procedures were proposed. As shown in figure 16, the same five layers are proposed differing only in deposition technique of the electrode and insulating layer added after the sensor composition. After depositing the electrode pattern and sensor composition onto the Kapton $®$ film, the platinum ground electrodes are added using photolithography processes. Finally, a top insulating layer composed of silicone is deposited by spin coating. The final package is trimmed using a laser cutting system. Use of Kapton ${ }^{\circledR}$ film, controlled volatility (designed to meet outgassing standards) silicone, and platinum provided a sensor package able to resist thermal and outgassing effects. The package demonstrated in figure 16 has total thickness of $50 \mu \mathrm{m}$. 


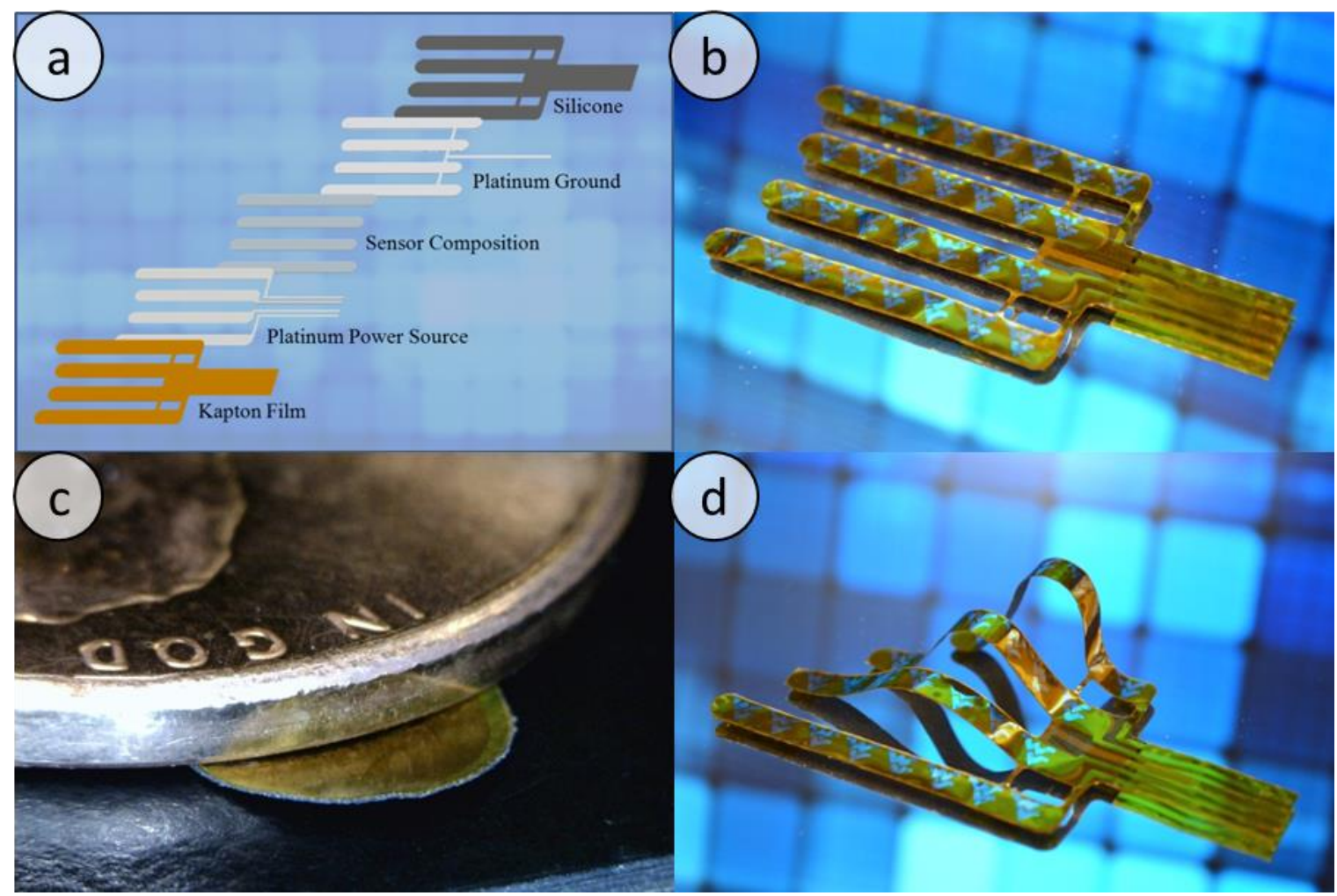

Figure 16. Sensor package: a) deposition schedule for sensor package fabrication, b) fully fabricated sensor package, c) thickness as compared with a penny, d) flexibility of sensor package.

The use of a metal foil substrate was proposed to replace the Kapton ${ }^{\circledR}$ film. In this design the metal foil was used as the power electrode, further simplifying the design previously proposed. By using a material such as aluminum, the outer surface of the foil could be oxidized to provide an electrically insulating barrier. This design would provide a barrier to protect from radiation and electron bombardment. However, initial testing using a variety of polymers revealed that a large rate dependent hysteresis was being introduced into the sensor response. This can be seen in figure 17, where the sensor response (capacitance) and applied load are plotted against time. The time delay between the stimulation (force) and response (capacitance) is the result of the rate dependent hysteresis. Work in literature has shown that the elastic modulus, internal stress, and strain vary along the transverse axis of the polymer film when it was rigidly bound to a metal substrate [61]. It is believed that these attributes introduce the observed viscoelastic behavior. 


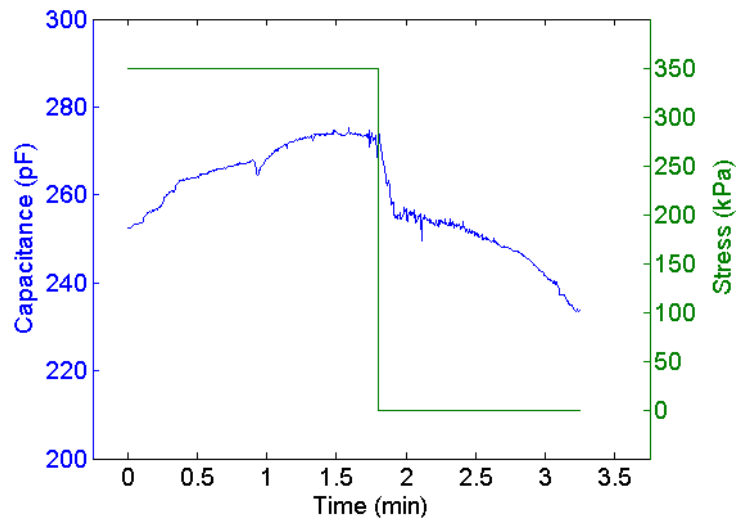

Figure 17. A mechanical stimulation (force) applied to show the sensor response (capacitance) both as a function of time. The delay between stimulation and response reveals a rate dependent hysteresis.

Alternative processing methods were developed in this work to prevent this anomaly. A release agent was prepared by adding $10 \mathrm{wt} \%$ of polyvinyl alcohol (PVA, Celvol 502, 15,000 g/mol) to DI water and the solution was then stirred at $80{ }^{\circ} \mathrm{C}$ for $20 \mathrm{~min}$. Glass substrates were hand dipped into the solution, and immediately placed in a vacuum oven at $80{ }^{\circ} \mathrm{C}$ and $15 \mathrm{in} \mathrm{Hg}$ for $3 \mathrm{hrs}$. The composite sensor material based on most solvents besides water and ethanol can then be deposited. Since polymers that adsorb water are undesirable for harsh environment applications, this design allows for casting of nearly any desired material without wetting of the PVA release layer.

After casting and curing of the sensor material into a film, the glass substrate is submerged in water and sonicated using a AMSCO Reliance Sonic 150 sonication bath at $69{ }^{\circ} \mathrm{C}$. If the edges or corners of the film adhere to the substrate they are trimmed, allowing the film to release. If a thick film was fabricated, it was simply removed by hand. In the case that a thin film is desired, the film was carefully lifted until the surface tension of the water suspended it perfectly across the top of the water. As shown in figure 18, a piece of Mylar film was then carefully placed against one edge of the film, and dipped into the water to retrieve the film. Using a laser cutter, the film was trimmed and shaped for the desired application. Finally, the film was placed on the metal foil or other substrate. Using this procedure, rigid bonding with the metal foil substrates was prevented, eliminating the observed rate dependent hysteresis. 


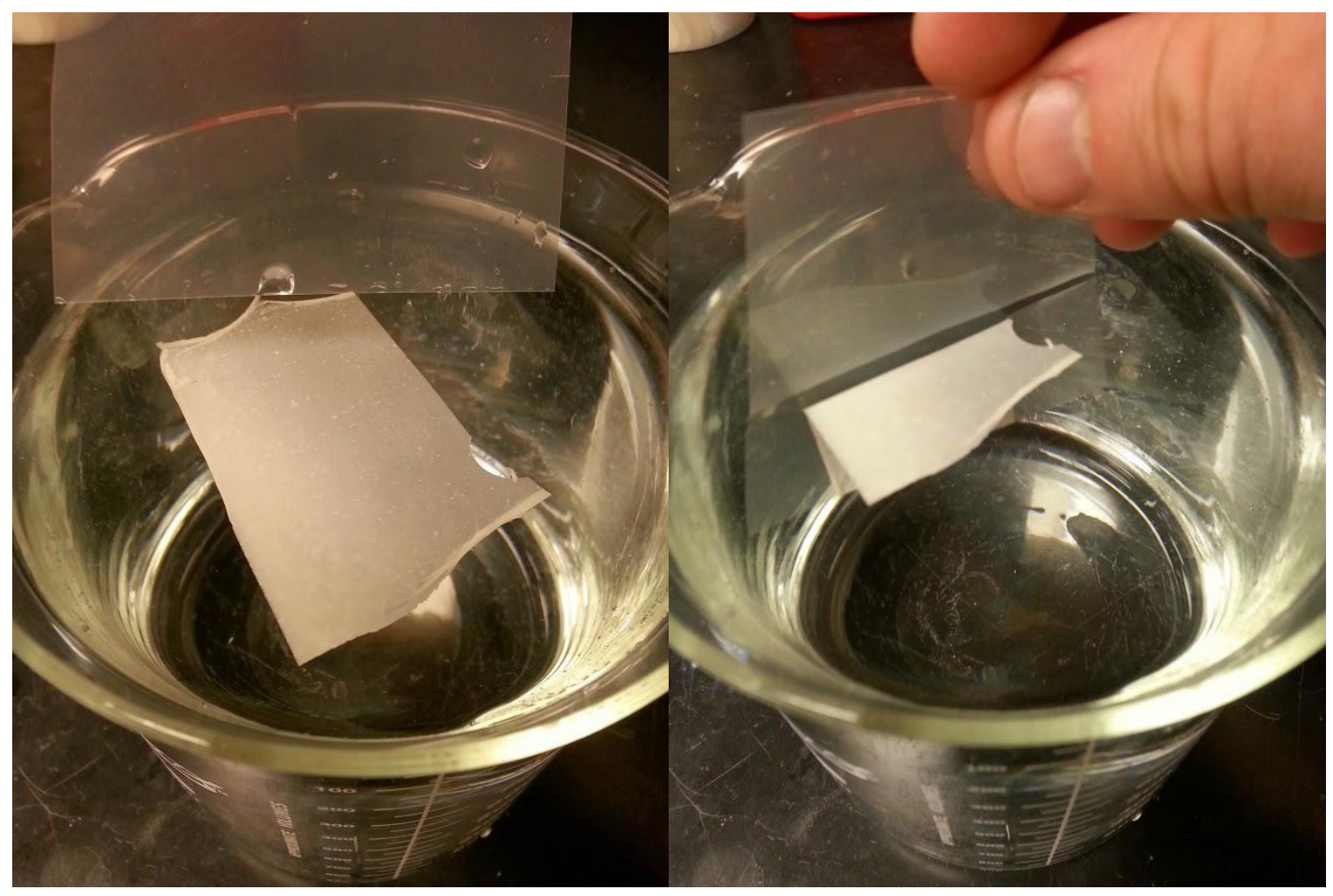

Figure 18. Thin film sensor composition being retrieved from a water bath after being released from the glass substrate.

\subsection{Conclusions}

In this work, the importance of the polymer-filler microstructure on piezoresistive sensor properties was briefly demonstrated. It was demonstrated experimentally that the conductive filler volume loading has a significant impact on the sensor performance, including sensor response drift and noise. It was also demonstrated that degradation of the polymer matrix heavily contributes to sensor longevity. Using high performance piezoresistive sensor from industry, thermal effects on the sensor microstructure were evaluated. The results showed that thermal loading had a large impact on microstructural degradation, increasing filler mobility and ultimately destroying the sensor.

Improvements to the microstructure could be made by the substitution of more robust material counterparts, and detailed evaluation of polymer-filler interactions. However, long term use the sensor will still result in some fraction of degradation and other sensor technologies are available to avoid such drawbacks. This will be further discussed in chapter 2. Piezoresistive sensors have demonstrated the ability to act as an inexpensive means for testing of sensor packaging techniques, and preliminary lab demonstrations. Use of an adhesive backing was used for quick installation of sensor packages, and a graphic user interface was developed to give real time spatial and force information. 
Methods for preparing various sensor packages have been introduced, including the need for controlled volatility and thermally resistant polymers, and conductive thin-films not prone to oxidation. The primary design is based on a polymer film substrate, depositing electrodes and sensor materials layerby-layer. The ability to embed sensor packages within a composite has also been briefly demonstrated. New processing strategies have been established to prevent the introduction of a rate dependent hysteresis in sensors deposited on metal foil substrates. Use of metal foils as a substrate is proposed to reduce the effects of radiation and electron bombardment. 


\section{Chapter 3. Experimental Design of a Ceramic-Polymer Capacitive Force/Spatial Awareness Sensor Composition.}

\subsection{Introduction}

In order to meet the demands of embedded, harsh environment applications, a number of environmental, mechanical, electrical, and volumetric restraints must be considered. For example, the environmental conditions of space include pressures lower than $10^{-7}$ torr and temperatures ranging from $80{ }^{\circ} \mathrm{C}$ to $120{ }^{\circ} \mathrm{C}$ (geostationary orbit). The space environment also includes additional complications due to electron/ion bombardment and radiation [62-63]. In order to meet the contact load requirements, sensors must withstand and measure pressures up to $350 \mathrm{kPa}$ in both static and dynamic modes, and potentially endure direct abrasion with metal contacts, in addition to the other energy bombardment issues described.

Thick film force sensors are the most commonly fabricated architectures using resistive, piezoelectric, and capacitive activities for sensing. Resistive sensors include strain gauges, piezoresistive polymers and elastomers, and quantum tunneling composites. These sensors have commonly shown drift, hysteresis, creep, and a loss in sensitivity making them unsuitable for applications requiring accurate force quantification throughout the lifetime of the sensor [64-65]. In addition to requiring charge amplifiers, piezoelectric sensors have a known drift in the sensor output, requiring additional circuitry when used in static applications. This additional circuitry requires additional power and volume for functionality, which limits their potential use in confined locations [66] [67].

This work proposes the use of capacitive sensor technology for the fabrication of force sensor arrays since they exhibit high sensitivity, low drift, reduced temperature dependence, and require low power consumption compared with piezoresistive sensors [68-69]. The functionality of these sensors is based upon a change in capacitance as a function of strain which reduces the thickness of the material, as described by the following relation:

$$
\Delta C=\frac{\varepsilon_{r} \varepsilon_{0} A}{d_{1}(1+1 / \epsilon)}
$$

In this equation, $\Delta C$ is the change in capacitance (sensor response), $\varepsilon_{r}$ is the relative permittivity (dielectric constant), $\varepsilon_{0}$ is the permittivity of free space, $A$ is the area, and $d_{l}$ is the initial thickness, and $\epsilon$ is strain. Capacitive force sensors function by characterizing the relationship between an external 
pressure and a change in capacitance caused by the introduction of strain $(\Delta d)$. This relationship can either be experimentally modeled or predicted mathematically using the compressive modulus of the sensor.

Historically, capacitive sensors were fabricated using air as the dielectric material between two electrodes. In an effort to simplify fabrication of the sensors, a thick film design was implemented which allows for simple printing of the electrodes [67-69]. Additional improvements were made by introducing high dielectric ceramics into the sensor films in order to enhance the capacitance, and thus the sensitivity for small sensor feature sizes [70]. Ceramics are the most prevalently used material in the fabrication of capacitors because of their high dielectric constant. Unfortunately, ceramic materials are not conducive for use in flexible, thick film capacitive sensors due to their high elastic modulus preventing high strain levels. The alternative use of ceramic-polymer composites instead of pure ceramics provides a means for attaining a high dielectric constant while modifying the mechanical compliance of the material for use in capacitive sensor applications. Such a composite should consist of isolated micro/nano-ceramic particles within a polymer matrix to ensure consistent and homogenous mechanical and electrical properties. This design was pioneered by Newnham et al., where connectivity of the microstructure was termed as a 0:3 composite. This notation denotes a filler phase with no interconnectivity in all three dimensions (0interconnectivity) within a matrix exhibiting three-dimensional interconnectivity (3-interconnectivity) [71].

Most flexible force sensor technologies originated in the biomedical research area and lack the design criteria for use in more extreme temperature environments and cycling conditions. Therefore, many of these sensor platforms are not suitable for applications such as the on-orbit space servicing application previously discussed $[68,70]$. The aim of this work is to initiate research on flexible, capacitive force sensors based on a 0:3 composite design for use in harsh environments, specifically aiming the architecture towards a film that may be embedded below a thin metal skin on current space servicing robotic end effector designs. The thickness criteria for these sensors is defined by the need to embed the active sensor material (with patterned interconnect leads) within the end effector in order to protect the sensor from abrasion, and energy bombardment.

Selection of the active composite materials (ceramic dielectric and organic binder) was purposely chosen to represent a classification of materials that would be representative of those that would be demeaned space qualified. These material sets, and the methods and architecture used for the materials, act as a standard baseline for the future use of space qualified materials in future work. Furthermore, the scope of this work included the design and implementation of characterization and testing procedures necessary for qualifying electromechanical and thermomechanical responses of the sensor materials in the thermal conditions defined by in-orbit robotic operations. Evaluation criteria included the size constraints 
(for ease of embedding in existing metal parts), electrical, mechanical, and thermal properties affected by the material phase transitions and microstructure variables.

There are a number of variables to be considered in optimizing sensor properties, including sensor thickness, ceramic composition/particle size/volume content, the molecular weight and molecular architecture of polymer and modifier components, and the polymer/modifier ratio. A full two level design of experiments for evaluating all seven variables would require $2^{7}$ samples, which considering 5 specimens per sample results in 640 sensors to be characterized. In an effort to maximize efficiency, allowing for an increased number of experimental characterization procedures being performed on each specimen, use of a Taguchi experimental design matrix was implemented. The Taguchi method works by using combinations of results to statistically approximate the influence of a single variable. This is explained in more detail in section 3.2.1. From this experimental design, an 8 sample matrix (40 specimens) was required to be tested for each characterization method.

\subsection{Experimental}

\subsubsection{Taguchi Design of Experiments}

The Taguchi method was used to design and evaluate the experiments within this work in order to quantify the effects previously described on the sensor performance. The design includes seven unique variables, each having two layers as shown in Table 1. This design is referred to as a $2^{7}$ matrix (or L8), since it requires eight different compositions to evaluate seven variables, with two layers each. While the two layer design falsely assumes linear trends for all variables considered, it significantly reduces the number of sensor compositions required to complete the testing matrix. This design was strategically selected to decrease testing time, allowing for basic trends to be evaluated for a large number of different testing procedures. The influence of a single variable is approximated by taking the average of all sensors with each level of the variable being considered. For example, the effect of the sensor thickness is found by considered the average properties of sensors A-D, and that of sensors E-H. 
Table 1. Taguchi Design of Experiments.

\begin{tabular}{|c|c|c|c|c|c|c|c|}
\hline Sensor & $\begin{array}{l}\text { Thickness } \\
150 / 300 \mu \mathrm{m}\end{array}$ & $\begin{array}{c}\text { Dielectric Material } \\
\mathrm{PZT} / \mathrm{BaTiO}_{3}\end{array}$ & $\begin{array}{l}\text { Particle Size } \\
100 \mathrm{~nm} / 1 \mu \mathrm{m}\end{array}$ & $\begin{array}{c}\% \text { Dielectric Filler } \\
10 / 20 \mathrm{~V} \%\end{array}$ & $\begin{array}{c}\text { Modifier : Binder } \\
1: 8 / 1: 4\end{array}$ & $\begin{array}{c}\text { Binder } \\
\text { PVDF/PVP }^{\text {a }}\end{array}$ & $\begin{array}{c}\text { Modifier } \\
\text { DEGBE/MPTS }\end{array}$ \\
\hline A & $150 \mu \mathrm{m}$ & PZT & $100 \mathrm{~nm}$ & $10 \mathrm{~V} \%$ & $1: 8$ & PVDF & DEGBE \\
\hline B & $150 \mu \mathrm{m}$ & PZT & $100 \mathrm{~nm}$ & $20 \mathrm{~V} \%$ & $1: 4$ & $\mathrm{PVP}^{\mathrm{a}}$ & MPTS \\
\hline $\mathrm{C}$ & $150 \mu \mathrm{m}$ & $\mathrm{BaTiO}_{3}$ & $1 \mu \mathrm{m}$ & $10 \mathrm{~V} \%$ & $1: 8$ & $\mathrm{PVP}^{\mathrm{a}}$ & MPTS \\
\hline $\mathrm{D}$ & $150 \mu \mathrm{m}$ & $\mathrm{BaTiO}_{3}$ & $1 \mu \mathrm{m}$ & $20 \mathrm{~V} \%$ & $1: 4$ & PVDF & DEGBE \\
\hline E & $300 \mu \mathrm{m}$ & $\mathrm{PZT}$ & $1 \mu \mathrm{m}$ & $10 \mathrm{~V} \%$ & $1: 4$ & PVDF & MPTS \\
\hline F & $300 \mu \mathrm{m}$ & PZT & $1 \mu \mathrm{m}$ & $20 \mathrm{~V} \%$ & $1: 8$ & $\mathrm{PVP}^{\mathrm{a}}$ & DEGBE \\
\hline G & $300 \mu \mathrm{m}$ & $\mathrm{BaTiO}_{3}$ & $100 \mathrm{~nm}$ & $10 \mathrm{~V} \%$ & $1: 4$ & $\mathrm{PVP}^{\mathrm{a}}$ & DEGBE \\
\hline $\mathrm{H}$ & $300 \mu \mathrm{m}$ & $\mathrm{BaTiO}_{3}$ & $100 \mathrm{~nm}$ & $20 \mathrm{~V} \%$ & $1: 8$ & PVDF & MPTS \\
\hline
\end{tabular}

${ }^{\mathrm{a}}$ Mixture of $50 \%$ PVP and $50 \%$ PVDF

\subsubsection{Materials and Processing}

As seen in Table 1, the eight sensors were labeled $\mathrm{A}$ through $\mathrm{H}$ for ease of reference. Both the polymer binder and organic modifier materials were used off-the-shelf with no modifications, and were chosen by their differences in molecular weight and molecular architecture. Sensor materials consisted of polyvinylidene fluoride (PVDF, Alfa Aesar, 500,000 g/mol MW), polyvinylpyrrolidone (PVP, Fisher Scientific, 8,000 g/mol MW), diethylene glycol monobutyl ether (DEGBE, Alfa Aesar, 162.23 g/mol MW), and 3-Methacryloxypropyltrimethoxysilane (MPTS, Alfa Aesar, 248.35 g/mol MW). The modifiers were chosen for the differences in their molecular architectures, having simple surfactant like features and complex adhesion promoting features, modeled in figure 19.

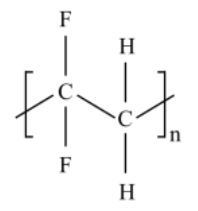

PVDF

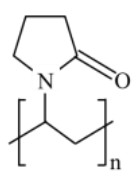

PVP

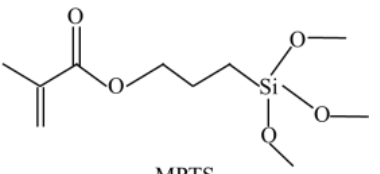

MPTS

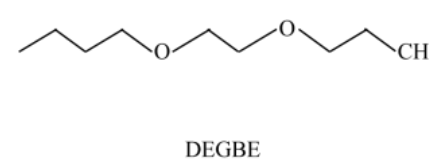

Figure 19. Polymer binder and organic modifier molecular architectures.

The ceramic $\mathrm{Pb}(\mathrm{Zr}, \mathrm{Ti}) \mathrm{O}_{3}(\mathrm{PZT})$ with a property of Navy Type II (Piezo Kinetics Inc., PKI-502) and an average particle size of $1 \mu \mathrm{m}$ was used in this work. The room temperature dielectric constant of this 
material was 2,000 at $1 \mathrm{kHz}$. In an effort to attain a second particle size of $100 \mathrm{~nm}$, a portion of this material was attrition milled with $200-400 \mu \mathrm{m}$ Ce-stabilized zirconia oxide grinding media in an ethanol solution for $10 \mathrm{~h}$. After milling, laser diffraction particle size analysis (Malvern Mastersizer 2000S) was completed, which showed that the desired particle size had been achieved. Scanning electron microscope (SEM) micrographs revealed an increased number of particles in the range of $100 \mathrm{~nm}$, but a population of $\sim 1 \mu \mathrm{m}$ particles remained after the milling process, as shown in figure $20 \mathrm{a}$. The ceramic $\mathrm{BaTiO}_{3}(\mathrm{BTO}$, Inframat, Manchester, CT) was used with a particle size of $100 \mathrm{~nm}$ and a dielectric constant in the range of 4,000 [72]. A portion of material was thermally coarsened to attain a second particle size of $1 \mu \mathrm{m}$, using a temperature of $600{ }^{\circ} \mathrm{C}$ for $2 \mathrm{~h}$ with heating and cooling rates of $3{ }^{\circ} \mathrm{C} / \mathrm{min}$. The resulting material was ball-milled with $6.25 \mathrm{~mm}$ cylindrical $\mathrm{Mg}$-doped $\mathrm{ZrO}_{2}$ media in an ethanol solution until laser diffraction particle size analysis showed that the desired particle size had been achieved. SEM micrographs showed the increased particle size to be a result of mass agglomeration rather than coarsening, with agglomerations ranging from 1 to $5 \mu \mathrm{m}$.

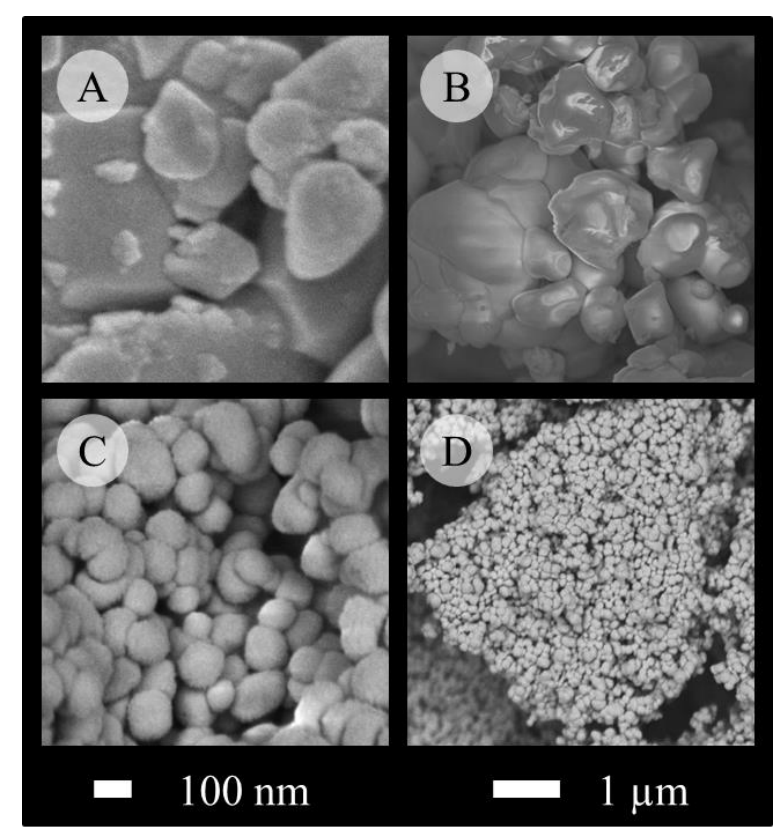

Figure 20. SEM micrographs of the ceramic particles (a) milled PZT, (b) as-received PZT, (c) as-received BTO, (d) calcined BTO.

Fabrication of the $0: 3$ connectivity composite was initiated by creating a solvent solution in a Nalgene ${ }^{\circledR}$ container, consisting of nMP and DMF with a 2:1 ratio by weight. The PZT or BTO particles were added to the solution, and dispersed by sonicating for $1 \mathrm{~min}$ at $30 \%$ power with a Sonics Vibra-cell sonication wand. The modifier material, DEGBE or MPTS, was added using a pipette. The PVDF or 
PVP polymer was weighed, then added to the solution and mixed with the unpowered sonication wand. After combining the ingredients, the solution was further sonicated for $2 \mathrm{~min}$ at $95 \%$ power. Immediately following this step, the solution was poured onto a Mylar film and cast using a micrometer adjustable tape casting blade with a $5 \mathrm{~cm}$ doctor blade. The casting thickness was set to $150 \mu \mathrm{m}$ or $300 \mu \mathrm{m}$ and a feeler gauge was used to verify the thickness. The resulting film was left at atmospheric conditions for $48 \mathrm{~h}$, and then dried in a vacuum oven at $70{ }^{\circ} \mathrm{C}$ and 15 in $\mathrm{Hg}$ for $48 \mathrm{~h}$. Compositions $\mathrm{E}$ and $\mathrm{H}$ were an exception, as they required significantly longer drying times to prevent curling of the films. Electrode films were sputtered on either side using a CVC 610 DC magnetron sputter station with a $5 \mathrm{~cm} \mathrm{Ag} \mathrm{target,}$ a vacuum of $5.0 \times 10^{-6}$ torr, and an Ar flow rate of $6 \mathrm{ccm}$, to achieve a thickness of approximately $500 \mathrm{~nm}$. Sensor specimens were created from the film using an $8 \mathrm{~mm}$ diameter punch (unless when otherwise noted).

\subsubsection{Testing Schemes}

Mechanical testing was performed on the film samples using a TA Instruments Q800 dynamic mechanical analysis (DMA) instrument. Both the static and active clamp surfaces were prepared with copper foil, providing a smooth and full surface for electrical contacts with the silver sputtered sensor faces. A weather-resistant silicone compliance layer was used in series with the active clamp of the DMA to ensure full contact and homogeneous loading. Unless otherwise noted, electrical measurements (capacitance and dielectric loss) were completed using a BK Precision 889B LCR meter at $200 \mathrm{kHz}$. The LCR leads were passed into the DMA chamber and soldered to silver micro-wires, which were attached to the copper foil creating a flexible conductor to allow in situ data acquisition during the dynamic thermo-mechanical testing scheme. The described testing arrangement is shown in figure 21, with the heating/cooling chamber door open. The testing was initiated by characterizing the room temperature response to mechanical loading. A series of nine loading steps were used as shown in figure 22. This procedure was repeated ten times for each specimen, and five specimens were used for each sensor composition (sample). 


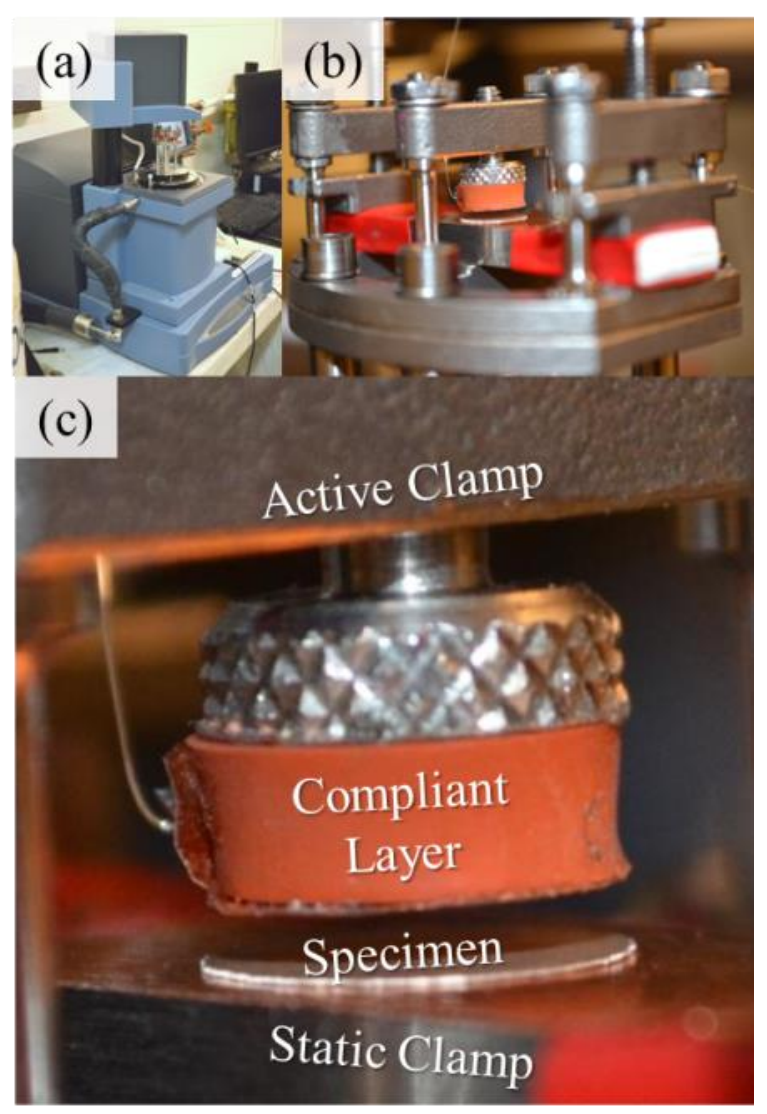

Figure 21. Thermomechanical testing setup: a) DMA instrument, b) grip configuration for testing with in situ electrical acquisition, c) compliant layer used to ensure homogenous pressure distribution.

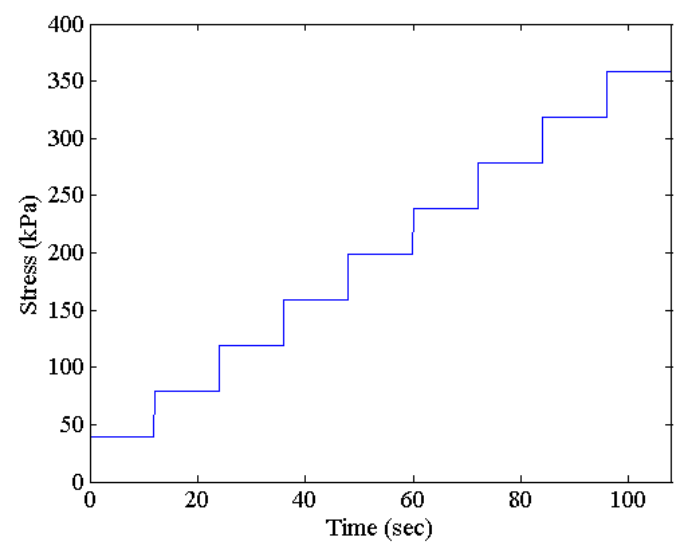

Figure 22. Applied stress versus time schedule used for the sensor response characterization.

Since the active mechanism of the sensor response is strain, the stress versus strain diagram can be used to estimate the magnitude and character of the response. The copper pads and compliance layer used for measuring the sensor response were removed to increase the accuracy of the strain 
measurements. Additionally, bare sensors were tested having no silver coating deposited onto their surface. Because of the finite thickness of the sensors, ten sensors were stacked when testing thin sensors (150 $\mu \mathrm{m}$ tape cast) and five were stacked when testing thicker sensors (300 $\mu \mathrm{m}$ tape cast). Compressive modulus testing was performed using a constant rate of stress of $120 \mathrm{kPa} / \mathrm{min}$. A total of twenty sequential runs were performed for each of the eight sets of sensors.

Thermomechanical testing was performed in two separate runs, low temperature and high temperature. Low temperature testing was performed at $-80{ }^{\circ} \mathrm{C},-40{ }^{\circ} \mathrm{C}$, and $0{ }^{\circ} \mathrm{C}$, dwelling at each temperature for five minutes before performing five loading sequences as shown in figure 23a. A load of $40 \mathrm{kPa}$ was maintained throughout the entire load to ensure contact between the leads, and the load of 350 $\mathrm{kPa}$ was used to check the sensor response. The specimen was then returned to atmospheric conditions completing the test sequence, and the testing sequence was repeated twice more. Similarly, high temperature testing was performed at $40{ }^{\circ} \mathrm{C}, 80{ }^{\circ} \mathrm{C}$, and $120^{\circ} \mathrm{C}$, dwelling for 10 minutes before performing the loading sequences (figure 23b). The specimens used for thermomechanical testing were chosen from those already used for measuring the capacitive response. This was completed to ensure that materials were preconditioned, allowing for shifting and settling of the microstructure caused by initial mechanical loading.
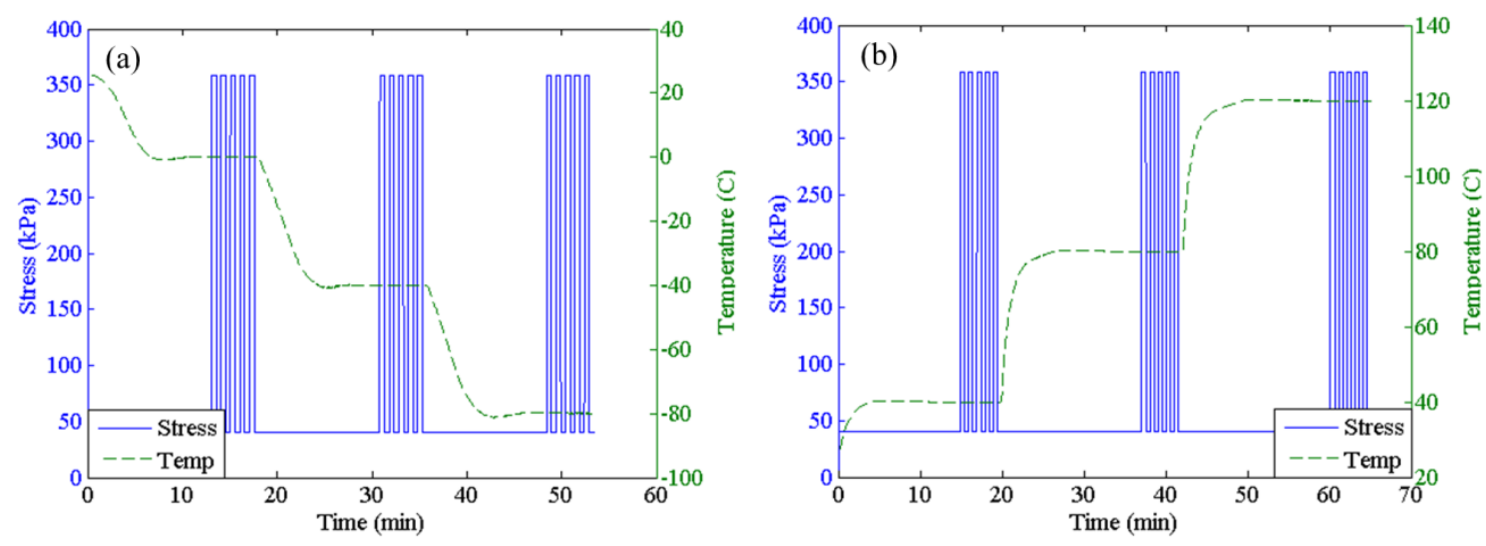

Figure 23. Applied stress versus time schedule used for thermomechanical testing at (a) low temperature and (b) high temperature.

The dielectric properties (capacitance and loss tangent) were measured as a function of temperature for the composite materials. Test specimens were increased to a $15 \mathrm{~mm}$ diameter to increase the accuracy of the electrical measurements. Testing was performed from $-80{ }^{\circ} \mathrm{C}$ to $120{ }^{\circ} \mathrm{C}$ in steps of 5 ${ }^{\circ} \mathrm{C}$ using Delta Design 9023 Environmental Chamber. Electrical measurements were taken using an HP $4284 \mathrm{LCR}$ meter with $\mathrm{AC}$ frequencies ranging from $100 \mathrm{~Hz}-1 \mathrm{MHz}$, and the data was recorded using a NI 6008 DAQ card controlled by GADD software. 


\subsection{Taguchi testing results}

The results from the room temperature response testing were used to establish baseline capacitance for the sensors. Since electrical measurements could not be made with an open circuit (since $0 \mathrm{kPa}$ load does not create a contact) the lowest applied force was used as the baseline (40 kPa load). The average of the five specimens for each composition is shown in figure 24.

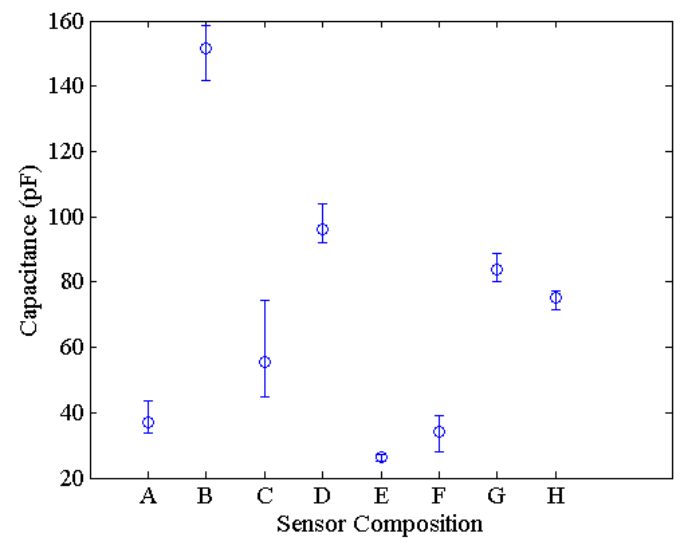

Figure 24. Room temperature capacitance of minimally loaded (40 kPa) sensor compositions at $200 \mathrm{kHz}$.

While all sensors are in a similar range, note that Sensor B showed a considerably higher capacitance. Additionally, Sensor E has a small variance while Sensor C's value was quite large. The response of the sensors is defined as the change in capacitance caused by mechanical loading, and is found by subtracting the baseline capacitance from the full capacitance value while under mechanical loading. These results are plotted in figure 25 , where the $40 \mathrm{kPa}$ measurement was found to be $0 \mathrm{pF}$ since it is considered the baseline. 


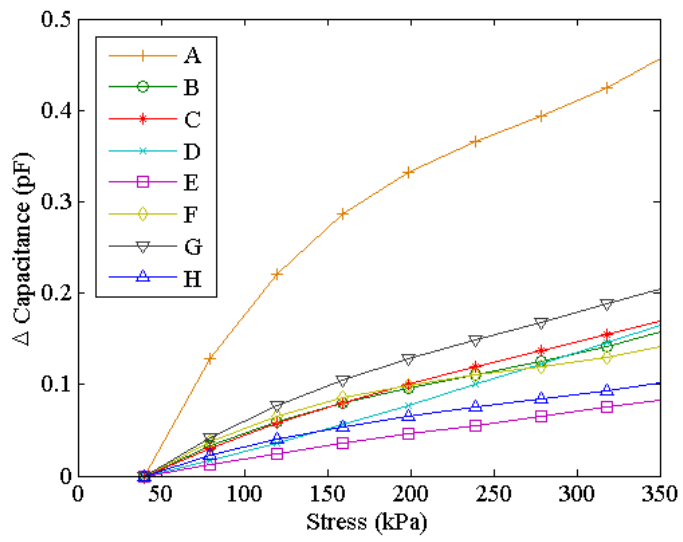

Figure 25. Room temperature capacitive response of sensor compositions at $200 \mathrm{kHz}$.

The response of Sensor A far exceeds the others, and Sensors E and H rest below the average. It was noted during processing that Sensors $\mathrm{E}$ and $\mathrm{H}$ had substantially longer drying times, and were likely denser than the other sensors. Sensor $\mathrm{H}$ was also found to have increased warping as compared with less dense films. Under closer review it was found that the average coefficient of determination converges to 0.98 for third order trend lines. This can be seen more easily with sensor A, exhibiting a linear start and a linear tail with a transition between the two linear portions, a trend requiring four points (third order).

Additional testing was performed to give insight into the non-linear effect of the ceramic content on the sensor response. Ceramic free sensors were tested as shown in figure 26. Sensor compositions are similar to Sensor A, having a 1:8 modifier / polymer ratio and using DEGBE as the modifier for all four compositions. Casting thicknesses were either $150 \mu \mathrm{m}$ or $300 \mu \mathrm{m}$, and the polymer content was either PVDF or 50\% PVDF / 50\% PVP. While the results appear to indicate a higher response without the presence of ceramics, but this would be a misinterpretation. The highest response with a purely polymer composition is found with a casting thickness of $150 \mu \mathrm{m}$ and PVDF. As demonstrated with Sensor A composition, a higher response is attainable with the addition of the ceramic material. This is further described in section 3.4.3. 


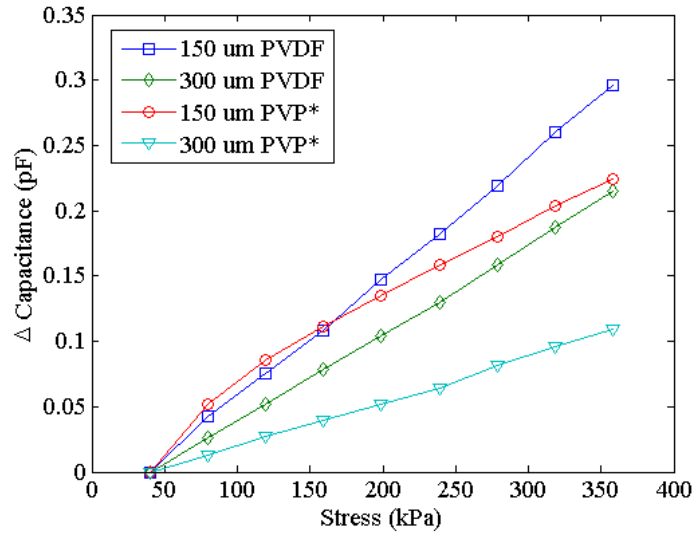

Figure 26. Room temperature capacitive response of ceramic free sensor compositions at $200 \mathrm{kHz}$.

Scanning electron microscope (SEM) micrographs show the surface morphology of each sensor composition in figure 27. The SEM shows the porous nature of the films, as well as, the large aggregates in material that do not contain PVP (Sensors A, D, E, H). The micrographs also show that the ceramic particles are dispersed homogenously throughout the microstructure, especially for Sensors G and H.

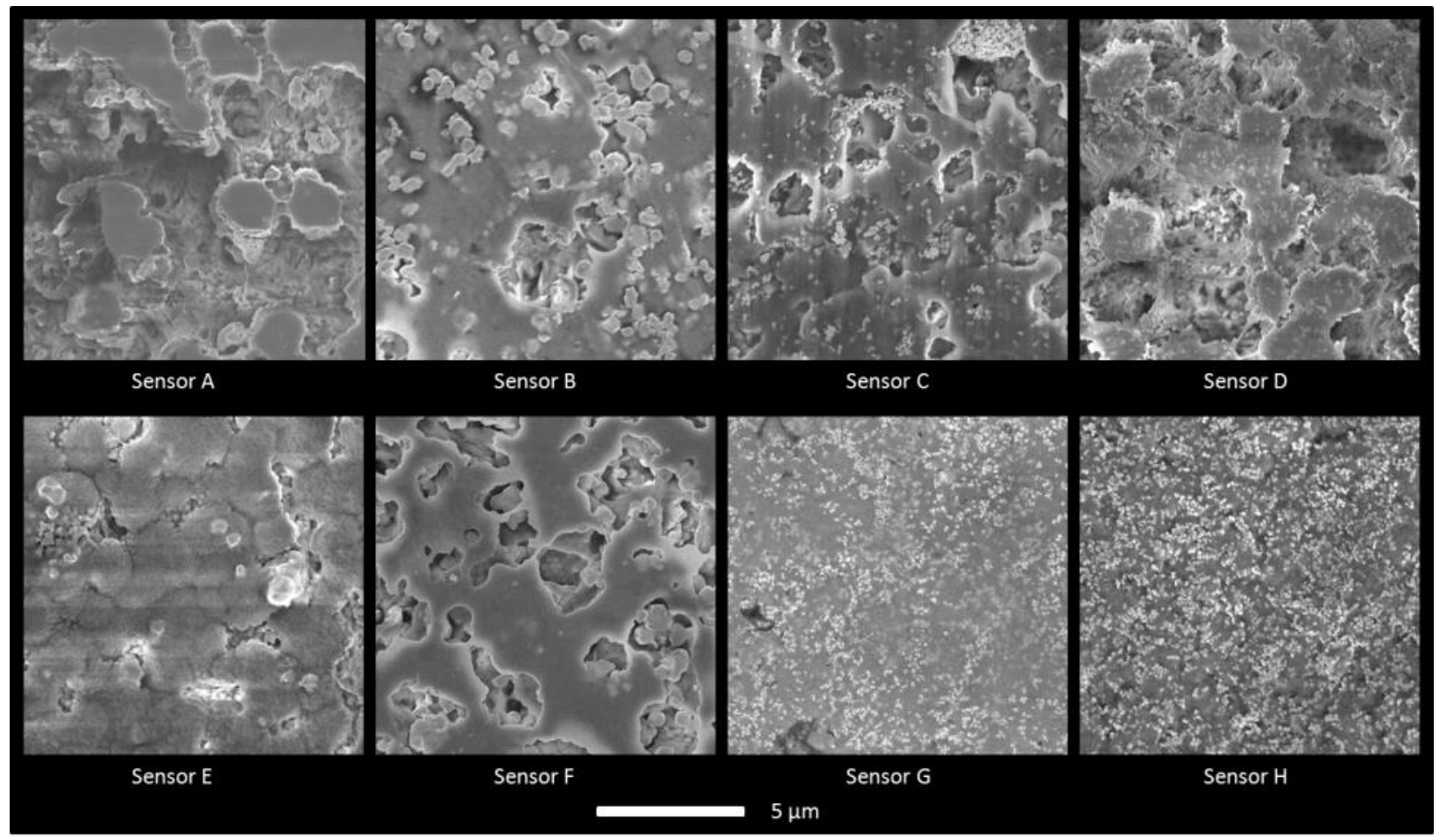

Figure 27. SEM images of the top surface of sensor materials A-H. 
The compressive modulus was calculated from the stress versus strain curves in figure 28. Sensor F experienced a compressive modulus much higher than the other sensors, and Sensor H was much lower with Sensors D and E also displaying similar trends. Analogous to the capacitive response to stress, the strain values show a third-order trend. This is not simply a similarity, as the change in capacitance is directly related to the strain. The non-linear response may be caused by the collapsing of the pore structure within the materials, in addition to the alteration in the intrinsic change in the dielectric properties described in section 3.4.7. The curves display an initial linear low modulus response and a second order transitional slope. The response ends with a high linear modulus response.

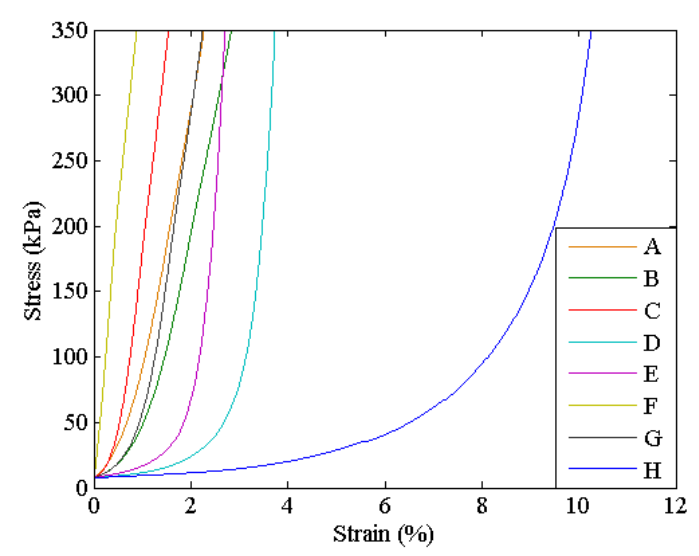

Figure 28. Stress versus strain at room temperature and stressed at a rate of $120 \mathrm{kPa} / \mathrm{min}$ for Sensors A-H.

Traditionally the modulus of a porous material is characterized by the Spriggs equation:

$$
E=E_{0}^{-b p}
$$

where $\mathrm{E}_{0}$ is the non-porous modulus, $b$ is a material constant, and $p$ is the porosity of the material. However, this equation does not efficiently convey information about the sensor properties since the porosity changes as the pores collapse with the introduction of strain. A modified version of this equation could account for such strain:

$$
E=E_{0}\left(-b\left(p_{0}-A t \varepsilon\right)\right)
$$

Within this equation, $p_{0}$ is the unloaded porosity, $A$ is the surface area of the sensor, $t$ is the original thickness of the sensor, and $\varepsilon$ is the strain introduced. This equation assumes that all strain occurs in the porous regions. While this equation more efficiently predicts the modulus of the porous materials, a more 
simplistic approach is proposed which allows for direct comparison of initial low modulus region, the transition point, and the compressed high modulus region. The low modulus of each curve was found by fitting a linear trend line from the first point to the second, third, and consecutive points until the coefficient of determination reached a value of 0.98 . The high modulus was found in a similar fashion, beginning with the last point and working consecutively backwards. These two linear slopes were then plotted, and a third line was generated to determine the intersection point of interest. The location where this third line intersects with the original curve provides the transition point, giving the stress and strain value where the material transitions from low modulus porous attributes to a higher modulus. This intersection was compared with the first and second derivatives, shown in figure 29 , to evaluate the effectiveness of the method.
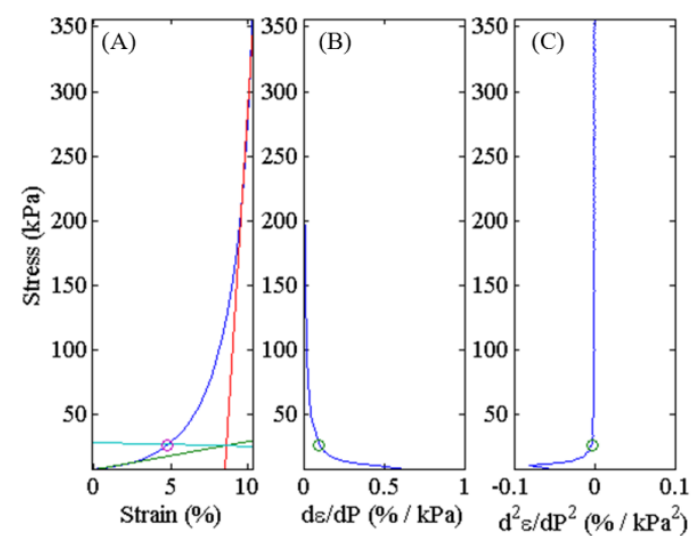

Figure 29. Applied stress versus the \% strain and derivatives for determination of the transition point between low strain and high strain deformation of Sensor H. Plots shown are: a) splitting the angle between the two slops b) transition in the first derivative.

The thermomechanical testing provided insight into the changes in sensitivity, drift, noise, and sensor hysteresis. The high temperature testing schedule and the obtained results are shown for Sensor A in figure 30, where some of these effects are demonstrated. Sensor A shows excellent sensitivity throughout the temperature range with negligible noise. A notable shift is observed between the first and second tests, where the trend is not repeated between tests two and three. This shows that settling occurred in the microstructure during the initial thermomechanical load. A smaller drift can be observed in the opposite direction between tests two and three, which represents the thermomechanical drift of the sensor. In addition to the drift, the $120{ }^{\circ} \mathrm{C}$ loading sequence shows a time dependent hysteresis which can be seen throughout the loading steps and in the initial portion of the unloading steps. While this is most notable during the first test, it persists through tests two and three as well. 

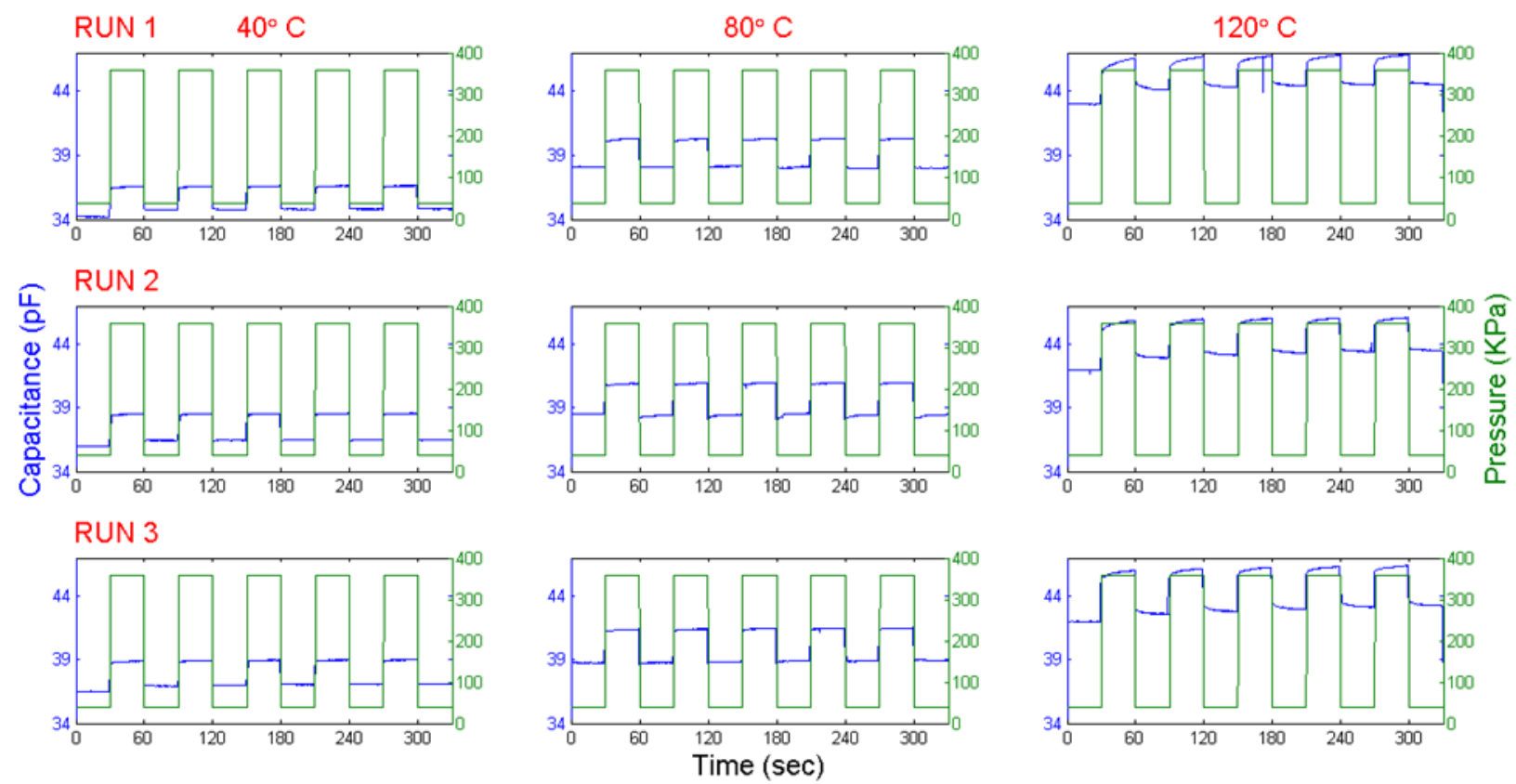

Figure 30. Thermomechanical loading schedule and response of sensor material A.

The loaded and unloaded responses were averaged for each temperature, and their difference was used to show the reponse of the sensor. The average response for tests two and three are shown in figure 31 throughout the entire temperature range. Note that the connecting lines do not represent real values, but are intended only to assist the reader with linking the points for each sensor. The reponse of Sensors A and $\mathrm{G}$ spike early, and appear to plateau at $80^{\circ} \mathrm{C}$, while the response of Sensors $\mathrm{E}$ and $\mathrm{H}$ rise continually with temperature. The remaining Sensors, B, C, D, and F, experience a small local peak in their response at $40{ }^{\circ} \mathrm{C}$ and a large spike at $120{ }^{\circ} \mathrm{C}$.

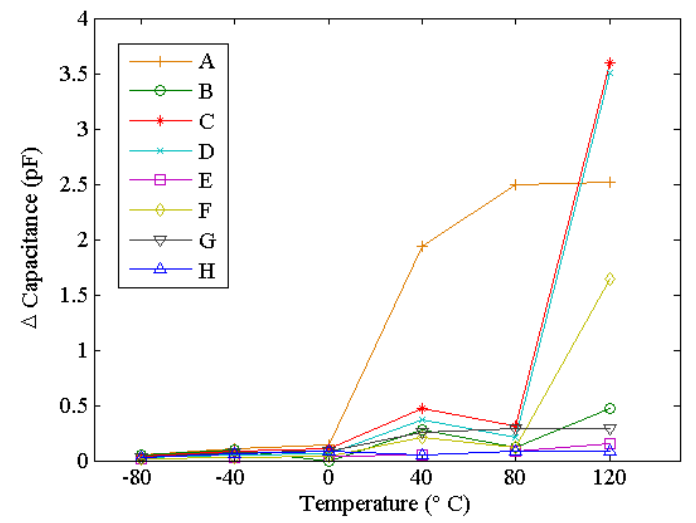

Figure 31. Sensor responses across the full temperature range. 
The intrinsic electrical properties of the composites were also tested as a function of temperature in order to compare against the findings of the thermomechanical testing. The capacitance and dielectric loss tangent values were measured at $200 \mathrm{kHz}$ (same as previous sensor testing) and are shown for the temperature range of $-40{ }^{\circ} \mathrm{C}$ to $120^{\circ} \mathrm{C}$ in figure 32. A distinct peak can be seen in the loss tangent from $40{ }^{\circ} \mathrm{C}$ to $40{ }^{\circ} \mathrm{C}$, and again as the temperature approaches $120^{\circ} \mathrm{C}$. These curves correlate with the nonlinear portion of the capacitive curve in the same temperature ranges.

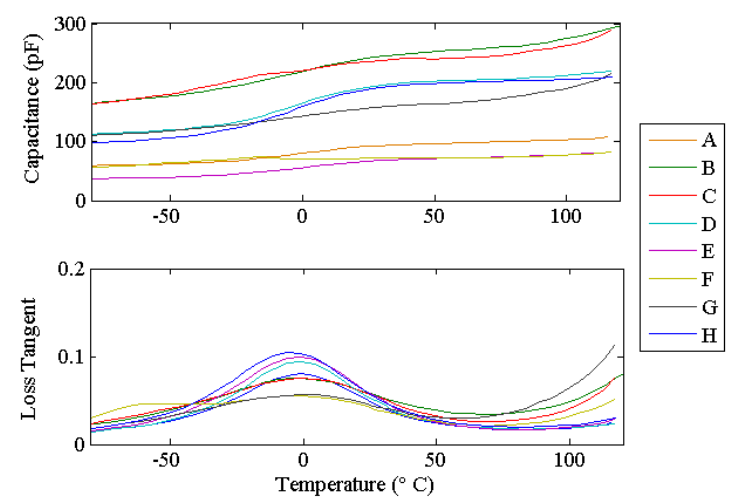

Figure 32. Capacitance and dielectric loss as a function of temperature (measured at $200 \mathrm{~Hz}$ ) for composite sensor materials A$H$.

The capacitance and dielectric loss tangent values are shown across the AC frequency spectrum at room temperature $\left(25^{\circ} \mathrm{C}\right)$ using a logarithmic axis in figure 33. There is a drop in the loss tangent at low frequencies, with a more linear trend beginning between $100-200 \mathrm{kHz}$.

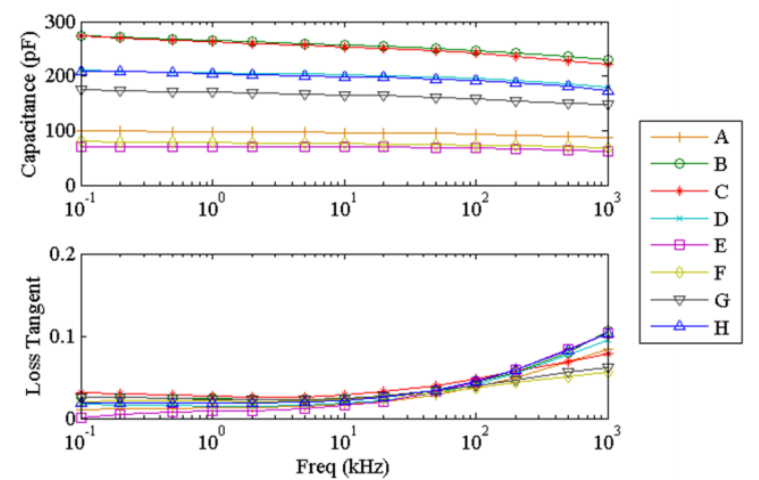

Figure 33. Capacitance and loss tangent as a function of frequency for sensor materials A-H at room temperature. 


\subsection{Discussion of Taguchi analysis}

\subsubsection{Summary of Results}

The Taguchi analysis is used to relate sensor results to individual variables. In Table 2, these results are displayed, showing the influence of each variable and each variable layer. In the Taguchi matrix there were 8 different compositions. Since each variable has two layers, there will be 4 sensors with the first layer, and 4 sensors with the second layer. Taguchi analysis works by showing the average character of the four sensors with layer 1, and the four sensor of layer two for each variable. For example, Sensors AD were $150 \mu \mathrm{m}$ thick and Sensors E-H were $300 \mu \mathrm{m}$ thick. Thus, in the sensor thickness versus capacitive baseline category, the average capacitance of Sensors A-D is shown under the $150 \mu \mathrm{m}$ subhead, and the average capacitance of Sensors E-H is shown under the $300 \mathrm{~nm}$ subhead. 


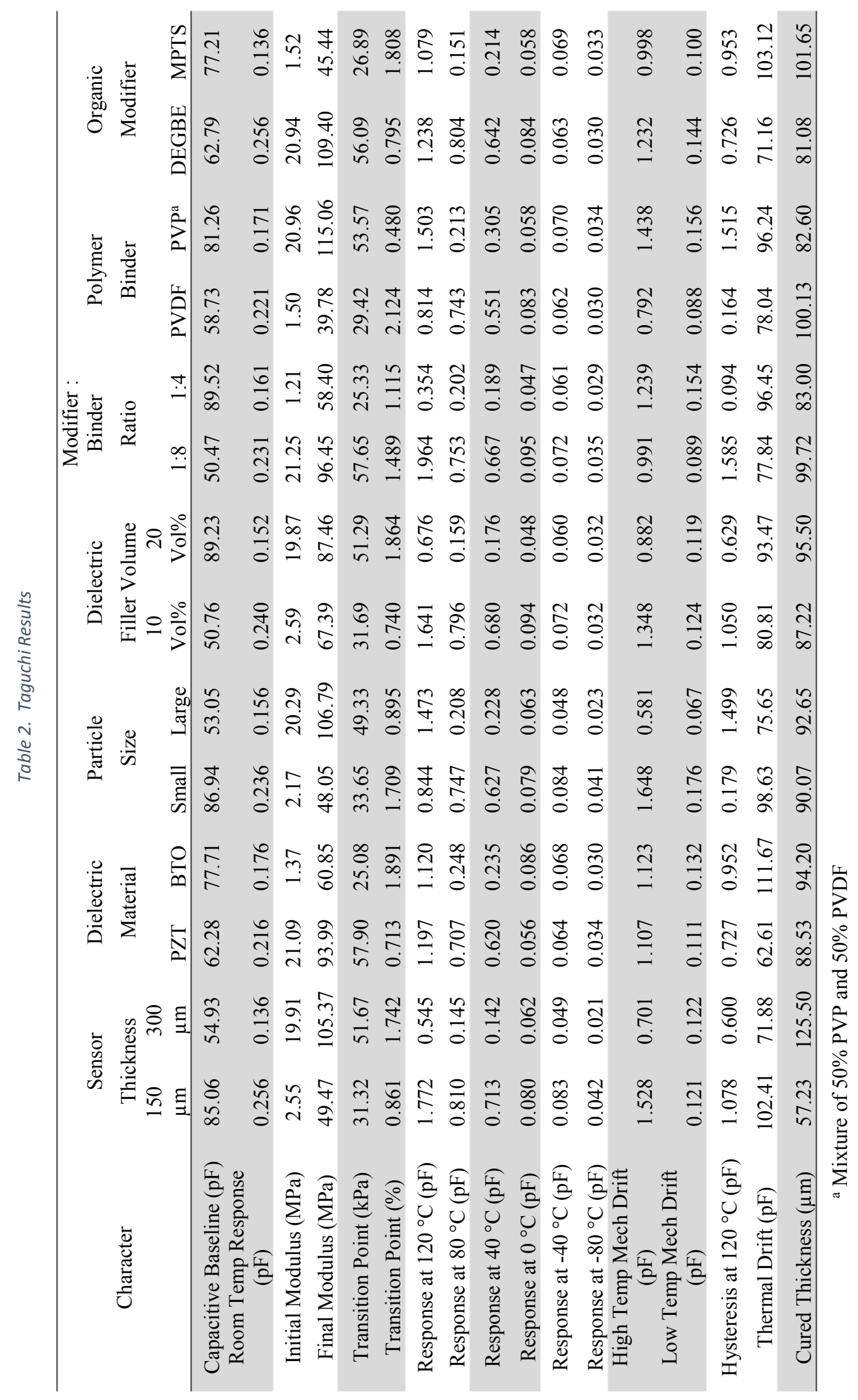




\subsubsection{Baseline capacitance}

As expected from the equation for a capacitor, a higher capacitance was found in compositions that were thinner, contained materials with a higher dielectric constant (BTO), or contained a larger volume percent of high dielectric materials. Furthermore, Dietze et al. showed that by increasing the ceramic volume content leads to an exponential rise in the capacitance [73-74]. Compositions with a smaller ceramic particle size were also found to have a higher capacitance, which is consistent with work showing a sizerelated peak dielectric constant for BTO near a particle size of $70 \mathrm{~nm}$ for room temperature testing [75]. Ferroelectric materials experience a peak in their dielectric properties at their temperature induced ferroelectric phase transitions. Materials of a specific particle size will have a set temperature at which they encounter a phase transition in their crystal structure. The phase transition where the material transitions from a ferroelectric to a paraelectric, is known as the Curie transition and is characterized by the Curie temperature. However, the Curie temperature of a material is dependent on the particle size, as smaller particles experience this phase transition at lower temperatures. This is known as the ferroelectric size effect [76].

It has been shown that polymers act as linear adsorption isotherms, where polymers with a molecular weight near that of PVP will wrap single surfactant molecules, while high molecular weight polymers, such as PVDF, are found to bind multiple surfactants molecules creating a complex matrix [77]. Here we find a similar effect in the polymer-filled composites, which are known to create aggregates in the composite microstructure [78]. This occurs as the polymers adsorb to the filler particle (or air bubbles introduced during vigorous mixing) then gradually combine during mixing to create an air- or particlefilled aggregate. The result is small and simple aggregates form with the PVP compositions, and large, complex aggregates formed with the PVDF compositions. These interactions cause the compositions containing PVP to experience a reduction in internal residual stress over the PVDF. The high molecular weight PVDF molecules also experience recoiling, introducing additional internal residual stresses [79]. These stresses are believed to be normal to the thickness, since they were heavily influenced by the drying process and adhesion of the film to the carrier substrate. Shaw et al. demonstrated that these internal residual stresses significantly reduce the capacitance of the composite film [80-81]. There are two primary sources of the converse electrostrictive effect (piezodielectric effect) which decrease the capacitance. The primary sources is the tensile stress occurring normal to the applied current, and the second is compressive stress occurring parallel to the applied current.

The molecular architecture of MPTS is a brush- or star-like form which restricts efficient organization of these molecules compared to the simpler block copolymer DEGBE. As a result, the MPTS molecules resist packing, and thus, reduce the consolidation of the composite microstructure during forming. 
Alternatively, the block-like DEGBE molecules pack more densely as they are smaller and more flexible. Consequently, films with MPTS exhibited an increased cured film thickness over those with DEGBE. While this effect (being thicker) is expected to result in a lower capacitance for films with MPTS, results were contrary to this prediction. It is believed that compositions containing MPTS may aid in the dispersion of internal residual stress, as the branched molecules provide an increased number of slip-links which are known to decrease viscosity [82-84].

Increasing the amount of modifier in the matrix increased the capacitance. This is caused by the low molecular weight materials increasing polydispersity in the polymer blend, decreasing viscosity and assisting in dispersion of internal residual stresses which resulted from numerous sources described above [85-86]. Since the internal residual stress reduces capacitance, sensors with less internal stress experience a higher baseline capacitance [80].

\subsubsection{Room temperature response}

Despite the fact that the thicker sensor materials displayed a larger displacement given a similar strain value, the magnitude of the sensor response is larger in thinner sensors. This effect is expected, since the capacitance of the thinner sensor compositions was higher. Smaller ceramic particles also resulted in a larger response, as they increased the overall capacitance of the material because of their higher dielectric constant (described in section 3.4.2).

After performing additional testing to quantify the magnitude of the sensor response to a maximum loading of $350 \mathrm{kPa}$ (without the aid of the ceramic materials), it was shown that the relationship between the sensor response and the volume of ceramic content was non-linear. The average response for 0 vol\%, $10 \mathrm{vol} \%$, and $20 \mathrm{vol} \%$ content of ceramics is shown in Table 3 . Note that the 0 vol $\%$ average would have been even lower if it had not been maximized by using the 1:8 modifier / polymer ratio and DEGBE as the modifier. 
Table 3. Composite composition versus average sensor response.

\begin{tabular}{cc} 
Ceramic Vol\% & Response $(\mathrm{pF})$ \\
\hline $0 \%$ & 0.211 \\
$10 \%$ & 0.240 \\
$20 \%$ & 0.152
\end{tabular}

In Table 3, it is found that the capacitive response was optimized between $0 \mathrm{vol} \%$ and $20 \mathrm{vol} \%$ loading of the ceramic in the polymer matrix. It is believed that the sensor response is reduced at the 0 vol\% loading of ceramic, because of a reduced dielectric constant of the bulk material. The response was reduced at $20 \mathrm{vol} \%$ loading of ceramic because of an increased compressive modulus of the bulk material. Therefore, these results follow the general trend dictated by the thought that a purely polymeric composition would result in a low capacitance, high strain sensor; a purely ceramic composition would results in a high capacitance, low strain sensor.

The sensor materials containing PVDF showed a higher response resulting from a decreased compressive modulus. This is explained in more detail in section 3.4.4. Since MPTS was found to increase the capacitance and decrease the compressive modulus of the material, it is expected that it will result in a higher capacitive response under mechanical loading. However, this is not the case for room temperature testing. It is believed that the introduction of internal stresses during mechanical loading decreases the capacitance, as did the introduction of internal residual stresses discussed in section 3.4.2 [80]. This means that mechanical loading on sensors containing MPTS will simultaneously decrease capacitance from the introduction of internal stresses while increasing capacitance from a decrease in thickness. This results in a lower overall change in capacitance as compared with sensors containing DEGBE, which has a higher internal residual stress. Increased modifier content also increased the capacitive response. This effect is caused by an increased polydispersity due to an increased molecular weight distribution of the organic materials, which is widely known to decrease the viscosity of the polymer blend and reduce the modulus composite [85-86].

\subsubsection{Compressive modulus}

As expected, the sensor materials with a greater casting thickness were found to have an increased compressive modulus. This is due to an increased level of residual stresses as a result of volume shrinkage during the drying processes. Compositions containing PZT also exhibited a higher compressive modulus. In filler:polymer compositions, changes in the compressive modulus are heavily influenced by surface preparation of the particles. It is believed that the surface chemistry of the materials 
may be the cause of this effect [87]. The most notable difference between the BTO and PZT used in this work, other than the chemical composition, is that hydrothermal synthesis of BTO gives a surface with high hydroxyl group adsorption, and the spray drying of the PZT gives a surface known to have ammonium group adsorption [88-89]. Additional surface chemistry characterization should be carried out as a part of future work.

Larger particles were found to increase the compressive modulus, which is contrary to traditional composite trends [90]. However, it has been shown that particles in the range of those used for this testing matrix have similar effects on the modulus despite their size differences [91]. Additionally, it has been demonstrated that differences in the polymer morphology surrounding the particles can have a significant effect on the modulus [87]. This may have contributed to the unpredicted results described. As is expected from use of the rule of mixtures and shown in experimental results, an increased volume of ceramic particles gives rise to the modulus [92-93].

$$
E_{c}=f E_{f}+(1-f) E_{m}
$$

Here $E$ is the elastic modulus, subscribes $c, f$, and $m$ stand for composite, filler, and matrix respectively, and $f$ is the volume fraction of the filler material.

At room temperature, the sensor compositions with a PVP polymer matrix experienced a higher modulus. This was caused by PVP being below its glass transition temperature $\left(175^{\circ} \mathrm{C}\right)$ and the PVDF being above its glass transition temperature (about $35^{\circ} \mathrm{C}$ ) [94-95]. The modulus of compositions containing PVP may have also been higher as the microstructure is predicted to be more rigid and less porous. Additionally, PVDF solutions are shown to create large air filled aggregates when air is introduced by aggressive processes of the composition [78,96]. These large, air-filled, aggregates likely form during mixing and sonic dispersion of the solution, introducing additional air pockets into an already porous structure, further decreasing the modulus.

As previously described, the simple molecular architecture of DEGBE led to an increased packing of the microstructure, resulting in a thinner, denser bulk material. The increased density led to a rise in the residual stresses, which further increases the compressive modulus. As described in section 3.4.2, increasing the modifier content will decrease viscosity of the polymer matrix. This causes the elastic modulus to lower. 


\subsubsection{Thermomechanical response}

The completed measurements showed that by changing the sensor thickness, ceramic volume loading, and polymer/modifier ratio altered the capacitive response similarly throughout the thermomechanical loading schedule over the entire temperature range $\left(-80^{\circ} \mathrm{C}\right.$ to $\left.120^{\circ} \mathrm{C}\right)$. Since the details of these effects have already been summarized in the room temperature response section, they will not be further discussed here. The effects of PZT and BTO on the sensor response are complex. The sensors response $(\Delta \mathrm{C})$ for an applied load of $350 \mathrm{kPa}$ is shown at different temperatures in Figure 34. This figure shows transitions points for both materials near $40{ }^{\circ} \mathrm{C}$ and above $120^{\circ} \mathrm{C}$.

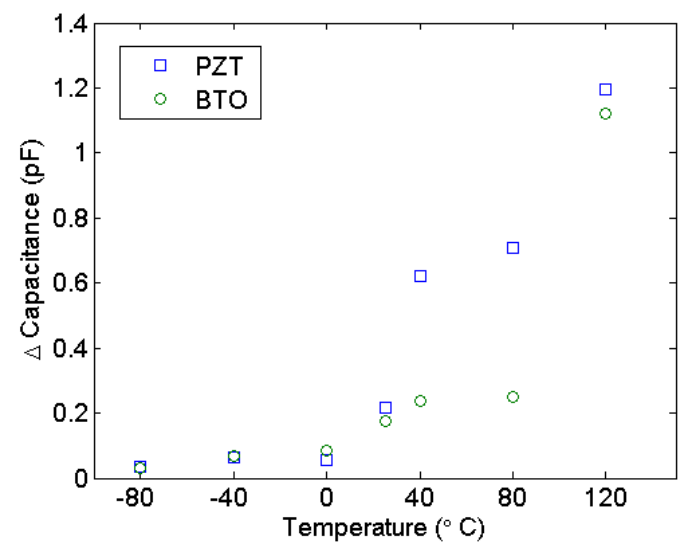

Figure 34. Capacitance response $\left({ }^{\circ} \mathrm{C}\right.$ ) as a function of temperature for sensor materials containing PZT (average of Sensors A, $B, E, F)$ and BTO (average of Sensors $C, D, G, H$ ).

This complex relationship can be best described by first outlining the Curie temperatures for the ceramic materials. As the materials approach their Curie temperature, the capacitance spikes, in turn giving higher responses from the sensors [97]. Both the small and large PZT particles have a Curie temperature of $390^{\circ} \mathrm{C}$, which is far out of the range of this application [98-99]. In contrast, BTO particles have a number of transitions temperatures in the working range. BTO particles which are 200 $\mathrm{nm}$ or larger have a Curie temperature just above $120^{\circ} \mathrm{C}$, identified by a phase transition from the paraelectric cubic phase to ferroelectric tetragonal phase when reducing the temperature to below $120{ }^{\circ} \mathrm{C}$. As the temperature is further reduced below the Curie temperature, the dielectric constant of the particles quickly drops with the exception of two spikes occurring at additional phase transitions, from the tetragonal to orthorhombic near $20^{\circ} \mathrm{C}$, and from the orthorhombic to rhombohedral phase near $-75^{\circ} \mathrm{C}$ [100-101]. Unlike the polymer matrix, the sub-micron BTO particles experience a rise in permittivity correlating with increased internal stress [102-105]. This was shown computationally by Buessem et al. 
using internal stress models and thermodynamic theory, and was further confirmed using x-ray diffraction (XRD), suggesting that the change in permittivity is accounted for as internal stresses force the crystal structure back toward the cubic phase.

While these phase transitions are communal to BTO of all sizes, the temperature of their occurrence is significantly affected by the aforementioned size effect for particles under $200 \mathrm{~nm}$. For example, particles $100 \mathrm{~nm}$ in size experience a Curie temperature (transition to the tetragonal phase) in the range of $40{ }^{\circ} \mathrm{C}[72,106]$. This introduces further transitions in the dielectric constant into the composites. These capacitive spikes account for the aforementioned trends, where BTO experienced a spike in the range of $40^{\circ} \mathrm{C}$, and another approaching just above $120^{\circ} \mathrm{C}$. The cause of the non-linear regions found with PZT are unaccounted for, though it is believed they are related to stress properties in the thin film [107].

As expected, the thermomechanical loading of the composites showed a strong dependence on the glass transition temperature of the polymer matrix materials. In the range of $0{ }^{\circ} \mathrm{C}$ to $80{ }^{\circ} \mathrm{C}$, a greater response was found with the PVDF, where the PVP had a higher response at temperatures of $-40{ }^{\circ} \mathrm{C}$ and below, and at $120^{\circ} \mathrm{C}$. The exchange between $0{ }^{\circ} \mathrm{C}$ and $40{ }^{\circ} \mathrm{C}$ is accounted for as the PVDF reaches its glass transition temperature in the range of $-35^{\circ} \mathrm{C}$, giving a quick rise to its modulus [95]. Similarly, PVP becomes more sensitive at the higher temperature of $120^{\circ} \mathrm{C}$ as it approaches its glass transition temperature of $175^{\circ} \mathrm{C}[94]$.

As explained in section 3.4.3, sensor compositions containing DEGBE had a greater response at room and higher temperatures. At temperatures of $-40{ }^{\circ} \mathrm{C}$ and lower, compositions containing MPTS showed an increased response. It is believed that the modifier material passes its glass transition temperature at this point, where the material becomes more brittle and begins to micro-crack under mechanical loading. Upon molecular separation of the MPTS molecules from the binder polymer, slip lines are introduced. This allows internal stresses to be released and reduces the rigidity of the material, leading to a lower elastic modulus. This is the source of the increased capacitance observed with MPTS at low temperatures. 


\subsubsection{Rate dependent hysteresis at $120{ }^{\circ} \mathrm{C}$}

In their amorphous state, all polymers exhibit some amount of viscoelastic character. However, since viscoelasticity is defined by time dependent behavior and is quantified by the dynamic modulus, quasistatic loading gives the appearance of a fully elastic modulus as was seen for testing at $80{ }^{\circ} \mathrm{C}$ or lower in this work. In contrast, a large rate dependent hysteresis was found during mechanical loading at $120{ }^{\circ} \mathrm{C}$ indicating that the loading scheme was no longer quasi-static. This indicates that the loading rate schedule was occurring rapid enough to affect the stress verses strain relationship. Since the velocity of the loading scheme was not altered, there must have been a transition in the composite material causing an increase in the viscous behavior of the viscoelastic polymer matrix.

Viscoelastic behavior has been shown to depend on three primary variables affecting molecular and sub-molecular cooperative motion of the polymer chains. These are the length of the polymers (represented by the average molecular weight), the mobility of the polymers, and sub-molecule/intermolecule interactions [82]. Molecules that are longer and more flexible will show an increase in sliplinks and exhibit more viscous behavior [83-84]. When thermal loading is applied to the amorphous polymer matrix, the additional energy introduced causes an increase in mobility and interactivity [108].

Thin films $(t<80 \mathrm{~nm})$ have been shown by Dalnoki-Veress et al. to exhibit a reduced glass transition temperature as the film thickness is decreased, which translates to an increased hysteresis for thinner films as higher mobility is achieved with lower energy. However, this trend is limited to thin films and does not extend to the thick films discussed in this work [84]. Rather, this work showed that hysteresis was reduced with an increase in the film thickness. Thick films $(t>10 \mu \mathrm{m})$ show an increased internal residual stress caused by a greater volume shrinkage in the drying process which reduces molecular mobility, and thus, reduces the hysteresis [109].

The BTO particles increased the magnitude of the hysteresis by simply increasing the overall capacitance of the material. The results also showed increased hysteresis, which correlates with the increase in the particle size of the filler material. This trend has been demonstrated in literature, where it was showed that well-dispersed polymer-filler systems have a significantly larger dynamic modulus phase angle for larger particles (elastic materials have a phase angle of $0^{\circ}$, and viscous materials have a phase angle of $90^{\circ}$ ) [110]. Moreover, the phase angle magnitude in viscoelastic polymer-filler composites is more significantly influenced by agglomerations in the filler material than by the particle size [111]. Since the BTO particles considered in this work to be $1 \mu \mathrm{m}$ were actually small agglomerations formed by sintering, they contributed greatly to this trend. The compositions with a higher volume content of ceramics showed decreased hysteresis at higher temperatures. This is in accordance with experimental 
results, which show that filler materials lower the magnitude of the viscoelastic spike in polymer-filler composites [112-113].

It has been shown that the phase angle of the dynamic modulus peaks at $100{ }^{\circ} \mathrm{C}$ for PVDF, and at $120{ }^{\circ} \mathrm{C}$ for PVP, indicate a transition from a glassy state to a more viscous rubbery state [94,114]. While it is known that reduced molecular weight polymers experience less hysteresis, this work showed that the sensor compositions with 50 vol\% PVP (8,000 g/mol MW)/50 vol\% PVDF (500,000 g/mol MW) experienced higher hysteresis than those containing only the high molecular weight polymer PVDF. This effect is caused by an increase in the polydispersity which decreased the viscosity of the polymer blend. This in effect increased the molecular mobility of the binder and giving rise to the overall hysteresis in the matrix [85-86]. The materials with the MPTS modifier experienced higher hysteresis as the brush-like architecture of the modifier increased intermolecular interactions and increased various slip-links [82,84].

The increased modifier content decreased hysteresis in the sensors. Using the above-mentioned criteria to evaluate the effects of the modifier content, a number of mechanisms (i.e. polymer mobility, polydispersity, density) are found to promote and depress hysteresis. The exact mechanism causing the relationship between the modifier content and hysteresis could not be definitively determined.

\subsubsection{Thermomechanical drift}

The drift caused by the thermomechanical loading was affected by similar compositional and microstructural variables at both high and low temperatures, with the exception of thickness, which had negligible effect at low temperatures. At high temperatures, the drift was reduced in thicker films, as the thicker films are known to experience increased internal residual stress which suppresses the reorganization of the microstructure. As with hysteresis, the BTO particles increased the magnitude of the drift more than the inclusion of the PZT particles by further increasing the overall capacitance of the materials.

Compositions with smaller particle sizes were found to exhibit higher drift. This is believed to be a function of the differences in the electrical properties of the materials. The composite materials consisting of conductive filler materials are shown to have non-ohmic characteristics, caused by electron tunneling between the filler particles [115]. Electron tunneling from particle to particle through the bulk material was exponentially increased by reducing the inter-particle spacing, and the inter-particle spacing was linearly decreased by reducing the particle size. This was similarly found in other works [116]. Thus, reducing the particle size exponentially increased the effects of electron tunneling in the polymerfiller composites, resulting in a reduction in the effective percolation threshold. 
The compositions in this work do not include conductive fillers; however, ferroelectric particles produce a similar effect with the electrical flux of the composite. Rather than affecting conductivity, reduced inter-particle spacing was found to affect charging of the bulk material by increasing electric flux [117-118]. This causes compositions with smaller particles to experience greater changes in charging as the effects of electrical flux are more significant. Additionally, smaller particles are more likely to be displaced in the composite microstructure causing compositions to experience increased changes in the electric flux, making them more susceptible to cause drift. Drift was also more pronounced when less ceramic materials were used, as the composite is more heterogeneous and the particle shifting causes more severe changes in the electrical flux.

The molecular weight of the polymers directly affected the shifting and reorganization of the microstructure. It has been shown that polymers with a molecular weight near that of PVP $(8,000 \mathrm{~g} / \mathrm{mol}$ MW) will wrap single surfactants, while high molecular weight polymers such as PVDF (500,000 g/mol MW) are found to bind multiple surfactants creating a complex matrix [77]. Here we find a similar effect in the ceramic-filled polymer composites, which are known to contain agglomerated particles within the composite microstructure [78]. The result is small, simple aggregates forming within PVP matrix, and large, complex aggregates forming in the PVDF matrix. In this work, composites composed of the PVDF matrix showed aggregates in the range of $2 \mu \mathrm{m}$, as seen in figure 27. These more complex aggregates reduce reorganization of the microstructure, thus reducing drift. Another important contribution to drift is the addition or removal of residual stresses during reorganization of the softened microstructure during the thermomechanical process. The modifier MPTS is known to promote adhesion between polymers, and therefore, reduce drift over DEGBE modifier. The drift was further reduced by decreasing the content of the modifiers, which increased the mobility of the binder molecules.

\subsubsection{Characterization of thermal affects on capacitance}

The non-linear dielectric character under thermal loading identified in this work was consistent with similar films tested by Furukawa et al. Smaller casting thicknesses increased the thermal drift, which is greatly influenced by changes in the crystalline structure [119]. It is believed that thinner composite films experience crystalline changes more quickly and more severely, influencing the response from the dynamic temperature sweep. Compositions containing BTO experienced a higher change in capacitance under thermal load (thermal drift), especially those with a smaller particle size. This correlates with the Curie temperature being in the center of the temperature scheme for $100 \mathrm{~nm}$ BTO particles, just outside of the testing range for particles over $200 \mathrm{~nm}$, and far outside the range for both PZT particle sizes [72,98- 
99,106]. Also, compositions with a higher ceramic content displayed a higher thermal drift, as the ceramic particles are the main source of the drift.

The same polymer and modifier variables that were found to increase the baseline capacitance were also found to increase thermal drift. It is believed that the introduction of internal stresses resulting from thermal loading more significantly affected the variables that promote low residual stress. This is because the low stress compositions showed a greater change in their internal stress upon the introduction of thermal stresses.

\subsection{Sensor Design}

Using the conclusions from this work, a robust ceramic/polymer composite sensor material can be engineered. This design considers not only the linear trends of the Taguchi analysis, but also takes into account the more complex trends found in literature to affect the results of the testing matrix. The following statements summarize the key findings of this work:

- The thickness of the sensor should be minimized to increase the sensor response and simplify potential future incorporation into a secondary structure or mechanism. Reduction of the film thickness is limited by a loss in strength, as the sensors should be easily handled without being damaged. Disadvantages include increases in the drift magnitude.

- High dielectric ceramic materials should be evaluated by their dielectric constant, Curie temperature, and wetting characteristics. Moving the Curie temperature towards $-80^{\circ} \mathrm{C}$ will increase the low temperature response of the sensors, while drawbacks of this approach include an increase in the temperature drift of the sensor. Increasing the Curie temperature well above the working range would reduce this temperature drift.

- The particle size of the ceramic materials will be partially determined by placement of the Curie temperature. Smaller particles will increase the capacitance by increasing the electric flux percolation. Additional considerations include decreasing the compressive modulus by decreasing the particle size, without significantly increasing drift caused by reorganization of nano-particles in the microstructure.

- The ideal volume content of ceramic materials is most heavily influenced by its relation to the sensor response through its effects on the dielectric constant and modulus of the bulk material. These variables are optimized near a 10 vol\% loading of the ceramic materials (for this particle size range). 
- A polymer/modifier matrix should be selected with a melting temperature exceeding the thermal range of the application. Maintaining the elastic properties of the matrix throughout the required thermal range will eliminate hysteresis. The matrix should also have a low modulus, a high molecular weight, and a complex molecular architecture to reduce drift and should meet ASTM E595 outgassing standards.

Design of the final sensor package and embedding technique into other structures should be considered during processing of the sensor composition. The sensor package, including electrodes and insulating coatings, should be thin, flexible, and similarly resistant to thermal effects. While variations in sensor packaging are infinite, one example is shown in figure 35. This sensor package was fabricated on a Kapton film substrate, having a $10 \mu \mathrm{m}$ thick composite sensor composed materials including Huntsman controlled volatility polymers and PZT. Platinum electrodes were used to prevent oxidation of the nanofoil. The complete sensor package has thickness of $50 \mu \mathrm{m}$.

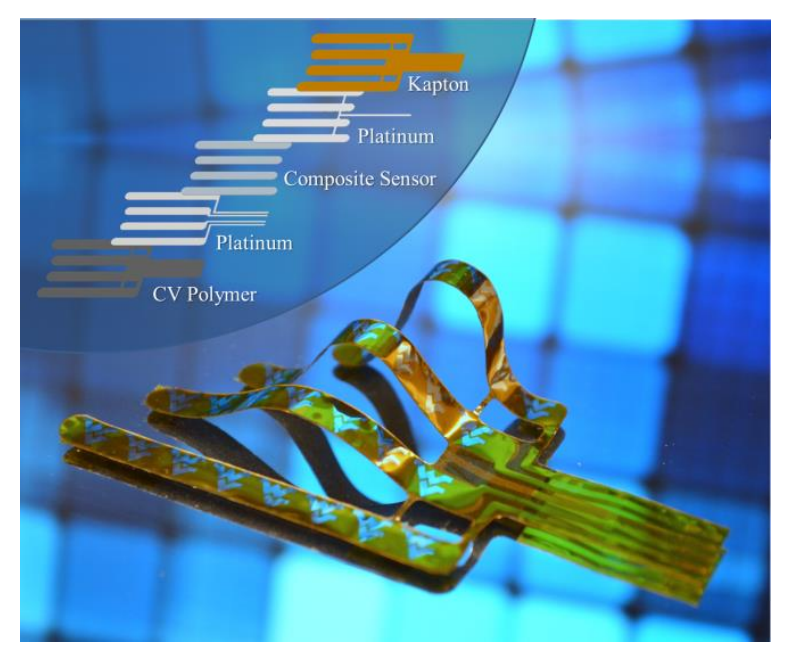

Figure 35. Sensor prototype fabricated to demonstrate use of the proposed smart film technology.

\subsection{Conclusions}

In this work, a two level Taguchi experimental design was used to prepare a test matrix, evaluating the effects of seven different compositional variables on a ceramic/polymer microstructure and sensor response. While the two layer design is limited by linear trends in the results, the mechanisms affecting the results were evaluated from literature and the non-linear trends were presented and discussed. A material selection criterion was described for the proper sensor composition for future sensor package 
designs concerning embedded force sensor arrays for harsh environment applications. The work showed that the sensor response was maximized by reducing film thickness, increasing the dielectric constant, and decreasing the compressive modulus. These goals are optimized with high dielectric ceramics using a volume loading in the range of $10 \%$, and matrix materials with molecular architectures which promote the introduction of compressive strain.

The negative effects of drift can be minimized by increasing the sensor thickness, increasing the ceramic particle size, using matrix materials with a high molecular weight and a complex molecular architecture, and by the introduction of residual stress in the films. At high temperatures, a rate dependent hysteresis was found to occur due to increased molecular mobility of the polymer matrix. This can be prevented by selecting polymers with transitions temperatures further outside of the operating temperature range. 


\section{Chapter 4. An Analytical Model for Porous Polymer-Ceramic Capacitive Pressure Sensors}

\subsection{Introduction}

An effort to obtain a better understanding of design variables has often led to analytical modeling, and finite element analysis (FEA) using computer aided designs (CAD) in recent literature. Various capacitive pressure sensors based on an open hollow membrane, or closed air-filled cavity, have been designed using these theoretical approaches with great success [120-123]. More complex PVDF - PZT (lead zirconium titanate) composites have also been modeled to predict the effect of PZT content on the permittivity, dielectric loss, piezoelectric coefficient, and elastic modulus [124-126]. Theoretical predictions of the piezoelectric effect were also made, showing the two-phase composites response to temperature [127-128]. However, models for predicting thermo-mechanical loads on porous polymerfilled composites are lacking.

This work combines a number of analytical approximations to create a comprehensive model of the three-phase pressure sensor under mechanical and thermal loading. An example microstructure is depicted in figure 36, using a scanning electron microscope micrograph. The model assumes a two-phase polymer-ceramic solid, which contains a third phase of inter-connected porosity. In this way, the elastic modulus is presented in two parts. First, the polymer-filled solid material is presented and then the solidporous material. The effects of stress from pressure, temperature, and substrate pinning on the permittivity of the constituents are considered, and the permittivity of the bulk composite is presented as a three-phase system. Finally, the change capacitance of the bulk material is presented as an iterative function of the applied pressure and temperature. 


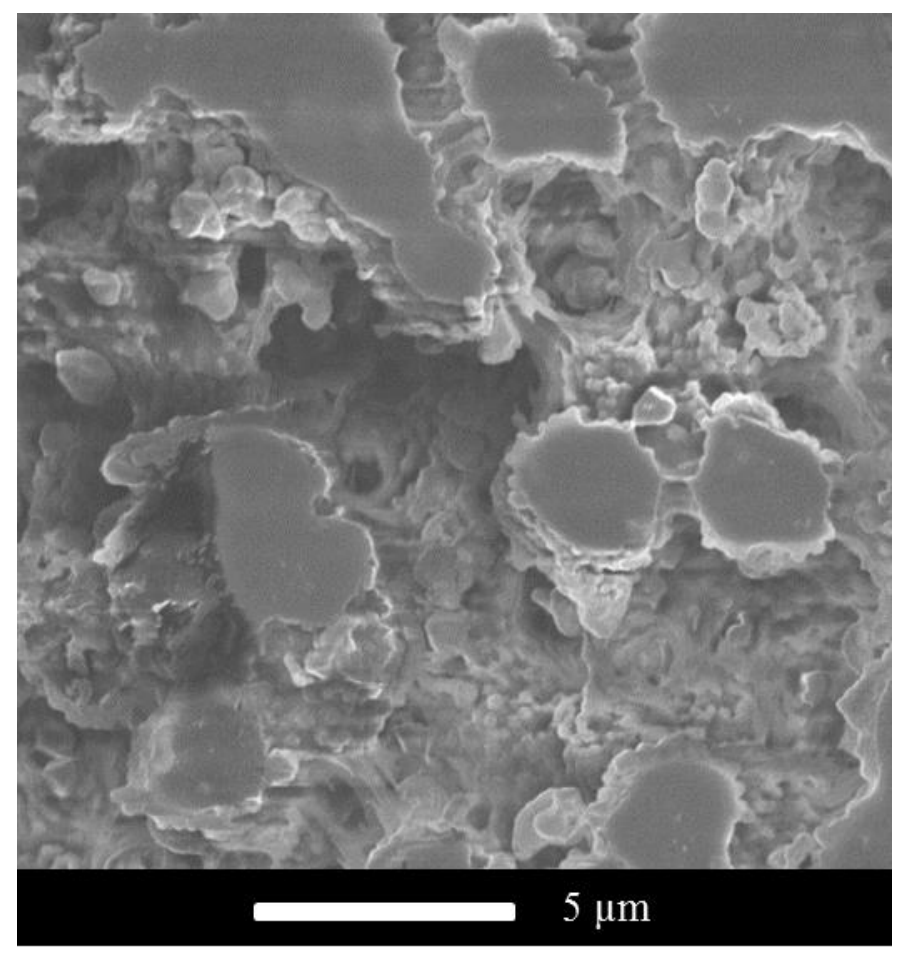

Figure 36. SEM micrograph of sensor A (composition details listed in Table 4).

\subsection{Analytical Model}

\subsubsection{Capacitance}

A capacitor operates by holding charge within a dielectric region between two electrodes. The equation for a capacitor is

$$
C=\frac{\varepsilon_{r} \varepsilon_{0} A}{d}=\frac{\varepsilon A}{d}
$$

where $C$ is the capacitance, $\varepsilon_{r}$ is the relative permittivity (dielectric constant), $\varepsilon_{0}$ is the permittivity of free space, $A$ is the surface area, and $d$ is the thickness of the capacitor. The working mechanism in a capacitive sensor is a change in thickness introduced by strain, calculated by

$$
C_{1}-C_{2}=\frac{\varepsilon_{r} \varepsilon_{0} A}{d_{1}}-\frac{\varepsilon_{r} \varepsilon_{0} A}{d_{2}} .
$$




$$
\Delta C=\frac{\varepsilon_{r} \varepsilon_{0} A \epsilon}{d_{2}} .
$$

Here, $\epsilon$ is strain, $d_{1}$ is the pre-strain thickness, and $d_{2}$ is the post-strain thickness. Since $d_{2}$ is not known $a$ priori, it should be calculated by

$$
d_{2}=\left(\frac{d_{2}-d_{1}}{d_{1}}\right) d_{1}+d_{1}=\epsilon d_{1}+d_{1}=d_{1}(1+\epsilon)
$$

so that the capacitive response becomes

$$
\Delta C=\frac{\varepsilon_{r} \varepsilon_{0} A \epsilon}{d_{1}(1+\epsilon)}=\frac{\varepsilon_{r} \varepsilon_{0} A}{d_{1}(1+1 / \epsilon)}
$$

Since the sensor is being used to measure pressure, not strain, the elastic modulus will be used to translate between the two variables providing

$$
\Delta C=\frac{\varepsilon_{r} \varepsilon_{0} A}{d_{1}(1+E / \sigma)}
$$

The variable $E$ is the elastic modulus, and $\sigma$ is the applied stress. In this equation, both the relative permittivity and elastic modulus are not known intrinsically, since the capacitor is a composite.

\subsubsection{Elastic Modulus}

For this work, it is assumed that the polymer matrix is selected such that its melting temperature is sufficiently higher than the working temperature range, preventing the effects of viscoelasticity. Thermal and mechanical loading is assumed to be sufficiently low to prevent plastic deformation, and changes in the surface area, $A$, are assumed negligible.

Early work on the elastic modulus of a two-phase system was performed by Hill in 1963 [129]. One finding of great significance was a modification to his previous work, where he modeled the elastic modulus of a fiber-reinforced matrix assuming transversely isotropic behavior about the fiber, which is perfectly aligned along a single axis. This assumption is further detailed by stating an assumed statistical homogeneity for the spatial arrangement of fibers in both the longitudinal and transverse directions [130- 
131]. Using assumptions by Kerner concerning the physical relations in composites, Herman modified Hill's elastic equations to a more simple form in 1966 [132-133].

Further yet, in 1969 Halpin and Tsai created an analytical version of Herman's equations which allows for geometries other than the aligned continuous fibers [131,134]. This work uses the M. van Es modified Halpin-Tsai model developed in 2000, which modifies the aspect ratio giving a more accurate analytical model for predicting the elastic modulus of the polymer-filler composite by [135]

$$
\begin{gathered}
E_{c}=\frac{E_{m}\left(1+\zeta \eta V_{f}\right)}{1-\eta V_{f}} \\
\text { where: } \eta=\left(\frac{E_{f}}{E_{m}}-1\right) /\left(\frac{E_{f}}{E_{m}}-\zeta\right) \\
\zeta=\frac{2 w}{3 t} .
\end{gathered}
$$

In these equations, $E_{c}$ is the modulus of the composite, $E_{m}$ is the modulus of the matrix, $E_{f}$ is the modulus of the filler, and $w / t$ is the aspect ratio of the filler. The variable $V_{f}$ is the volume fraction of the filler in the solid solution (not the volume fraction of the three-phase system).

The elastic modulus is further modified with the introduction of pores into the composite material. This is done by considering the polymer-ceramic composite to be a single phase, having the properties previous approximated. Traditionally the modulus of a porous material is characterized by the Spriggs equation developed in 1961

$$
E=E_{0}^{-b V_{p}}
$$

where $E_{0}$ is the non-porous modulus, $b$ is a material constant, and $V_{p}$ is the volume percent of porosity [136]. However, this empirical model fails to predict the elastic modulus for materials with a high porosity and was thus updated by Wang in 1984 [137]. Wang's model (shown below) used a polynomial exponent achieving accurate approximations for highly porous materials, though the length of the required polynomial diminished the practical significance of the model, shown by

$$
E=E_{0} \exp \left[-\left(b V_{p}+c V_{p}^{2}+d V_{p}^{3}+\cdots\right)\right]
$$


Within Wang's expression, $b, c, d$, etc... are material constants. In 1987, Phani and Niyogi created a more practical model which satisfies the condition that the elastic modulus is identical to the bulk material without the presence of porosity, and the elastic modulus is zero with a fully porous material [138]

$$
E=E_{0}\left(1-a V_{p}\right)^{n}
$$

In this equation, $a$ is a material constant known as the geometry packing factor. This constant relates to the spatial organization of the material, and $a=1 / \mathrm{P}_{\text {crit }}$, where $\mathrm{P}_{\text {crit }}$ is the critical porosity where the elastic modulus drops to zero. For polycrystalline materials having a spherical geometry, the constant ' $a$ ' falls in the range of $1 \leq a \leq 3.85$. For reference, packing arrangements of cubic, orthorhombic, and rhombohedral uniformity result in values of 2.10, 2.52, and 3.85, respectively [138] [139]. The material constant $n$ relates to the interconnectivity and morphology of the pores. Materials which are systematically arranged, such as closed pores with spherical symmetry, have been modeled well with $n=2.14$. Interconnected pores that are randomly dispersed through a microstructure and have an irregular morphology are better modeled with $n=4.12$ [138].

A similar equation was proposed by Gibson and Ashbury in 1988, which was used to estimate the elastic modulus of foams by their density [140]

$$
E=E_{0} C\left(\frac{\rho}{\rho_{0}}\right)^{n}=E_{0} C\left(1-V_{p}\right)^{n}
$$

The $\rho$ is the apparent density, and $\rho_{0}$ is the density of the non-porous material. As with equation (12), $C$ and $n$ are material constants relating to the microstructure. Applied to foams $\left(90 \%<V_{p}<95 \%\right)$ with dense boundaries, it has been found that $C \approx 1$ [140-141]. Similar to ceramic materials, foams have been modeled by $1 \leq n \leq 4$, with $1 \leq n \leq 2$ used for low $V_{p}$, with closed porosity throughout the material [142].

In 2002, Zhang et al. proposed a similar three-phase elastic modulus model [143]

$$
E_{p c}=E_{c}\left(1-\left(V_{p}+V_{p f}\right)\right)^{n}
$$

where $E_{p c}$ is the porous composite modulus, $V_{p f}$ is the volume fraction of filler in the pores (in addition to the filler in the matrix). In 1960, Paul gave a two-phase composite modulus approximation 


$$
\begin{gathered}
E_{c}=E_{m}\left[\frac{1+(m-1) V_{f}^{2 / 3}}{1+(m-1)\left(V_{f}^{2 / 3}-V_{f}\right)}\right] \\
\text { where }: m=\frac{E_{f}}{E_{m}}
\end{gathered}
$$

which was simplified for filled polymers

$$
E_{f}=E_{m}\left(1+\frac{V_{f}}{1-V_{f}^{1 / 3}}\right)
$$

and porous polymers

$$
E_{p}=E_{m}\left(1-V_{p}^{2 / 3}\right)
$$

by Ishai and Cohen in 1967, with $E_{p}$ being the modulus of the porous polymer [144]. In 2004, Zhang et al. proposed a three-phase model based on these approximations [145]

$$
E_{p c}=E_{m}\left[1+\frac{\frac{V_{f}}{1-\left(V_{p}+V_{p f}\right)}}{\frac{m}{m-1}-\left(\frac{V_{f}}{1-\left(V_{p}+V_{p f}\right)}\right)^{1 / 3}}\right]
$$

While a number of equations have been proposed to model various types of porous materials, the result of each equation yields a single value equating either the original or mean elastic modulus to the original porosity [138,145-146]. However, this work requires a model which can predict the changing elastic modulus throughout the loading cycle. Experimental data from Weadon et al.is plotted in figure 37, showing the trend of four different sensor compositions (compositions shown in Table 4). The x-axis displays the estimated change in porosity under compressive stress up to $350 \mathrm{kPa}$, assuming all strain results in the collapse of the porosity within the material. The y-axis displays the experimental elastic modulus divided by the estimated non-porous elastic modulus. The non-porous modulus was approximated using (7). The result is a normalized elastic modulus, where 0 vol\% porosity results in $E_{p d} / E_{c}=1$. Note that the character of sensors $\mathrm{A}$ and $\mathrm{H}$, and that of sensors $\mathrm{D}$ and $\mathrm{E}$ were found to be very 
similar with the exception of $V_{0}$ and $V_{\Delta}$. Each pair of related sensors shares a similar organic modifier content, which is also the primary difference between the two sets.

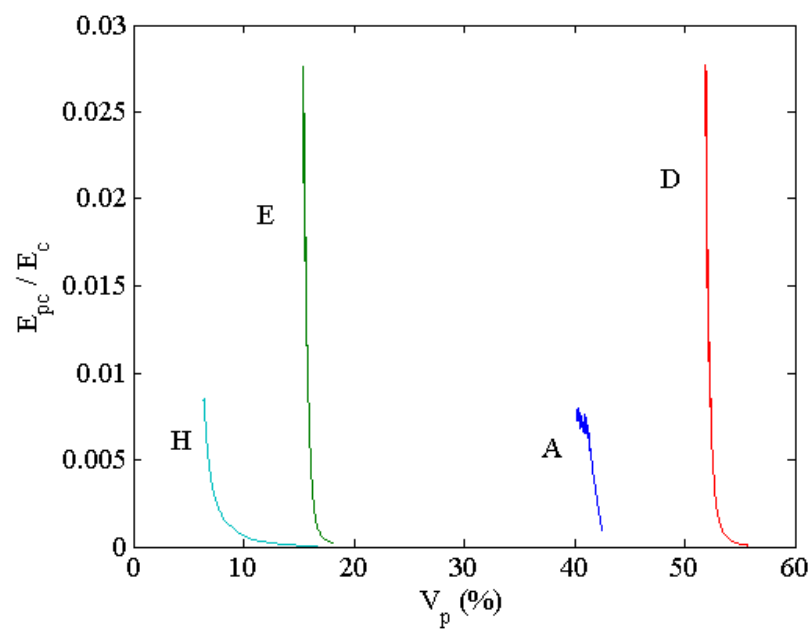

Figure 37. The normalized elastic modulus of the porous material plotted against the change in porosity under an applied load $0 \rightarrow 350 \mathrm{kPa}$.

Combining (12) and (13) as a starting point gives material constants $C, a$, and $n$, resulting in the equation

$$
E_{p c}=E_{c} C\left[1-a\left(V_{p}\right)\right]^{n}
$$

Table 4. Composition of Experimental Sensors

\begin{tabular}{cccccccc}
\hline \hline Sensor & $V_{0}(\%)$ & $d_{l}(\mu \mathrm{m})$ & Filler & $V_{f}(\%)$ & Binder & Modifier & Modifier Vol. (\%) \\
\hline $\mathrm{A}$ & 42.5 & 55.8 & $\mathrm{PZT}$ & 10 & PVDF & DEGBE $^{\mathrm{a}}$ & 11.25 \\
$\mathrm{D}$ & 55.6 & 55.6 & $\mathrm{BaTiO}_{3}$ & 20 & PVDF & DEGBE $^{\mathrm{a}}$ & 20 \\
$\mathrm{E}$ & 18.1 & 130.5 & $\mathrm{PZT}$ & 10 & $\mathrm{PVDF}$ & MPTS $^{\mathrm{b}}$ & 22.5 \\
$\mathrm{H}$ & 16.6 & 158.6 & $\mathrm{BaTiO}_{3}$ & 20 & $\mathrm{PVDF}$ & MPTS $^{\mathrm{b}}$ & 10 \\
\hline \hline
\end{tabular}

Weadon et al.

The data in figure 37 is not completely accurate, as some of the observed strain was found to be flattening of the wavy films, and thus does not correlate with collapsing pores [147]. The trends are important, revealing that the apparent critical porosity $\left(P_{c r i t}\right)$ is a function of the initial porosity. The 
character of the curves is approximated with the relation $1 / a=P_{c r i t}=V_{0}$, where $V_{0}$ is the original porosity. Simple substitution gives

$$
E_{p c}=E_{c} C\left[1-\left(\frac{1}{V_{0}}\right)\left(V_{p}\right)\right]^{n}
$$

In figure 37, the modulus of the composite also appears to approach $\mathrm{E}_{\mathrm{c}}$ before $V_{p}=0$. This is accounted for by shifting the actual porosity to an apparent porosity using the constant $P$, where $P$ is the final porosity at which the bulk material appears to approach $E_{c}$, giving

$$
E_{p c}=E_{c} C\left[1-\left(\frac{1}{V_{0}-P}\right)\left(V_{p}-P\right)\right]^{n}
$$

This can be simplified by considering the change in volume, $V_{\Delta}$, such that $\mathrm{P}=V_{0}-V_{\Delta}$. Previous literature has shown that $C \approx 1$, yielding

$$
E_{p c}=E_{c}\left(\frac{V_{0}-V_{p}}{V_{\Delta}}\right)^{n}
$$

The rate of the increasing elastic modulus as a function of strain has a strong correlation with the empirical value $\mathrm{V}_{\Delta}$. However, a direct mathematical relationship could not be established because of the previously described inaccuracy in the stress versus strain curve from flattening of warped films (especially film $\mathrm{H}$ ). The surface of the films has been prepared with a metallic electrode (described in section 3.2.2.), the electrical acquisition avoids consideration of the effect of the warped film. Since capacitance a direction function of strain, the capacitive versus stress data was used to estimate $\mathrm{V}_{\Delta}$ more accurately. Further work should be performed in this area to give a more direct mathematical correlation between the rate of the increasing elastic modulus as a function of strain, and the empirical value $V_{\Delta}$. Pending a more complete evaluation of this variable, the results of this work are only valuable as qualitative trends and cannot be confidently used as quantitative trends.

In the iterative form, $E_{p c}$ and $V_{p}$ are interdependent and $V_{p}$ is a function of the stress, such that

$$
E_{p c}^{i}=E_{c}\left[D+\left(\frac{V_{0}-V_{p}^{i}}{V_{\Delta}}\right)^{n}\right]
$$




$$
\begin{gathered}
\text { where: } V_{p}^{i}=V_{p}^{i-1}-\Delta V_{i} \\
\Delta V_{i}=A d_{i}-A d_{i+1}=-A d_{i} \epsilon_{i}=-A d_{i}\left(\frac{\sigma}{E_{p c}^{i}}\right) \\
d_{i}=d_{i-1}-\left(\frac{\sigma}{E_{p c}^{i-1}}\right) .
\end{gathered}
$$

In (25) and (26), the initial thickness is $d_{l}$, and $\sigma$ is the iterative load application. Because of the interreliant nature of the terms, finite stress iterations are required. Since $V_{p}{ }^{i}=\mathrm{V}_{0}$ when $i=1$, a small constant, $D$ must be added to prevent $\mathrm{E}_{\mathrm{pc}}{ }^{\mathrm{i}}=0$. The size of this constant depends on the iteration size of the applied stress. For this work, $0 \mathrm{~Pa} \geq \sigma \geq 350,000 \mathrm{~Pa}$, with $\Delta \sigma=10^{-3} \mathrm{~Pa}$, and $\mathrm{D}=10^{-9}$. For this iteration size, larger values of $D$ resulted in negligible differences, and $\mathrm{D}=10^{-11}$ resulted $\mathrm{E}_{\mathrm{pc}}^{\mathrm{i}} \approx 0$ for all $i$. The variables $V_{p}^{i}, \Delta V_{i}$, and $d_{i}$ are defined in the equations.

\subsubsection{Thermal and Stress Effects on Permittivity}

The permittivity of a bulk, stress-free material at room temperature will experience notable changes when pinned to a substrate and subjected to thermo-mechanical loading. The composite material in this work will be modeled by considering changes in permittivity of the polymer matrix and ceramic filler independently.

The polymer matrix is assumed to lack any ferroelectric properties. For this reason, changes in polarization caused by thermally induced phase transformations of crystalline domains within the structure are not considered. The amorphous polymer matrix is assumed to be an isotropic, statistically homogenous material. This assumption results in a bulk polymer in a paraelectric state which lacks piezoelectric properties even below the Curie temperature [148]. In reality, processing variables can introduce traces of polarization into the ferroelectric polymer; however, these are small and considered negligible [149].

The polymers will experience polarization caused by external stresses through the converse electrostrictive effect, or the piezodielectric effect. Phenomenological theory of the piezodielectric effect was first established by Osterberg and Cookson in 1937 [150]. In a single crystal, it causes tensile stress occurring normal to the plane of accumulated charge. The application of a compressive stress parallel to the plane of accumulated charge results in decreased permittivity between the electrodes due to the alteration in polarization normal to the electrodes. The effects of tensile stress acting in both axis normal 
to the charge in a single crystal were characterized by Devonshire, and demonstrated by Shaw et al. with the expression [151]

$$
\frac{1}{\varepsilon_{f}}=\frac{1}{\varepsilon_{u}}-4 Q_{12} \sigma
$$

where $\varepsilon_{f}$ is the permittivity under stress, $\varepsilon_{u}$ is the permittivity without stress, and $Q_{12}$ is the electrostrictive constant for stresses occurring normal to the charge plane. The amorphous polymer matrix has a single, unidirectional electrostriction constant, $\kappa$. Substituting the electrostriction constant, considering a single axis, and solving for $\varepsilon_{f}$ gives

$$
\varepsilon_{f}=\frac{\varepsilon_{u}}{1-2 \varepsilon_{u} \kappa \sigma}
$$

Strain induced by thermal expansion requires consideration which includes the contributions from the polymer matrix, the ceramic filler, and porosity. It is assumed that the pores are at least partially interconnected, and thus are treated as voids rather than compressed air under thermal and mechanical loading. For a sufficiently thin film, the bulk material is assumed to take on the constraints of the film surface. In effect, the film is assumed to be fully bound by the substrate on which it is adhered. Despite the presence of pores (voids) in the composite, the composite is bound by the substrate deeming the contribution of the pores negligible on the longitudinal stress and strain of the material. Thermally induced stress

$$
\sigma_{\text {Thermal }}=2 \sigma_{\text {longitudal }}=2 E_{c}\left(\epsilon_{s}-\epsilon_{c}\right)
$$

can thus be reduced to the difference between strain in the composite

$$
\epsilon_{c}=\left[\alpha_{f} V_{f}+\alpha_{m}\left(1-V_{f}\right)\right] \Delta T
$$

and strain in the substrate

$$
\epsilon_{s}=\alpha_{s} \Delta T
$$


occurring along both longitudinal axes. In these equations, $\epsilon_{\mathrm{c}}$ and $\epsilon_{\mathrm{s}}$ are the strain in the composite and substrate, and the coefficient of thermal expansion for the filler, matrix, and substrate are $\alpha_{f}, \alpha_{m}$, and $\alpha_{s}$ respectively.

Assuming that loads applied to the sensor face are applied with constant force/pressure rather than constant displacement/strain, thermal expansion in the transverse direction will not introduce any additional internal stress. This assumption is valid for applications where forces are applied with gravity, pneumatic pressure, hydraulic pressure, electrical current driven motors, or human interaction. The modified permittivity of the polymer matrix thermal-mechanical stress $\left(\varepsilon_{l f}\right)$ is given by

$$
\varepsilon_{1 f}=\frac{\varepsilon_{1 u}}{1-2 \varepsilon_{1 u} \kappa\left(\sigma_{\text {Thermal }}+\sigma_{\text {Mechanical }}\right)} .
$$

Ceramic ferroelectric thin films are known to experience a diffuse phase transition caused by pinning of the ferroelectric domains by the substrate and fine granular structure [152-153]. However, the ferroelectric ceramic in this work was bound within a polymer matrix, which has a very low elastic modulus. It is assumed that external thermal and mechanical stresses applied to the dense ceramic filler resulted in negligible changes to the permittivity [154]. Changes in permittivity do result from thermally induced phase changes in the ceramic material, which in the parraelectric phase are commonly approximated using the Curie-Weiss law. In the ferroelectric phase, spontaneous polarization is approximated using Gibbs free energy equation

$$
\begin{gathered}
\frac{1}{\varepsilon_{2 t}}=\alpha_{1}+3 \alpha_{2} P_{S}^{2}+5 \alpha_{3} P_{S}^{4} \\
P_{S}^{2}=\frac{-\alpha_{2}+\left(\alpha_{2}^{2}-4 \alpha_{1} \alpha_{3}\right)^{1 / 2}}{2 \alpha_{3}}
\end{gathered}
$$

with $\mathrm{P}_{1}^{2}=\mathrm{P}_{2}^{2}=0$, and $\mathrm{P}_{3}^{2}=\mathrm{P}_{\mathrm{s}}^{2} \neq 0$ [155-156]. Here the $\alpha$ terms are the dielectric stiffness coefficients, $\varepsilon_{2}$ is the permittivity of the ceramic filler, and $P_{s}$ is the spontaneous polarization. This equation is made a function of temperature by considering the effect of temperature on the dielectric stiffness coefficients. Typically, only the first-order term, $\alpha_{l}$, is considered to be temperature dependent [156]. Use of the CurieWeiess law gives

$$
\alpha_{1}=\frac{T-T_{c}}{2 \varepsilon_{0} C}
$$


where $T$ is the observed temperature, $T_{c}$ is the Curie temperature, and $C$ is the Curie constant. By substituting equation (35) into equations (33) and (34), the permittivity in the ferroelectric phase can be determined as a function of temperature.

\subsubsection{Permittivity of Composite}

The permittivity of ferroelectric composites is often estimated using mixing rules. These approximations take into account the permittivity and volume loading of the two-phase system, with some models including additional predictions, such as average electric fields known to influence the permittivity of composites with a high ceramic filler content [157-158]. Much work has been performed in this area, with early contributions given by Clausius, Mossotti, Lorenz, and Lorentz [158-162]. Rayleigh's mixing law predicts the influence of a cubic array of spherical objects within a secondary medium [163]

$$
\frac{\varepsilon_{c}-\varepsilon_{1}}{\varepsilon_{c}+2 \varepsilon_{1}}=V_{f} \frac{\varepsilon_{2}-\varepsilon_{1}}{\varepsilon_{2}+2 \varepsilon_{1}}
$$

where $\varepsilon_{c}, \varepsilon_{1}, \varepsilon_{2}$, are the permittivity of the composite, polymer matrix, and ceramic filler respectively, and $V_{f}$ is the volume fraction of the filler. Written in explicit form for the permittivity of the composite, it is known as the Maxwell Garnett equation [158,164]

$$
\varepsilon_{c}=\varepsilon_{1}+3 V_{f} \varepsilon_{1} \frac{\varepsilon_{2}-\varepsilon_{1}}{\varepsilon_{2}+2 \varepsilon_{1}-V_{f}\left(\varepsilon_{2}-\varepsilon_{1}\right)}
$$

Bruggeman devised a simple model where numerous inclusions can be introduced into a host

$$
\frac{\varepsilon_{c}-\varepsilon_{1}}{\varepsilon_{c}+2 \varepsilon_{1}}=\sum_{i} V_{i} \frac{\varepsilon_{i}-\varepsilon_{1}}{\varepsilon_{i}+2 \varepsilon_{1}}
$$

which was later restructured in the Maxwell-Garnett form [158] [165] [166]

$$
\varepsilon_{c, i}=\varepsilon_{1}+3 V_{f} \varepsilon_{1} \frac{\sum_{i=1}^{N} V_{i} \frac{\varepsilon_{i}-\varepsilon_{1}}{\varepsilon_{i}+2 \varepsilon_{1}}}{1-\sum_{i=1}^{N} V_{i} \frac{\varepsilon_{i}-\varepsilon_{1}}{\varepsilon_{i}+2 \varepsilon_{1}}}
$$

This equation is expanded for two terms, considering the ceramic and porous contributions to the matrix 


$$
\begin{gathered}
\varepsilon_{c p}=\varepsilon_{f 1}+3 \varepsilon_{f 1} \frac{V_{F} \frac{\varepsilon_{f 2}-\varepsilon_{f 1}}{\varepsilon_{f 2}+2 \varepsilon_{f 1}}+V_{p} \frac{\varepsilon_{A i r}-\varepsilon_{f 1}}{\varepsilon_{A i r}+2 \varepsilon_{f 1}}}{1-V_{F} \frac{\varepsilon_{f 2}-\varepsilon_{f 1}}{\varepsilon_{f 2}+2 \varepsilon_{f 1}}+V_{p} \frac{\varepsilon_{A i r}-\varepsilon_{f 1}}{\varepsilon_{A i r}+2 \varepsilon_{f 1}}} \\
V_{F}=\frac{V_{f}}{V_{m}+V_{f}+V_{p}}=\frac{V_{f}}{\left(1-V_{f}\right)+V_{f}+V_{p}}=\frac{V_{f}}{1+V_{p}} .
\end{gathered}
$$

In this equation the permittivity of the matrix and filler must first be modified for stress effects, as is indicated by the subscript $f$. Note that the porous content is represented with the permittivity of air, $\varepsilon_{\text {Air }}$. The filler volume fraction must be considered as part of the entire three phase system, not only as a fraction of the two phase solid solution. This difference is accounted for in (41).

\subsection{Results and Discussion: Application of Model}

\subsubsection{Material Properties}

The complete set of equations used for modeling the thermo-mechanical properties of the composite are displayed in figure 38. Before modeling the sensors, material constants $n$ and $V_{\Delta}$ must be approximated. These constants were empirically found by modeling the response of four sensors from literature [147]. Additional material properties (i.e. thermal, mechanical, dielectric) were found in literature and are displayed in Table 5. The elastic modulus of sensors D and E, which contain a high content of low molecular weight modifier, was approximated considering similar materials in literature [147]. The change in capacitance from an applied load (sensor response) is plotted against the load magnitude in figure 39. In the experimental results, a preload of $40 \mathrm{kPa}$ was used. This is taken into account with the model by using the capacitance at $40 \mathrm{kPa}$ as a reference point for quantifying the change in capacitance. 


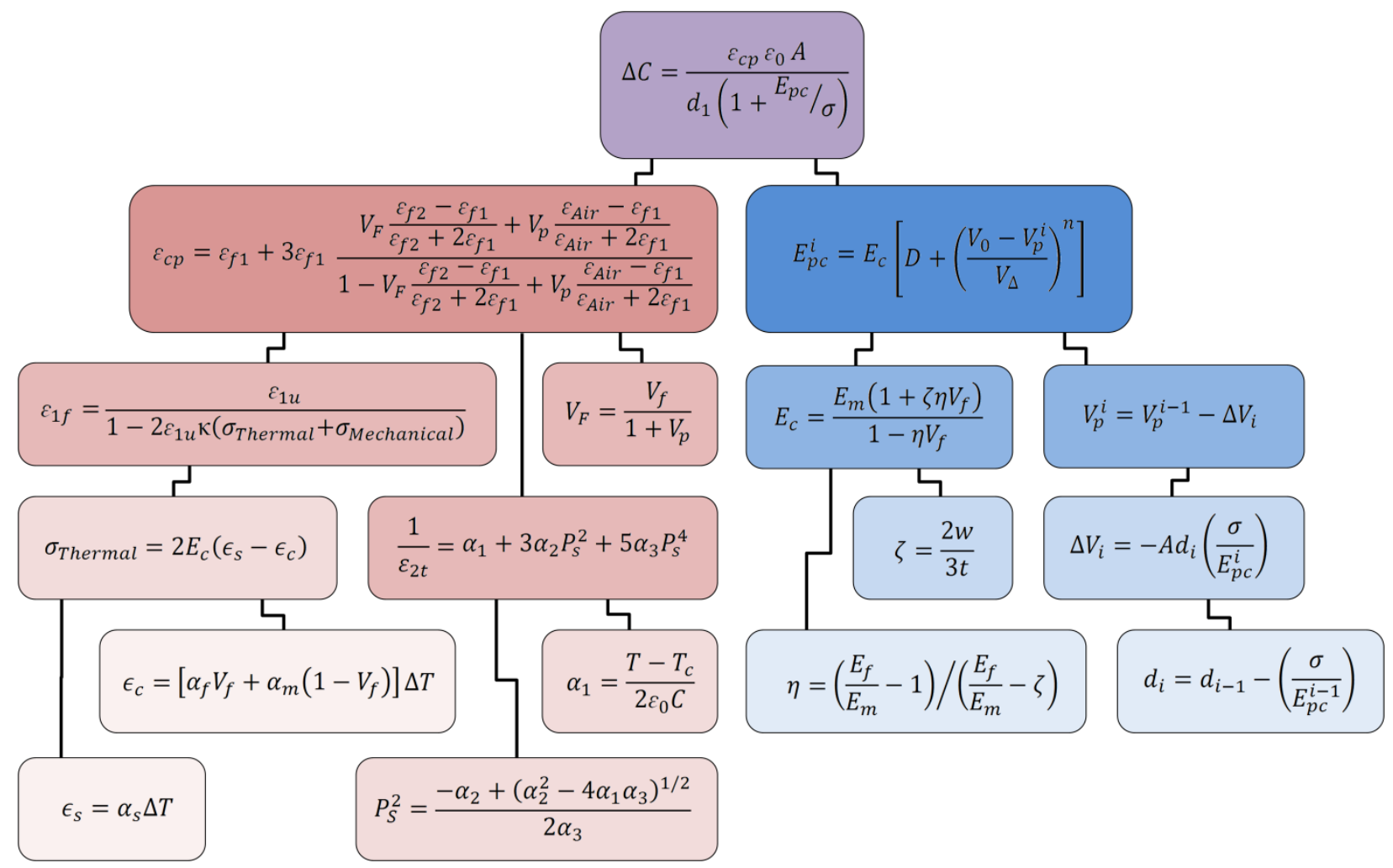

Figure 38. Summary of equations used for modeling the composite sensor.
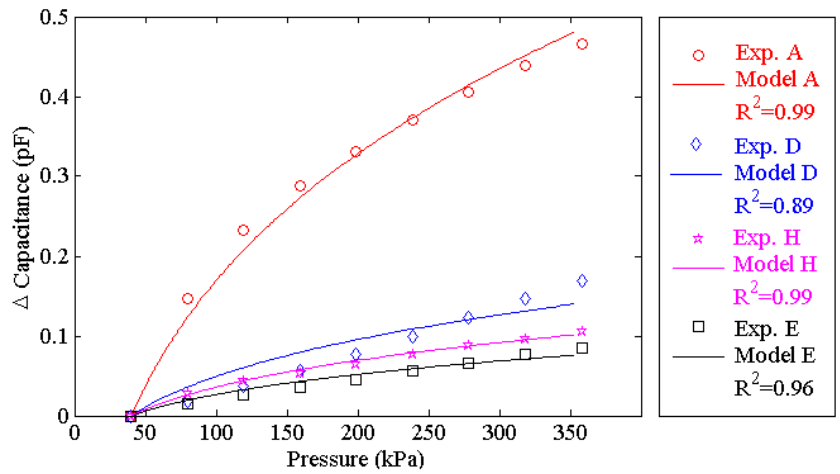

Figure 39. Capacitance change as a function of pressure measured and modeled for various sensor compositions.

In figure 39, sensors were approximated using the analytical equations in this work. Using this model, the role of sensor composition variables can be evaluated for their effect on the character and magnitude of the force response. Experimental results showed that sensor composition A exhibited a much higher capacitive response than other compositions, and thus should be used as a base for future designs [147]. For this reason, all material constants used in the model hereafter will be the same as sensor A, unless otherwise specified for a given section. Using figure 38 as a reverse flow diagram, a program was written to call material variables from Table 5 and perform the iterative load application. 
The proceeding sections evaluate the role of various composite sensor compositions by using a loop to vary specific material constants.

\subsubsection{Effect of Porosity Content}

As noted in section 4.2.2, in figure 37 the character of sensors $\mathrm{A}$ and $\mathrm{H}$, and that of sensors $\mathrm{D}$ and $\mathrm{E}$, were found to be very similar with the exception of $V_{0}$ and $V_{\Delta}$. Since the two variables appear to be interdependent by simple correlation, it is assumed that they are linearly dependent for the sake of performing basic analytical approximations. Using a linear approximation, values for $V_{\Delta}$ were calculated in this section for progressive iterations of $V_{0}$.

The effects of porosity on the capacitive response of the sensor are shown in figure 40. In this figure the amount of porosity is varied from 10-60 vol\%, revealing the effect of porosity on the sensors response (capacitance change) to pressure. The sensitivity of the sensor is exponentially related to porosity as increased porosity slightly reduces the permittivity of the bulk material, and significantly reduces the elastic modulus. Non-linear trends become increasingly apparent with increased porosity. This is caused by increased collapsing of pores at high porosities leading to a more rapidly changing elastic modulus. 


\begin{tabular}{|c|c|c|c|c|c|c|c|}
\hline & Variable & & $\begin{array}{c}\text { Senor } \\
\text { A }\end{array}$ & $\begin{array}{c}\text { Sensor } \\
\text { D }\end{array}$ & $\begin{array}{c}\text { Sensor } \\
\text { E }\end{array}$ & $\begin{array}{c}\text { Sensor } \\
\mathrm{H}\end{array}$ & Source \\
\hline$A$ & Surface Area & $\left(\mathrm{cm}^{2}\right)$ & 0.5 & 0.5 & 0.5 & 0.5 & {$[42]$} \\
\hline$d_{l}$ & Initial Thickness & $(\mu \mathrm{m})$ & 55.8 & 55.6 & 130.5 & 158.6 & {$[42]$} \\
\hline$E_{m}$ & Elastic Modulus of Matrix & $(\mathrm{GPa})$ & 3.44 & 1 & 1 & 3.44 & [79] \\
\hline$E_{f}$ & Elastic Modulus of Filler & $(\mathrm{GPa})$ & 116 & 99 & 116 & 99 & [68-69] \\
\hline$V_{f}$ & Volume Loading of Filler & $(\operatorname{vol} \%)$ & 10 & 20 & 10 & 20 & {$[42]$} \\
\hline$w / t$ & Aspect Ratio of Filler & & 1 & 1 & 1 & 1 & {$[42]$} \\
\hline$V_{0}$ & Initial Porosity & $(\operatorname{vol} \%)$ & 42.5 & 55.6 & 18.1 & 16.6 & [42] \\
\hline$V_{\Delta}$ & Critical Change in Volume & $(\operatorname{vol} \%)$ & 3.7 & 0.47 & 0.46 & 0.9 & a \\
\hline$n$ & Porosity Material Constant & & 3 & 3 & 3 & 3 & $\mathrm{a}$ \\
\hline$\kappa$ & Electrostrictive Constant & $\left(\mathrm{m}^{4} / \mathrm{c}^{2}\right)$ & 0.29 & 0.29 & 0.29 & 0.29 & {$[70]$} \\
\hline$\varepsilon_{1}$ & Relative Permittivity of Matrix & & 6.5 & 6.5 & 6.5 & 6.5 & {$[80]$} \\
\hline$\varepsilon_{\text {Air }}$ & Relative Permittivity of Air & & 1 & 1 & 1 & 1 & [71] \\
\hline$\varepsilon_{0}$ & Permittivity of Free Space & $(\mathrm{F} / \mathrm{m}) \times 10^{-12}$ & 8.854 & 8.854 & 8.854 & 8.854 & \\
\hline$T_{1}$ & Initial Temperature & $\left({ }^{\circ} \mathrm{C}\right)$ & 25 & 25 & 25 & 25 & \\
\hline$T_{2}$ & Final Temperature & $\left({ }^{\circ} \mathrm{C}\right)$ & 25 & 25 & 25 & 25 & \\
\hline$\alpha_{f}$ & Coef. Therm. Exp. for Filler & $\left(\mathrm{m} /{ }^{\circ} \mathrm{Cm}\right) \times 10^{-6}$ & 9.5 & 6.5 & 9.5 & 6.5 & [72] \\
\hline$\alpha_{m}$ & Coef. Therm. Exp. for Matrix & $\left(\mathrm{m} /{ }^{\circ} \mathrm{Cm}\right) \times 10^{-6}$ & 90 & 90 & 90 & 90 & [73] \\
\hline$\alpha_{s}$ & Coef. Therm. Exp. for Substrate & $\left(\mathrm{m} /{ }^{\circ} \mathrm{Cm}\right) \times 10^{-6}$ & 23.4 & 23.4 & 23.4 & 23.4 & {$[74]$} \\
\hline$\sigma_{\text {Mech }}$ & Applied Presssure & $(\mathrm{kPa})$ & 350 & 350 & 350 & 350 & {$[42]$} \\
\hline$\alpha_{2}$ & Dielectric Stiffness Coef. & $\left(\mathrm{m}^{5} /{ }^{\circ} \mathrm{C}^{2} \mathrm{~F}\right) \times 10^{8}$ & -0.7252 & -6.241 & -0.7252 & -6.241 & {$[51,75]$} \\
\hline$\alpha_{3}$ & Dielectric Stiffness Coef. & $\left(\mathrm{m}^{5} /{ }^{\circ} \mathrm{C}^{2} \mathrm{~F}\right) \times 10^{8}$ & 7.5 & 3.23 & 7.5 & 3.23 & {$[51,75]$} \\
\hline$C$ & Curie Constant & $\left({ }^{\circ} \mathrm{C}\right) \times 10^{5}$ & 3.9 & 1.7 & 3.9 & 1.7 & [76-77] \\
\hline$T_{c}$ & Curie Temperature & $\left({ }^{\circ} \mathrm{C}\right)$ & 394.1 & 50 & 394.1 & 50 & {$[77-78]$} \\
\hline
\end{tabular}

aEmperically Determined 


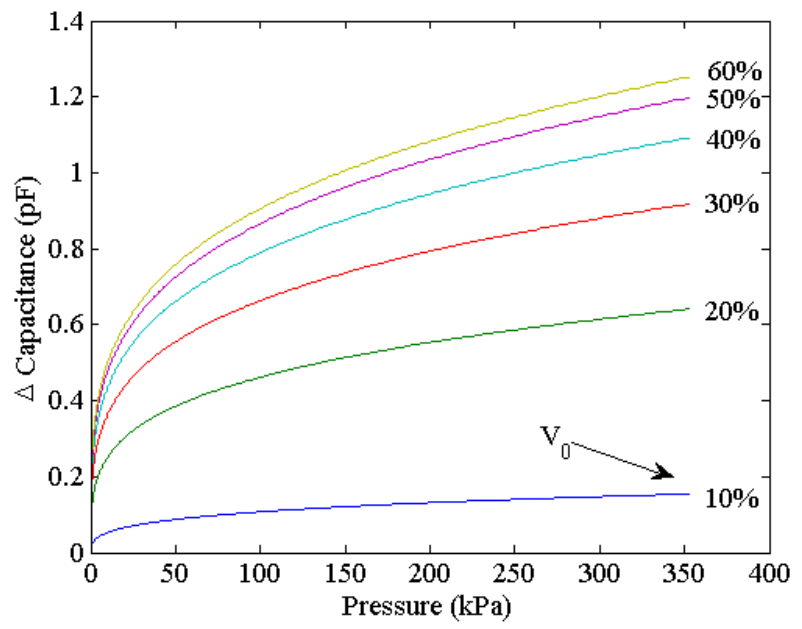

Figure 40. Sensor capacitance change as a function of pressure for sequential porosities, quantifying the effect of porosity on characteristics of the sensor response.

The introduction of non-linearity by increased porosity can be used to further engineer sensors for specific applications. At high porosities, the sensor exhibits highly sensitive attributes at low pressure, with sensitivity decreasing at higher pressure. Porosity can effectually expand the working range of pressure sensors by increasing sensitivity at low pressures, without alterations to the solid solution composition. As the pores collapse under applied pressure, the properties of the solid solution are introduced. A complete model of such behavior will require consideration of plastic effects in the polymer matrix surrounding pores, where increased strain is experienced.

\subsubsection{Effect of Filler Content}

From literature it is known that both the elastic modulus and permittivity of the bulk material exponentially rise with increased filler content $[125,167]$. The inverse relation between these two mechanisms results in a nearly linear rise in the sensor response with increased filler content, as shown in figure 41. The purpose of combining polymers and ceramics in the design of capacitive pressure sensors is to achieve high permittivity and low elastic modulus, optimizing the capacitive response to pressure. If the capacitive response is found to be optimized with either a purely polymeric or ceramic composition, then use of a multi-phase composite is unnecessary. The results in this work indicate that the sensor response is optimized for $V_{f}<100 \%$. However, experimental results in literature show that the response is optimized with a filler volume $V_{f} \approx 10 \%$ [147]. The difference is believed to be a function of the filler influence on the elastic modulus $E_{p c}$. In order to more accurately model the effect of the filler content on the porous composite, addition experimental data is needed to quantify trends. 


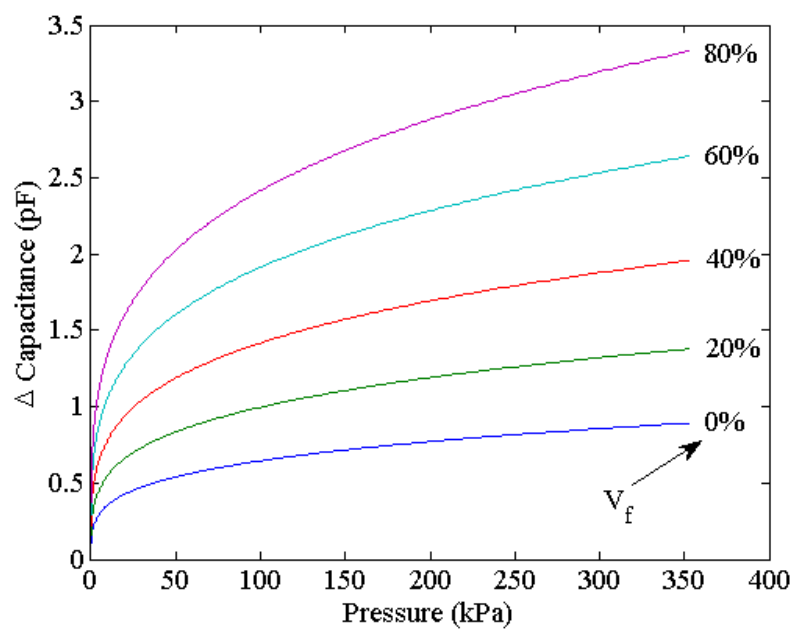

Figure 41. Sensor capacitance change as a function of pressure for sequential filler contents, quantifying the effect of filler loading on characteristics of the sensor response.

\subsubsection{Effect of Temperature}

Thermal effects on the composite sensor were evaluated by considering the changes in permittivity of the matrix and filler. Using (32), changes in the permittivity caused by electrostriction are calculated as a function of thermal stress, assuming the sensor was cast onto a steel substrate with a curing temperature of $25{ }^{\circ} \mathrm{C}$. The permittivity of the filler was approximated as a function of temperature using (33). The permittivity of the matrix and filler are plotted independently as a function of temperature in figure 42 . Since increased temperature causes permittivity to decrease in the matrix and increase in the filler, the effect of temperature on the bulk permittivity is dependent on the filler volume loading. Thermal effects in the matrix were found dominant until the filler volume approached $V_{f}=7.3 \%$. 


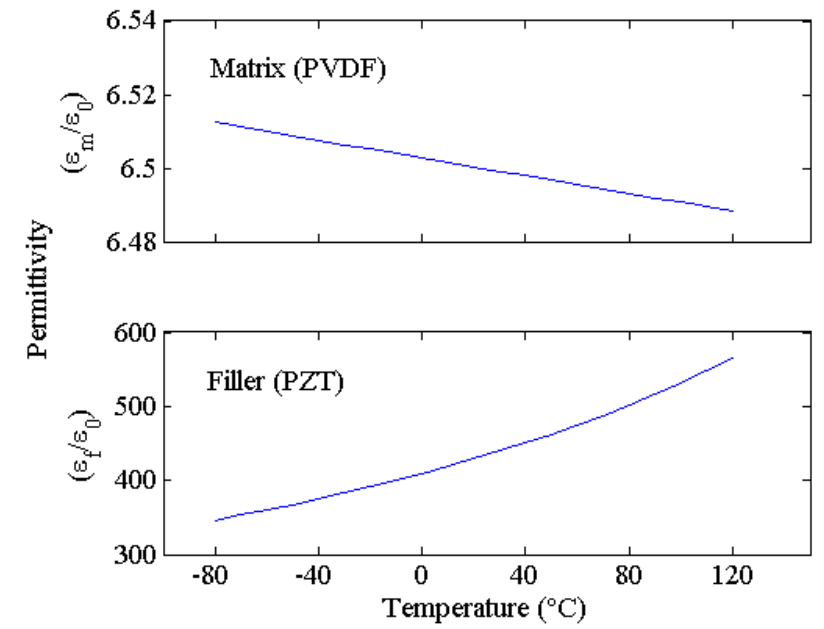

Figure 42. The permittivity of the matrix and filler constituents plotted as a function of temperature.

The change in capacitance is plotted as a function of temperature in figure 43 for both the model and the experimental data. Experimental data was recorded with a pressure of $40 \mathrm{kPa}$, thus the model also used this pressure for the approximated capacitance.

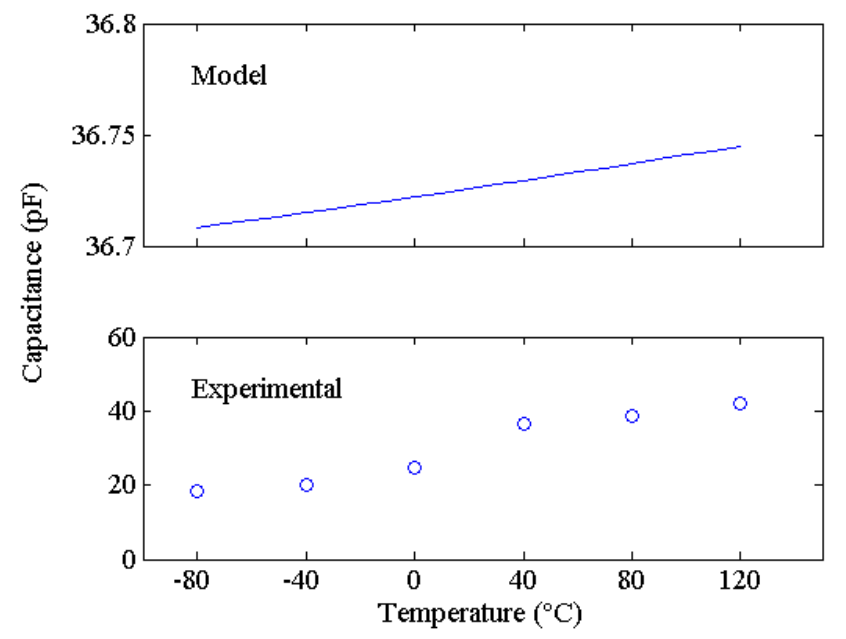

Figure 43. Modeled and experimental capacitance change of the three-phase sensor as a function of temperature.

The thermal effects approximated in the model are negligible as compared with experimental results, showing the need for additional work in this area. Since modeled thermal effects in the matrix permittivity are minimal, and the matrix permittivity has the largest influence in the bulk permittivity, it is believed the source of error occurs in the matrix approximations. More specifically, the assumption of a fully paraelectric phase, amorphous, polymer matrix is believed to be the cause of this error. The semi- 
crystalline nature of PVDF can be difficult to model both mechanically and electrically [168] [169]. Since crystalline PVDF has a Curie temperature in the range of $100{ }^{\circ} \mathrm{C}$, and even when combined with surfactants or copolymers, thermally induced polarization would see a large rise in permittivity in this range $[170,171]$. The spike may not be apparent in the experimental data because of pining by the substrate, which is known to cause diffusivity in the dielectric properties, and shifting of the Curie temperature [172].

\subsection{Conclusions}

This work successfully laid the foundation for future work pertaining to modeling the capacitive response of three-phase, polymer-matrix pressure sensors. Using relations between capacitance, permittivity, and elastic modulus, a model was developed to predict capacitance as a function of pressure. The elastic modulus and permittivity were approximated for the three-phase system, and substrate and thermal effects on permittivity were taken into account. The final model requires an iterative application, resulting from an interdependency of the elastic modulus and porosity. Material constants required for modeling the porous elastic modulus were approximated by empirically fitting experimental data from literature.

The model was applied to evaluate composition and thermal effects on the response. The capacitive response was increased exponentially with the introduction of porosity. Porosity also induced non-linear trends in the sensor response. Additional ceramic filler content increased the sensor response for $V_{f}<100 \%$, although experimental data in literature shows that the response is optimized at $V_{f} \approx 10 \%$. Thermal effects were found to be dependent on the filler volume, as the polymer matrix experienced lower permittivity with increased temperature and the ceramic filler experienced higher permittivity. Additional work is needed to approximate the thermal effects of the matrix, which may contain crystalline domains. 


\section{Chapter 5. Capacitive Force/Spatial Awareness Sensor for Robotic Space Servicing Applications}

\subsection{Introduction}

The goal of this work is to design and fabricate flexible, capacitive force sensors based on a 3:0:3 porous ceramic-polymer composite design for use in harsh environments, specifically space servicing robotic applications. Selection of materials and processing methods will be carried out accordingly, providing a robust sensor fabrication sequence resulting in application ready specimens. Preliminary work was performed in chapters 3 and 4, and will be continued herein by applying these previous works.

There are a number of variables to be considered in optimizing sensor properties, including sensor thickness, ceramic composition/particle size/volume content, molecular weight and molecular architecture of polymer and modifier components, polymer/modifier ratio, and porosity content. Furthermore, the scope of this work includes the design and implementation of characterization procedures necessary for qualifying the resulting material compositions for use in in-orbit robotic operations. Evaluation criteria includes electrical, mechanical, and thermal properties affected by phase transitions and microstructure variables.

\subsection{Experimental}

\subsubsection{Sensor Design}

A three phase, porous polymer-ceramic composite was used to create a low modulus, high dielectric sensor composition. Specific parameters for optimization of the sensor properties were obtained through previous experimental and analytical studies performed in chapters 3 and 4 . The primary parameters outlined in previous works are outlined here with the intent to optimize composition variables. The addition of ceramic filler materials was found to exponentially increase the elastic modulus, while at the same time increasing the dielectric constant. Experimental testing demonstrated that the sensor response was optimized with a ceramic filler loading below 20 vol $\%$ of the solid loading (excluding consideration of porosity). In this work, a filler loading of $10 \mathrm{vol} \%$ was used. Analytical modeling indicated that the sensor response was most significantly increased using a porosity of 20 vol\%. Lesser amounts of porosity did not significantly increase the sensor response from the non-porous response. Similarly, increased porosity less significantly contributed to the sensor response, meaning that doubling the porosity content did not double its contribution to the response. For this reason, the target 
porosity was $20 \mathrm{vol} \%$ of the three phase composite, limited to decrease plastic deformation expected from large strain in the polymer matrix.

Considerations were made for use of a conductive metallic or carbon phase in the composite sensor composition. Use of various conductive particles in dielectric materials has been proposed in literature, as implementation of conductive filler just below the electrical current percolation threshold increases permittivity of the bulk material [173]. This approach could further increase the permittivity of the sensor material, in effect increasing the response of the sensor. However, introduction of increased high dielectric filler particles is already limited by the need to maintain a low elastic modulus and the addition of conductive filler particles would further increase the modulus if added in addition to the dielectric filler. The conductive filler could also be used to replace a portion of the ceramic filler content, preventing alteration of the elastic modulus, but the result would reduce the permittivity of the bulk material and countering their intended purpose. Additionally, the incorporation of conductive filler materials would significantly affect the breakdown voltage, resulting in an increase in the required film thickness. Reduction of the film thickness is already limited by the reduction of the breakdown voltage, and reduced thickness is necessary to increase the capacitance and simplify embedding of the sensor for robotic applications. For these reasons use of conductive filler materials, while seemingly valuable, was not recommended in this work.

The polymer matrix was selected for having a low elastic modulus, allowing increased strain in the sensor. Because it was the most prevalent material in the three phase composite, a high dielectric constant was also important. A high molecular weight and complex molecular architecture were desired to restrict movement of the filler material. It was also advantageous to select a material lacking any major phase transformations in the working temperature range, which provides a consistent elastic modulus under the thermal loading. The polymer matrix must also meet the ASTM E595 outgassing standards, preventing outgassing upon entering low vacuum, high temperature environments. The selection of controlled volatility polymers includes considerations such as molecular weight, molecular weight distribution (polydispersity), functional groups, and appropriate bond accepters for solvents [174]. Another consideration is the potential use of polymer modifiers, which display a low molecular weight and are unable to cross-link with the prime polymer; these modifiers could potentially outgas when introduced into a high vacuum or high temperature environment. Additional conditioning could be performed during the manufacturing of the polymers to remove such modifiers, or low-outgassing materials can be used to reduce pre-processing of the CV materials [175]. Because of their adverse effects on outgassing properties, organic modifiers in the polymer matrix were removed.

With these considerations use of a cross-linking polyurethane was prosed, as military specifications (MIL-SPEC) have been based on these materials for rigorous military applications on land 
and in space. Specifically, the mechanical, thermal, and electrical properties of two part Uralane 5750 and 5753 have been deliberately and extensively tailored to meet the needs of applications such as those proposed in this work. Such considerations included the molecular weight, molecular architecture, crosslinking density, and ratio of long polyol chains and polyurethane/polyuria-based linkages making up the two part Uralane [176]. Ultimately Arathane 5750 (Huntsman, $1.05 \mathrm{~g} / \mathrm{ml}$ ) was selected, being a crosslinking thermoset polymer with a dielectric constant of 3.0, a Shore A hardness of 50, and meeting outgassing standards.

Two unique sensor composites were fabricated, differing only by the composition of the filler material. The first composition contained $\mathrm{BaTiO}_{3}$ (BTO, Inframat, Manchester, CT) filler. This ceramic was selected because BTO is available from industry, and BTO thin-films have shown a high dielectric constant (above 300) and high breakdown strength (as high as $2.5 \times 10^{6} \mathrm{~V} / \mathrm{cm}$ ) [177]. The Curie temperature of BTO is near the desired working range $\left(130^{\circ} \mathrm{C}\right)$, known to introduce undesirable thermal effects on the sensor response, as well as additional phase transitions below the Curie temperature. The particle size of the filler was minimized to promote electric flux percolation within the composite without increasing the filler content, and to reduce the filler's contribution to the elastic modulus [178-179]. Reduction of the particle size was limited by the ferroelectric size effect, which was found to reduce the Curie temperature for particles smaller than $80 \mathrm{~nm}$ [180]. For this reason a particle size of $100 \mathrm{~nm}$ was used for BTO.

The second sensor composite used $\mathrm{Pb}\left(\mathrm{Zr}_{0.44}, \mathrm{Ti}_{0.56}\right) \mathrm{O}_{3}(\mathrm{PZT})$ as the filler composition, which was desired because of its high dielectric constant. This composition was also preferred since its Curie temperature and other phase transformations were far outside the working temperature range, preventing the introduction of thermal effects on the sensor response. Reduction of the particle size was limited by the ferroelectric size effect, which was found to shift the Curie temperature into the working temperature ranges for particles of $30 \mathrm{~nm}$ or smaller for $\mathrm{PbTiO}_{3}$ [180-181]. Assuming a Gaussian distribution in the particles size, the target mean particle size was $100 \mathrm{~nm}$, which minimizes the content of particles smaller than $30 \mathrm{~nm}$. The chosen particle morphology was spherical in shape, since particles with an increased aspect ratio may result in oriented microstructures during processing. The oriented microstructures result in varied distribution in properties depending upon the degree of orientation, and this phenomena is not of interest in this work. The primary concern was changes in the senor response caused over time (sensor drift) due to possible reorientation of these particles during force applications.

The specific PZT composition and size criteria desired for this work were not readily available by commercial vendors. For this reason, the PZT powder was synthesized using a modified hydrothermal process based on the work by Deng et al. [182]. Using a $150 \mathrm{ml}$ Teflon autoclave liner, $95 \mathrm{ml}$ of deionized (DI) water was added and stirred with a magnetic stirring rod. A weight of $4.51 \mathrm{~g} \mathrm{ZrOCl}{ }_{2} \bullet 8 \mathrm{H}_{2} \mathrm{O}$ 
(Alfa Aesar), and $6.62 \mathrm{~g} \mathrm{~Pb}\left(\mathrm{NO}_{3}\right)_{2}$ (Alfa Aesar) were added and stirred for 30 minutes. Since a minimum particle size of $30 \mathrm{~nm}$ was desired, $0.767 \mathrm{~g}$ nano- $\mathrm{TiO}_{2}(\sim 32 \mathrm{~nm}$ size, Alfa Aesar) particles were used as seeds for the process. Concurrently, $33 \mathrm{~g}$ of potassium hydroxide $(85 \% \mathrm{KOH}$, Alf aAesar) was added to the solution which resulted in a $\mathrm{pH}$ of 14.2 , and the solution was stirred for one minute. The solution was sealed and the reaction was completed at $260{ }^{\circ} \mathrm{C}$ with a hearting rate of $2{ }^{\circ} \mathrm{C} / \mathrm{min}$. The reaction temperature was maintained for $3 \mathrm{~h}$; after the reaction was completed, the reactor was cooled to room temperature at a rate of $2{ }^{\circ} \mathrm{C} / \mathrm{min}$.

The resulting PZT dispersion was electrostatically dispersed by the high $\mathrm{pH}$ in the $\mathrm{KOH}$ solution. During the first rinsing cycle, a centrifuge speed of 1,200 rpm was utilized for 10 min which resulted in the sedimentation of only the larger particles. The fine nanoparticles remained within the supernate, therefore allowing for efficient separation and disposal of particles below $50 \mathrm{~nm}$, which could introduce the Curie temperature into the working range by the ferroelectric size effect. In order to maintain the colloidal dispersion, a $\mathrm{pH}$ greater than 10 was required [183]. The remaining $\mathrm{KOH}$ was rinsed from the particles using a mixture of $25 \mathrm{ml}$ DI water and $1 \mathrm{ml}$ tetramethylammonium hydroxide (TMAH, AlfaAesar). This process was carried out by mixing the new solution with a magnetic stirrer and a Sonics Vibra-cell sonication wand at $50 \%$ power for $1 \mathrm{~h}$. The dispersed suspension was then centrifuged again at 2,500 rpm for $15 \mathrm{~min}$. The supernate was again separated from the precipitated PZT and the process was repeated on the precipitated powder (and the supernate was discarded). The solution was carefully watched to prevent elevated temperatures. Using the TMAH solution, this method was repeated for a total of 5 times to remove all $\mathrm{KOH}$.

The polymer selected for the matrix required xylenes as a solvent to maintain its controlled volatility. Since the PZT filler was synthesized and dispersed in an aqueous solution, it was necessary to undergo a solvent exchange from the aqueous solution to xylenes. Large differences in polarity between water and xylenes prevented a simple emulsion, so the polarity was stepped down in stages. With the same stirring, sonication, and centrifuge methods previously described, the PZT powder was rinsed in 6 cycles, where the polarity of the solvent was slowly changed from polar to non-polar over the number of cycles. The solvent in each cycle were as follows: (1) $50 \mathrm{ml}$ of ethanol, (2) $25 \mathrm{ml}$ ethanol/25 ml 1butanol, (3) $50 \mathrm{ml} \mathrm{1-butanol,} \mathrm{and} \mathrm{(4-6)} 50 \mathrm{ml}$ xylenes. During the exchange, one drop of oleic acid was added to each solution prior to rinsing the PZT, acting as a surfactant to promote electrosteric dispersion of the nano-particles upon removal from the aqueous solution. The wetting of the PZT particles was controlled by the oleic acid by providing the functional carboxyl head to be attracted to the PZT surface while the hydrocarbon tail has an affinity to the xylenes solvent.

Failure to properly remove the $\mathrm{KOH}$ from the surface of the particles was found to prevent proper dispersion of the particles in the polymer matrix, and resulted in a reduction in strength and modulus of 
the composite by inhibiting bonding between the PZT and polymer matrix. Also, in the case that the 1butanol was not completely removed from the dispersion, the solvent inhibited the polymer from properly hardening (acted as a polymer modifier), which left a soft and tacky film after the drying process. The work also showed that any excess oleic acid was removed during the centrifuge process, but any excess surfactant did not notably affect the properties of the resulting thin film other than introducing an orange color in high concentration.

The crystal structure of the synthesize PZT powder was evaluated using X-ray diffraction (XRD). The diffraction pattern is shown in figure 44. Automated Rietveld refinement of the XRD pattern was utilized in X'Pert Highscore software to characterize the final phase content (Zr distribution) within the powder. The analysis showed that $\sim 77 \%$ of the material was zirconium-rich PZT (rhombohedral, JCPDS 01-089-1276), and $\sim 23 \%$ was titanium-rich (tetragonal, JCPDS 00-050-0346). The PZT phase diagrams at room temperatures show a phase shift at $52 \%$ titanium $/ 48 \%$ zirconium, where lesser titanium results in the rhombohedral phase and more titanium results in tetragonal phase [184]. The broad peaks in figure 44 indicate nanometer-size particles with some variance in the crystal phase. If the $\mathrm{Ti} / \mathrm{Zr}$ ratio varied as little as $2 \%$ throughout the seeds, then an additional phase was expected. For this reason, the XRD results were not unexpected, and indicate successful synthesis of a PZT composition similar to the desired $\mathrm{Pb}\left(\mathrm{Zr}_{0.44}, \mathrm{Ti}_{0.56}\right) \mathrm{O}_{3}$. However, further characterization is required to more fully characterize the exact composition.

The particle size was determined using dynamic light scattering method, where the particle size distribution was calculated from the average of 12 runs. The particle size is plotted against the number of particles, showing a Gaussian distribution in figure 45. The particle shape was observed using a scanning electron microscope (SEM). Figure 46 shows a SEM micrograph of the PZT nano-particles, having the desired aspect ratio near 1 (spherical shape). The particles were sputtered with gold to create a conductive surface for electron scanning. Vacuum deposited gold is known to produce a pebble-type morphology, which can be seen in the images with a diameter $\sim 10 \mathrm{~nm}$ [185]. Fully visible PZT particles range from 100-150 $\mathrm{nm}$ in diameter. The BTO particles were characterized by the manufacturer using SEM and Brunauer-Emmett-Teller (BET), with a reported particle size of $100 \mathrm{~nm}$. The BTO particles are also shown in figure 46 , showing the similarity in aspect ratio and particle size between the two ceramics. 


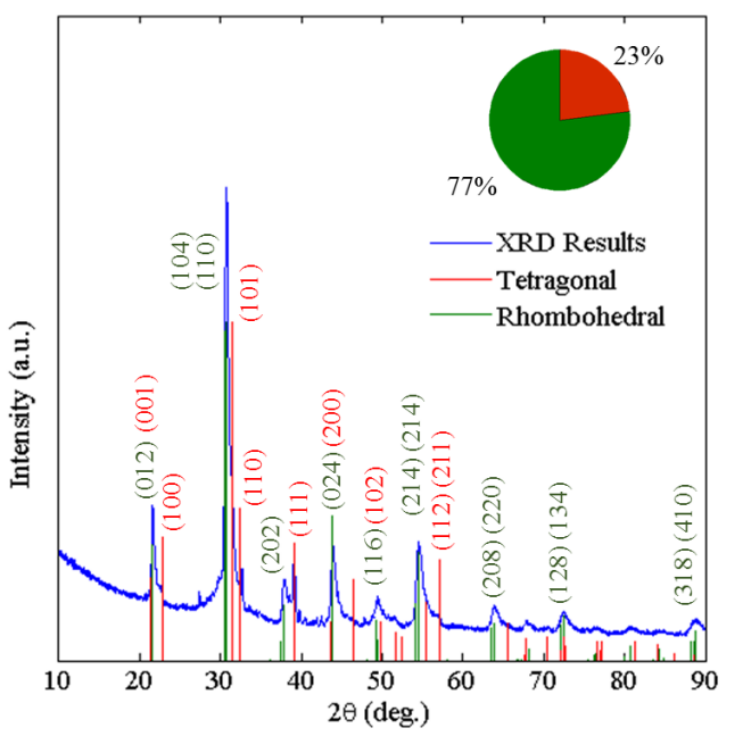

Figure 44. XRD analysis of synthesized PZT, showing estimated rhombohedral (zirconium rich) and tetragonal (titanium rich) phase distribution.

Size Distribution by Humber

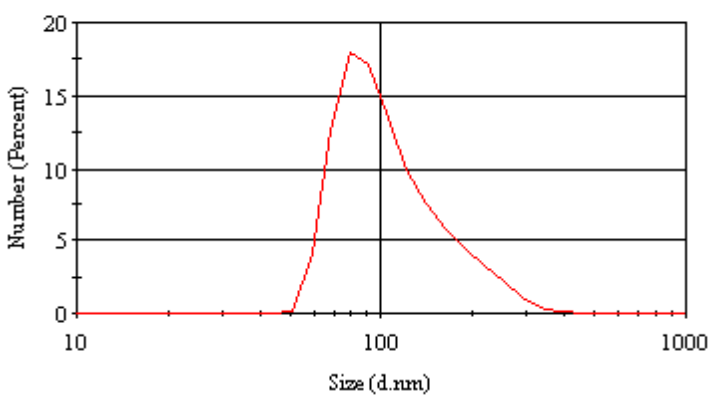

Figure 45. Particle size distribution of synthesized PZT obtained by dynamic light scattering, plotted against the number of particles.

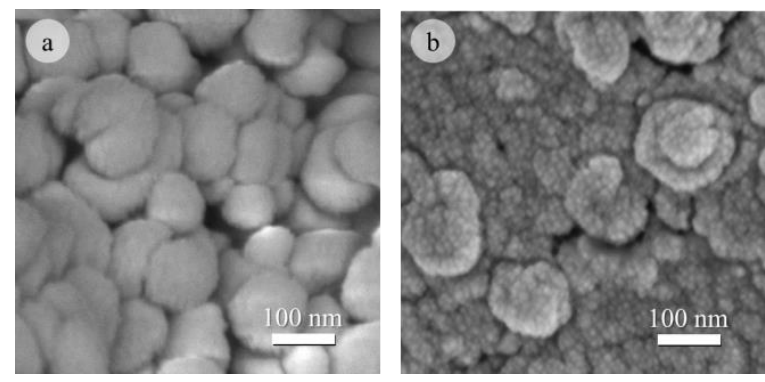

Figure 46. SEM micrograph of a) purchased BTO, and b) synthesized PZT, showing size and aspect ratio of particles. 


\subsubsection{Sensor Fabrication}

The fabrication of sensors was initiated by creating a sensor ink for each of the two compositions. The inks were composed of 66.66 vol\% solids, and 33.33 vol\% solvent. The solid loading was divided into 90 vol\% Arathane 5750 (Huntsman, $1.05 \mathrm{~g} / \mathrm{ml}$ ) polymer and $10 \mathrm{vol} \%$ ceramic filler. The only solvent used was xylenes. The preparation of the BTO/polymer composite solution began by dispersing the dry BTO powder in xylenes using a sonication wand at $50 \%$ power for $1 \mathrm{~min}$.

The PZT/xylenes composition was prepared by first removing excess xylenes using a centrifuge at 2,500 rpm for $15 \mathrm{~min}$. After pouring off the xylenes from the precipitated PZT, a sample of the solution was taken using a metal spatula. A small piece of metal foil was placed on a high precision scale, and the scale was zeroed with the foil. The sample was then weighed on the foil, dried on a hot plate, and weighed again to determine the solid loading of the PZT/xylenes solution. By dividing the final weight over the initial weight, a solid loading of approximately $85 \mathrm{wt} \%$ was typically found.

Using a small glass container to prevent melting from the sonication wand or solvents, $0.2152 \mathrm{~g}$ of PZT was measured taking into account the weight loading of the solution. Additional xylenes was added as needed, bringing the total xylenes content to $0.1208 \mathrm{~g}$, and a sonication wand was used at $30 \%$ to break up PZT agglomerations that were formed in the centrifuge. The Arathane 5750 thermoset requires two parts for polymerization, the cross-linking agent $(0.0400 \mathrm{~g}$ part $\mathrm{A})$ and the monomer $(0.2225$ g part B). After combining all materials, a sonication wand was used to disperse the nanoparticles throughout the polymer. The composite was then stirred using a magnetic stirring rod at the highest speed for one minute, ensuring homogenous distribution of the constituents.

A polished glass base was prepared with a sheet of aluminum foil, which was the substrate for the sensor composition. The foil was cleaned using xylenes to remove oils and dirt. Using a metal spatula, the sensor ink was deposited as a thin line across the aluminum foil at an approximate width of $8 \mathrm{~cm}$. A mayer rod (\#4, R.D. Specialty, Webster, NY) with a casting thickness of $9.5 \mu \mathrm{m}$ was then used to cast the composition across the face of the foil. A mayer rod is a wire wound rod, which deposits a film when pulled (without spinning) over a bead of viscous material across a substrate. The casted material was immediately placed in a vacuum oven at $125^{\circ} \mathrm{C}$ and vacuum of $15 \mathrm{in}-\mathrm{Hg}$. The desired porosity of 20 vol\% was targeted by quickly removing the solvent to leave void space (pores), and having a small amount of excess solvent (13 vol\%) to adjust for shrinking of the film thickness (which collapses void spaces). The drying process was maintained for $12 \mathrm{~h}$ in order to insure that all xylenes was removed to maintain the controlled volatility of the polymer. The final sensors were prepared by using a metal punch to prepare $9 \mathrm{~mm}$ diameter specimens from the bulk film. The use of spin coating and tape casting with a doctor blade were also evaluated for deposition of the thin-film. The spin coating technique required a 
dispersion with a low solids loading (25 vol\%) in order to reduce the viscosity to a level that would achieve the desired minute thickness. The low viscosity dispersion resulted in the phase separation by settling which produced an inhomogeneous final film. Tape casting with a doctor blade produced a homogenous film composition, but the film thickness varied because the aluminum substrate was not perfectly flat.

A scanning electron microscope was used to examine the final film thickness and microstructure. The top face of the specimens was prepared with $10 \mathrm{~nm}$ of gold that was deposited by DC sputtering. Precautions were taken in order to prevent damage or contamination of the specimens during the cutting of the cross-sectional samples. Two pieces of $200 \mu \mathrm{m}$ thick microscope cover-glass (minimized to reduce SEM charging) were lightly coated with EpoxyBond 110 two part epoxy. The specimens were placed between the two microscope cover-glass slides and the glass slides were pressed together. The sandwiched samples were heated to $160{ }^{\circ} \mathrm{C}$ for $5 \mathrm{~min}$ to cure the adhesive. A glass slide was used as a substrate for cutting, and the prepared specimens were bounded using wax. The glass slide was then installed in a diamond blade saw, and the specimens were cut as shown in figure 47 . The diced specimens were cleaned with acetone, removing wax and lubricating oil, and their cross sections were sputtered with gold.

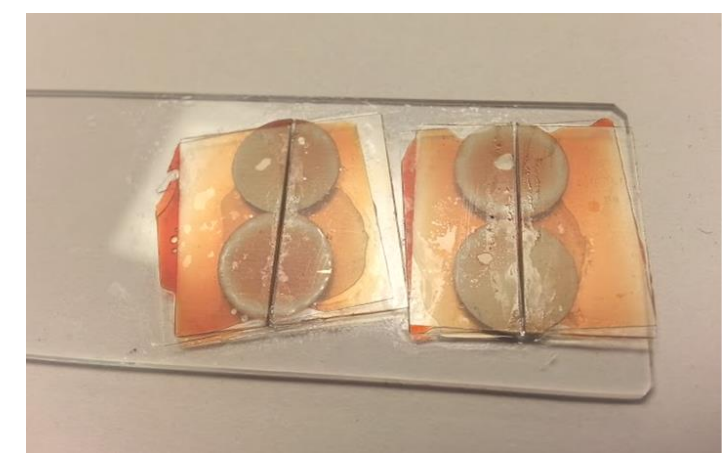

Figure 47. Preparation of specimens for cross-sectional observation using SEM.

The cross-sectional SEM micrograph in figure 48a confirmed the successful casting of the thin film with an approximate thickness of $8.5 \mu \mathrm{m}$. The figure also shows successful bonding of the film onto the aluminum substrate, without the presence of notable cracking or separation between the layers. The microstructure of the top surface of the film is shown in figure 48b. It appears that submicron pores were collapsed by the vacuum treatment during the drying process. The collapsed pores displayed an increased aspect ratio and resembled cracks rather than pores. Regardless of the shape, these void spaces are prevalent and large in size relative to the thickness of the film. The final cured thickness of the specimen 
was reduced by approximately $10 \%$, indicating a final porosity level of $\sim 23 \mathrm{vol} \%$, considering the initial solid loading of $66 \mathrm{vol} \%$.
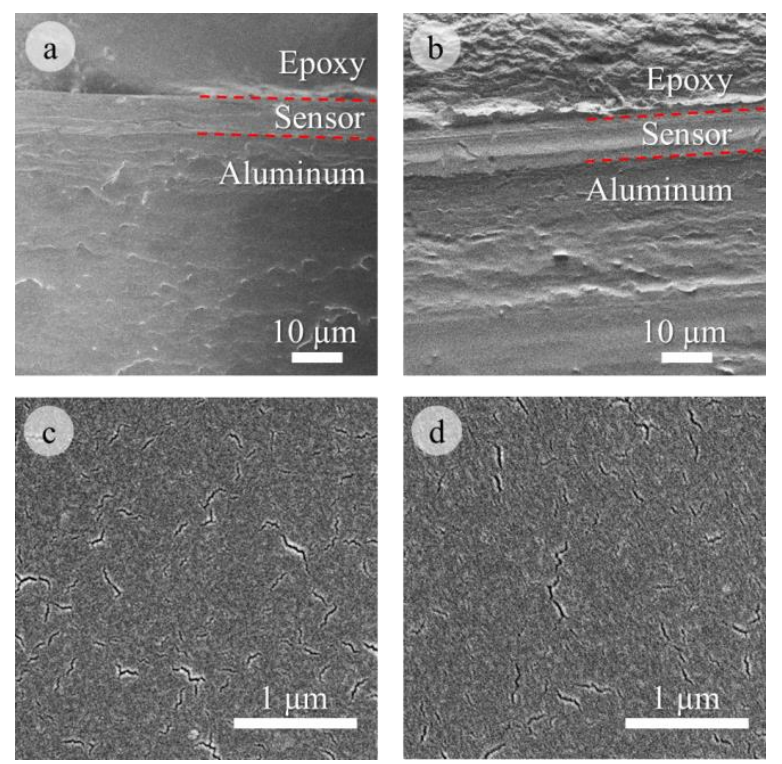

Figure 48. SEM micrograph of thin-film cross section for (a) BTO filled composition and (b) PZT filled composition, and top surface of (c) BTO filled composition and (d) PZT filled composition.

\subsection{Sensor Characterization}

Thermal and mechanical loading was applied using a TA Instruments DMA Q800. While loading, in-situ electrical acquisition was carried out using a BK Precision 889B LCR meter by applying $1 \mathrm{~V}$ with an AC frequency of $200 \mathrm{~Hz}$ and recording the capacitance and dielectric loss values. A thermally resilient silicone rubber was adhered to the active arm of the DMA to create a mechanically compliant layer, in an effort to ensure homogenous distribution of the compressive stress across the sensor face. Mechanical loading and electrical acquisition were performed across an $8 \mathrm{~mm}$ diameter, preventing unintentional contact around the $9 \mathrm{~mm}$ diameter specimen which would result in a short circuit.

The surface of the silicone on the active DMA clamp, and the surface of the static DMA clamp were both prepared with copper foil, working as electrodes in contact with the sensor faces. The electrical leads from the LCR meter were passed through the vent of the DMA chamber, and bridged to the copper contacts with silver micro-wire. The flexible micro-wire allowed for electrical acquisition without interfering with the application of the mechanical load. The full testing setup is shown in figure 49. For each sensor composition, a total of 5 specimens were prepared. These same 5 specimens were used for every test, showing compounding degradation of the materials through various loading 
schedules. All tests were performed in the order which they are introduced in this section, which is: thermomechanical $\rightarrow$ room temperature $\rightarrow$ cyclic $\rightarrow$ long term loading.

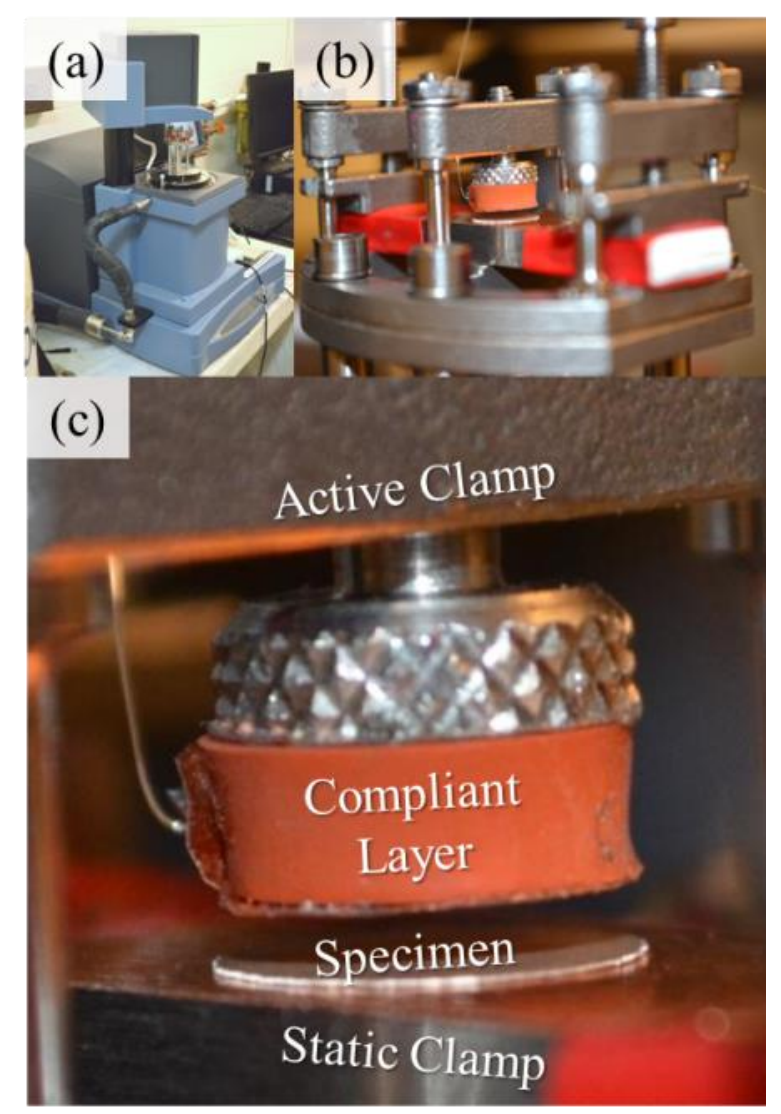

Figure 49. Thermomechanical testing setup: a) DMA instrument, b) grip configuration for testing with in situ electrical acquisition, and c) compliant layer used to ensure homogenous pressure distribution.

Thermomechanical testing was performed from $120{ }^{\circ} \mathrm{C}$ to $-80{ }^{\circ} \mathrm{C}$, where the mechanical load and electrical data acquisition was completed at every $40{ }^{\circ} \mathrm{C}$ increment. The high temperature testing was completed through the use of heating coils built into the DMA chamber hood. Low temperatures were acquired using liquid nitrogen that was injected into the thermal hood by the automated DMA system. Mechanical testing of the specimens was not initiated until the chamber gained equilibrium of the desired temperature which typically took $8 \mathrm{~min}$. Precautions were taken to prevent excessive damage to the electrical leads during opening and closing of the chamber hood at extreme temperatures. At high temperatures, the plastic insulation surrounding the LCR leads was found to melt, and at low temperatures the insulation was found to crack and shatter. In order to prolong the lifetime of the LCR leads, the leads must be carefully secured to prevent contact with the heating coils and the system must be returned to atmospheric conditions before opening the DMA hood. 
At each temperature, the stress was cycled 10 times in steps from $20 \mathrm{kPa}$ to $360 \mathrm{kPa}$ at a rate of 12 seconds per cycle. A total of 5 specimens were tested for each composition. These results were intended to quantify the thermal effects on the magnitude of the sensor response. One area of concern was the extent of thermal drift, undesired change in magnitude under thermal stress, due to alterations in the composite microstructure. The purpose of using a step load, rather than a ramp load, was to check for a rate dependant hysteresis that can be introduced by thermal effects on the polymer matrix.

Room temperature mechanical testing was performed using a preload of $20 \mathrm{kPa}$, a loading rate of $120 \mathrm{kPa} / \mathrm{min}$, and a maximum stress of $360 \mathrm{kPa}$. A total of 5 specimens were characterized for each sensor composition, with 10 loading ramps performed on each specimen. The purpose of this testing was to define the trend of the sensor response, which is the change in capacitance under application of a quasistatic loading schedule. It was also meant to gain an understanding of changes in the composite composition as initial mechanical loading caused settling in the microstructure.

Cyclic loading was applied to sensors across the entire $9 \mathrm{~mm}$ diameter of the specimens to ensure uniform degradation of the entire sensor microstructure. Loading was performed at $1 \mathrm{~Hz}$, stepping the stress from $16 \mathrm{kPa}$ to $280 \mathrm{kPa}$. A total of 100,000 cycles were applied to each of the 5 specimens. Using a stacked approach, all 5 specimens were loaded simultaneously. To prevent stress concentrations of the applied load, a small compliant layer was placed between each specimen during loading as shown in figure 50. After completing 100,000 cycles, specimens were individually characterized to show the effects on the character of the sensor response. This was achieved by performing an additional 10 loading cycles to ensure contact of the electrodes, then applying a ramp load using the same procedure described for the room temperature characterization.

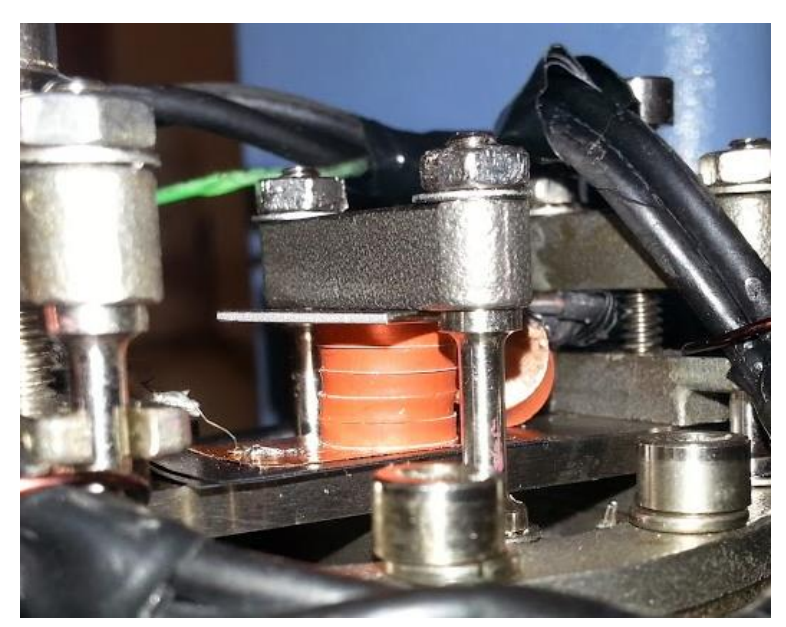

Figure 50. DMA configuration for simultaneous cyclic loading of 5 specimens, using silicone to distribute stress across the sensor face. 
The creep loading was applied at $360 \mathrm{kPa}$ for $24 \mathrm{hrs}$. The electrical acquisition was performed throughout the entire loading cycle to investigate the drift of the sensor response. In an effort to ensure constant load application, a static weight was used for this testing. The active arm applying the load was prepared in exactly the same manner as the active arm of the DMA. Sample preparation and electrical acquisition were also precisely the same.

Outgassing of the final composition was quantified using ASTM E595 as an outline. For each composition, 5 specimens having a $15 \mathrm{~mm}$ diameter were collected and weighed on a high precision scale. Clean aluminum foil substrates were also weighed, and used as a reference for the experiment. All samples were left in atmospheric condition for a minimum of $24 \mathrm{hrs}$ after processes. This gives the porous materials an opportunity to absorb moisture from the air. After weighing the specimens they were placed on a clean glass petri dish and moved into a vacuum of $3 \times 10^{-6}$ torr. After achieving the full magnitude of the vacuum, the specimens were left for $24 \mathrm{hrs}$. Upon removal from the vacuum, specimens were immediately weighed. All specimens were weighed within 2 min of being removed from the chamber, preventing the materials from re-absorbing moisture from the air. Because of the strict time restraints, all 5 specimens of each composition were weighed together at one time.

\subsection{Results and Discussion}

The thermomechanical loading results were summarized by showing the last cycle of the 10 mechanical loading cycles performed for each thermal load. All five specimens were plotted for each filler composition, giving a summary of the composition properties throughout the loading schedule. Shown in figures 51 and 52, the capacitive response to the mechanical loading cycle ( $20 \mathrm{kPa}$ to $360 \mathrm{kPa}$ ) is plotted against the six thermal loading steps. The magnitude of the sensor response for both BTO and PZT filled compositions were similar throughout the thermal loading range, with PZT filled compositions having a higher capacitance overall.

The thermal effects on the sensor response were two-fold, including thermal drift and reduction in the sensor response. Thermal drift, defined herein as the undesired change in capacitance as a function of temperature, is nearly linear for the BTO filled composition down to $0{ }^{\circ} \mathrm{C}$, dropping more quickly at lower temperatures. The PZT filled composites experienced a more linear thermal drift on average, though the difference was not significant. Both compositions experienced decreased response to mechanical loading correlating with reduced temperatures. This effect was most significant at $-80{ }^{\circ} \mathrm{C}$, where the response dropped by more than half. Thermal drift, and the reduction in the sensor response, are believed to be most significantly introduced by the polymer, theorized from these results to approach a glass transition temperature just below $80{ }^{\circ} \mathrm{C}$. This phase transition causes stiffening of the matrix, 
which increases the elastic modulus (reducing strain) and subsequently increases internal stress which is known to decrease permittivity [186]. Increased nonlinearity in the thermal drift of BTO was expected, as the Curie temperature of the $100 \mathrm{~nm}$ BTO is near $120^{\circ} \mathrm{C}$ and other phase transitions are found below 120 ${ }^{\circ} \mathrm{C}$.

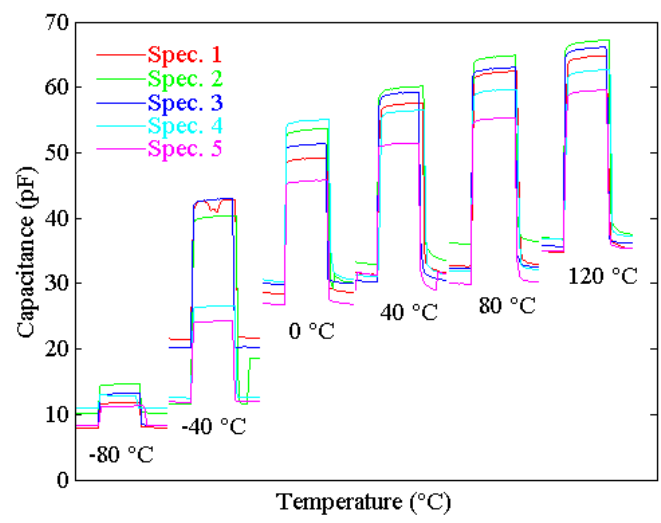

Figure 51. The sensor response (capacitance) to a mechanical step load from $0 \mathrm{kPa} \rightarrow 360 \mathrm{kPa}$ as a function of temperature for BTO filled composite specimens.

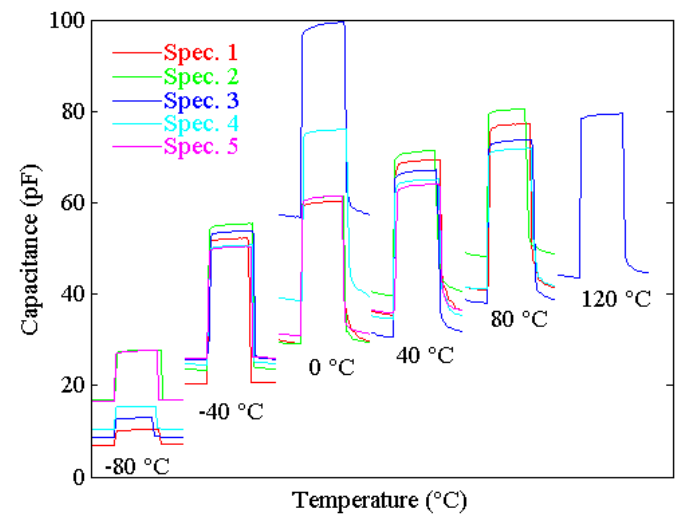

Figure 52. The sensor response (capacitance) to a mechanical step load from $0 \mathrm{kPa} \rightarrow 360 \mathrm{kPa}$ as a function of temperature for PZT filled composition specimens.

The PZT filled composite specimens 3 and 4 displayed a rise in capacitance at $0{ }^{\circ} \mathrm{C}$, and specimens 2 and 5 showed a similar rise at $-80^{\circ} \mathrm{C}$. The cause of these increased capacitance levels is believed to be caused by leakage currents which did not percolate through the entirety of the specimen, resulting in a reduced equivalent thickness and increasing the capacitance. As shown in figure 53, the capacitance of some specimens jumps between the expected magnitude, and the elevated magnitude under discussion. It is believed that PZT particles may have temporarily aligned in the microstructure, causing 
the theorized reduction in the equivalent thickness caused leakage currents. While specific deformation characteristic of cross-linked polymers is under debate, it has been reported that nonaffine strain (not uniform along the axis of stress application) occurs on a microstructural level for highly flexible polymers up to the point of rigidity percolation of the network (when pores collapse to the point that non-porous mechanical properties are restored to the bulk material despite the persistence of a porous phase) [187]. Additionally, it has been shown that mechanical properties of the cross-linked material decay with increased volume loading of the filler. This finding is contrary to the expected results, and is believed to be caused by reduced cross-linking with increased filler concentrations [188]. However, this theory lacks concrete evidence due to a lack of detailed knowledge of the microstructure, and requires additional knowledge of the nano-scale mechanical properties to be expounded.

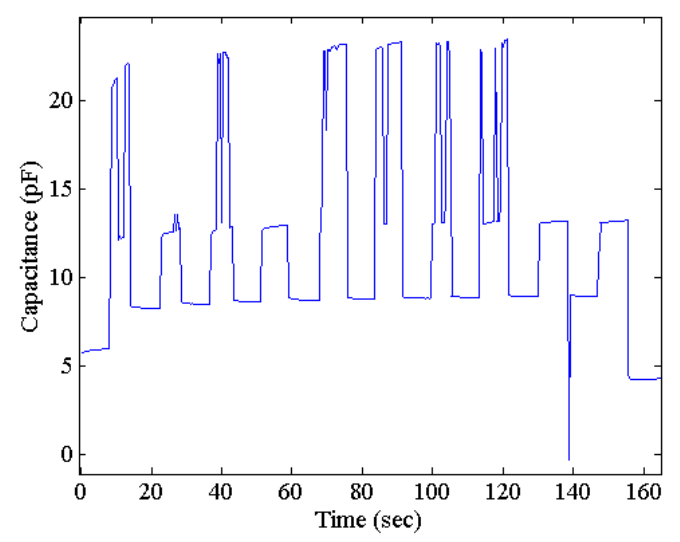

Figure 53. Sensor response (capacitance) of PZT filled composite specimen 3 at $-80{ }^{\circ} \mathrm{C}$, exhibiting sporadic behavior over the course of 10 step loads from $20 \mathrm{kPa} \rightarrow 360 \mathrm{kPa}$. This behavior was caused by partial leakage currents between PZT particles within the bulk

At $120^{\circ} \mathrm{C}$, the PZT filled sensor composition exhibited a normal response under $20 \mathrm{kPa}$, but was found to short circuit at $360 \mathrm{kPa}$. Specimen 3 of the PZT filled sensor composition was the only one which did not short, as shown in figure 53. The other specimens did not experience visible degradation, and the response immediately returned to normal when the pressure was reduced to $20 \mathrm{kPa}$. An industrially manufactured PZT (Piezo Kinetics Inc., PKI-502) with a particle size of $1 \mu \mathrm{m}$ was substituted and tested for comparison, yielding identical results. The sensor thickness was increased from $9 \mu \mathrm{m}$ to 18 $\mu \mathrm{m}$, which delayed the time to shorting, but did not enhance the long term properties of the senor under repeated loading of $120^{\circ} \mathrm{C}$ and $360 \mathrm{kPa}$. At $80^{\circ} \mathrm{C}$, specimen 5 was the only one to short circuit under pressure. The remaining specimens performed well at $80{ }^{\circ} \mathrm{C}$, not experiencing shorting. In contrast, no specimens with the BTO filled composition experienced shorting. 
Purely ceramic PZT films of a similar thickness $(\sim 10 \mu \mathrm{m})$ used for micro-electromechanical systems have been found to short circuit unexpectedly and without explanation in literature [189] [190]. Further review of advanced dielectric properties in random access memory applications provides a thorough and detailed explanation of variables affecting the breakdown voltage in dielectric materials. A summary by J.F. Scott shows both analytically and experimentally that increased temperature linearly decreases the breakdown voltage of a capacitor [191]. This explains the cause of the short circuit found to occur only at elevated temperatures.

While the reduced film thickness linearly increases capacitance, it is found to exponentially increase leakage currents which cause the breakdown current. The breakdown time was also shown to rise exponentially with reduced voltage. The combination of these effects explains the delayed time required for shorting of the thicker sensors. While Scott did not show experimental results for BTO, he showed that the breakdown current for PZT is significantly lower than that of barium strontium titanate (BST) given identical processing and environmental conditions. This further strengthens that theory that PZT was the cause of the short circuit experienced.

J.F. Scott also showed that the breakdown current was linearly dependent on the electrode work function, with BST between aluminum electrodes (which were used in this work) having a breakdown voltage four times lower than the same BST film between platinum electrodes. Additional specimens were prepared, sputtering $500 \mathrm{~nm}$ of platinum onto Kapton® film and casting the sensor composition as previously described. The PZT filled composite specimens were prepared on platinum electrodes as shown in figure 54. Since the 'sandwich' style electrical contacts could not be used (Kapton ${ }^{\circledR}$ film is not conductive) the back contact was prepared by placing copper tape on the platinum lead, and soldering the silver micro-wire directly to the lead (figure 54). There were no notable improvements found in specimens prepared on platinum electrodes, though future work should include substitution of the active arm electrode from copper foil to platinum foil. 

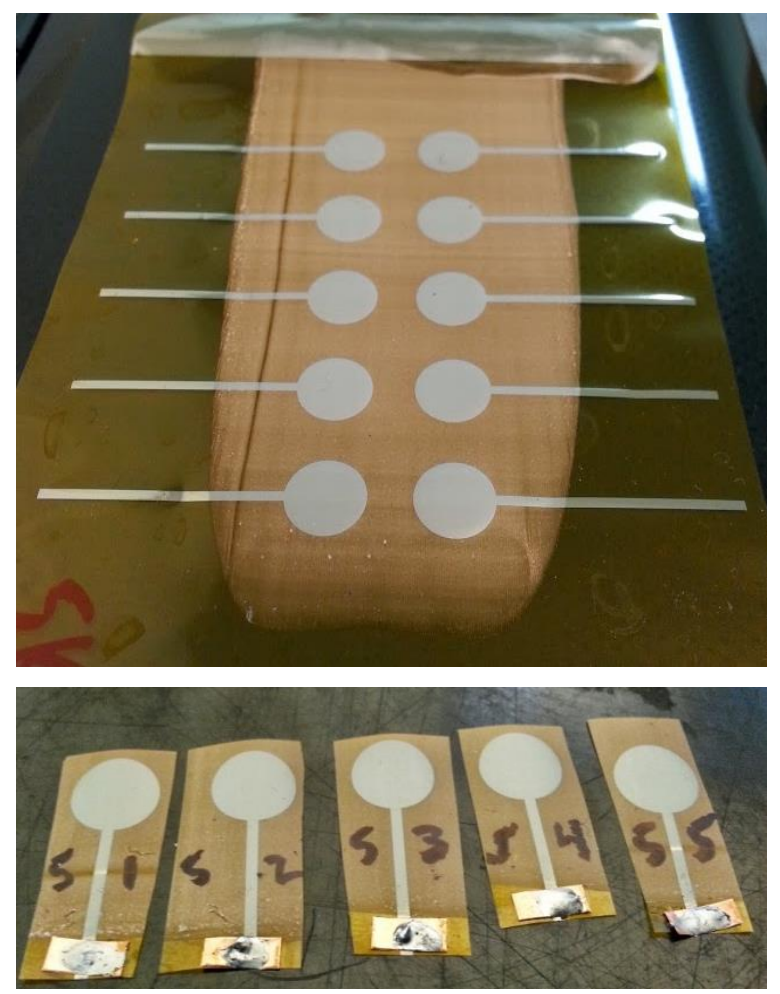

Figure 54. A piece of Kapton ${ }^{\circ}$ film was used as a substrate, with platinum electrodes deposited by DC sputtering, and PZT filled sensor composition cast using a mayer rod.

Room temperature testing was performed to evaluate the character of the two sensor compositions (quasi-static relationship between stress and capacitance). Using the final loading ramp from the 10 cycle loading sequence applied to each specimen, a summary of results was compiled in a capacitance versus stress plot for BTO and PZT filled compositions in figures 55a and 55b, respectively. As with thermomechanical testing, both the BTO and PZT filled compositions gave similar trends, with PZT filled compositions having a slightly higher capacitance. The non-linear, strain hardening trends were expected on account of the porous phase. The sensor character increases in linearity with applied stress as strain causes pores to collapse, and the flexible polymer network approaches the point of rigidity percolation [187]. Both compositions displayed an excellent response to mechanical loading, nearly doubling in capacitance under the applied stress. The sensors also displayed excellent resistance to noise, as the raw, unfiltered data plotted in this work shows a signal-to-noise ratio of over 500 on average.

The effect of ceramic fillers and porosity on the sensor response were demonstrated by characterizing a single phase polymer film. Using Arathane 5750 free of solvent or any other additives, a thin-film was cast using identical methods as the composite sensor fabrication. These unmodified sensors were characterized at room temperature, showing the capacitive response to a quasi-static load application (Figure 55c). The initial, unloaded capacitance was slightly lower than the three phase sensors. More 
importantly, the unmodified polymer capacitive response under mechanical loading was $\sim 7$ times lower than the modified sensor compositions. The non-porous films were expected to display a linear capacitive response to the load application. However, casting the solvent free material resulted in a viscous ink which maintained the ridges inherently formed during casting with a mayar rod. These ridges were equivalent to porosity, resulting in non-linear trends. While the specimens character differed because of inhomogeneous ridge patterns, the overall trend showed increased linearity as compared with the porous counterparts.

An observed degradation of the sensor microstructure causing notable changes in the sensor response across sequential runs in a loading schedule, referred to as sensor drift, was observed upon initial loading of sensor specimens. Shown in figure 56, this drift is demonstrated by showing a change in the capacitive response of a single sensor specimen during the first ten loading schedules after fabrication. Large changes in the capacitive response during initial loading runs for the composite, three phase sensors (figure 56a and figure 56b), indicate notable changes to the sensor microstructure.

The capacitive response of the composites sensors maintain the same basic trendline, changing primarily in overall magnitude (the capacitance is slightly increased with sequential loading runs). Since composition of the composite does not change between loading runs, the permittivity of the material should be constant. Therefore, it is reasonable to assume that collapsing of the pores is directly responsible for the increased capacitance between runs (sensor drift) through the mechanism of decreased film thickness. This drift is not a concern however, as drift is shown to be negligible after only a few loading cycles. The unmodified Arathane 5750 thin-film shows almost no changes in the capacitive response between loading runs (figure 56c), further indicating that the complex three phase system is responsible for the observed drift.

After performing 100,000 loading cycles from $16 \mathrm{kPa}$ to $280 \mathrm{kPa}$, the sensors were again characterized using a loading rate of $120 \mathrm{kPa} / \mathrm{min}$. The trend for each specimen is plotted before and after cyclic loading (figures 57 and 58), showing the effect of the loading schedule on the magnitude and trend of the sensor response. The capacitance of the preloaded specimens was expected to increase after the application of cyclic loading, as collapsing pores and degradation of the microstructure reduce the thickness of the film. However, comparison shows that the sensor response under preload was not significantly affected by cyclic loading. The sensor response was also expected to decrease as reduced porosity, plastic deformation on pore boundaries, and settling of the microstructure increased the elastic modulus. Both BTO and PZT filled compositions experienced similar degradation, with the response diverging by an average reduction of $5 \mathrm{pF}$ with application of a $360 \mathrm{kPa}$ load. 

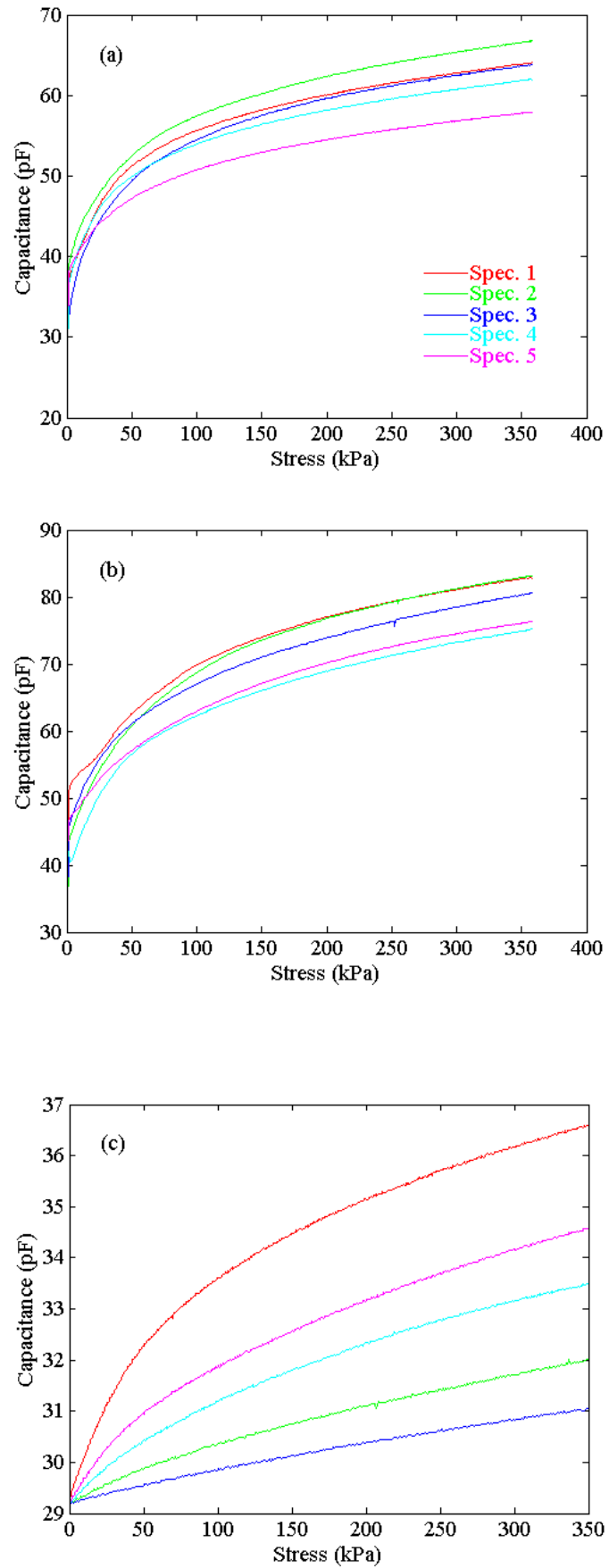

Figure 55. Capacitive response of five specimens for each sensor composition under quasi-static load application at room temperature: a) BTO filled compositions, b) PZT filled compositions, and c) unmodified Arathane 5750. 

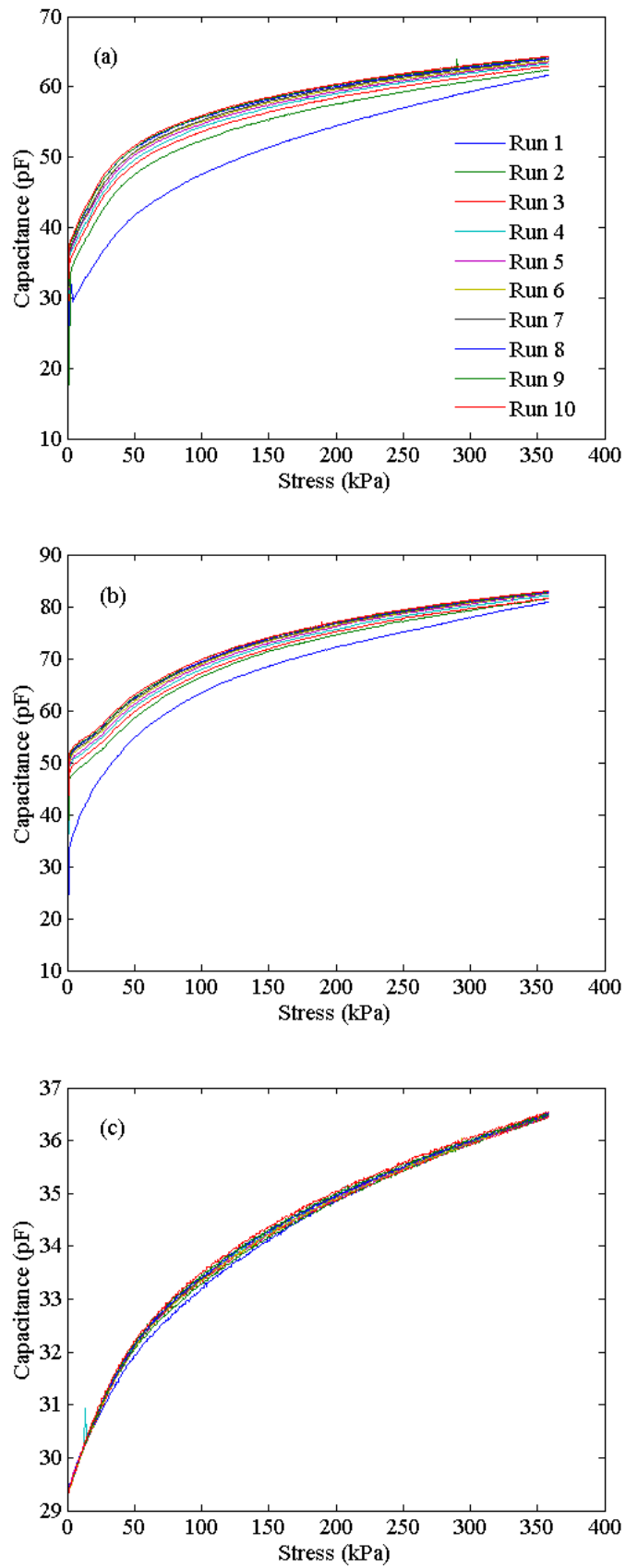

Figure 56. Ten loading runs performed on specimen 1 for each sensor composition showing the capacitive response under quasistatic load application at room temperature: a) BTO filled compositions, b) PZT filled compositions, and c) unmodified Arathane 5750. 


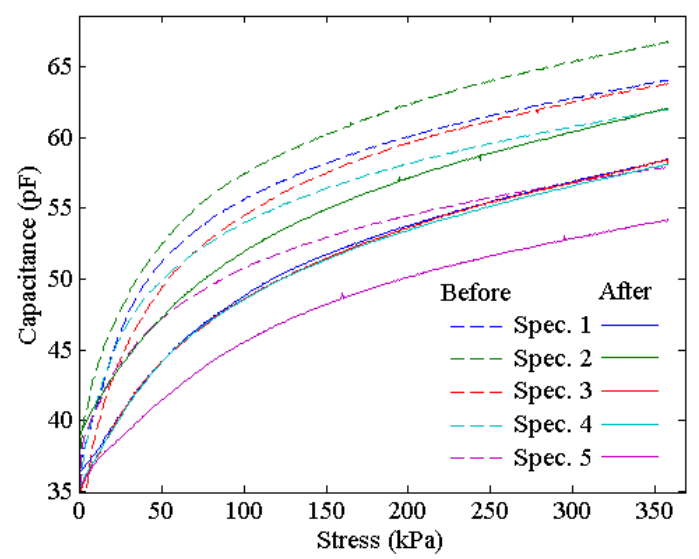

Figure 57. Sensor response (capacitive) of BTO filled sensor composition before and after undergoing 100,000 step loading cycles from $20 \mathrm{kPa} \rightarrow 360 \mathrm{kPa}$.

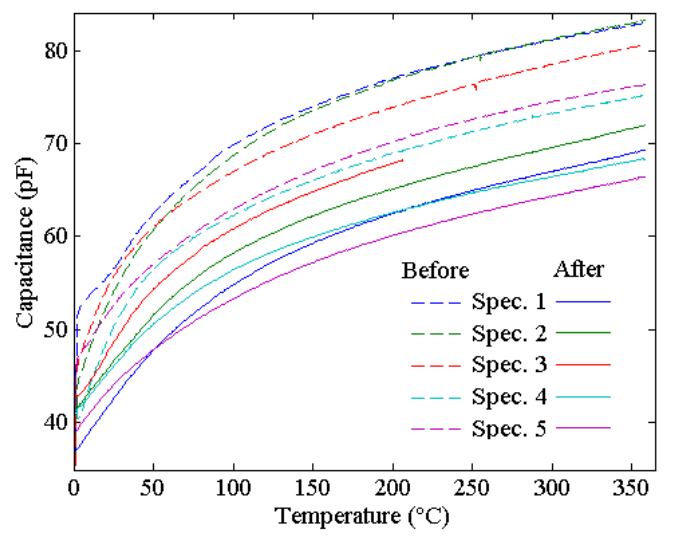

Figure 58. Sensor response (capacitive) of PZT filled sensor composition before and after undergoing 100,000 step loading cycles from $20 \mathrm{kPa} \rightarrow 360 \mathrm{kPa}$.

Using a load of $360 \mathrm{kPa}$, specimens were loaded for $24 \mathrm{hrs}$ to evaluate sensor drift of static loads in atmospheric conditions. During the course of the long term load, specimens experienced some isolated points of noise. The data was processed with simple filter and smoothing techniques, which implemented a 3 point floating average and removed single outlying points further than $1 \mathrm{pF}$ from the neighboring average. An average of 5 outlying points per hour was removed from the data, resulting in the results shown in figure 59. 

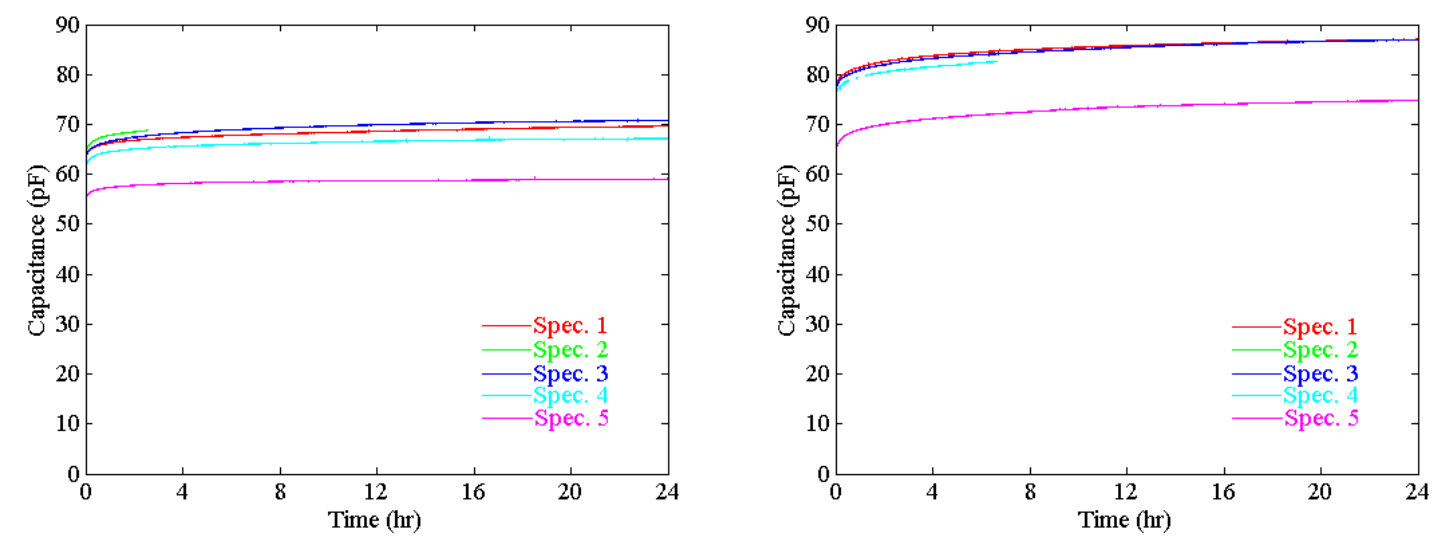

Figure 59. Sensor response over time for BTO filled (left) and PZT filled (right) compositions, with an applied static load of 360 $k P a$.

Results show that the PZT filled composition to have a greater capacitance than the BTO filled composition, as was observed with previous loading schedules. Error in the sensor response was found to be similar for both compositions when compared with the initial response, experiencing combined hysteresis and drift of $10 \%$ of the initial sensor response on average. The character of these errors is observed in more detail in figure 60, where the capacitance of each specimen is normalized by its maximum. It is believed that the initial change in capacitance during the first few minutes is dominated by viscoelastic properties of the polymer material, known as a rate dependent hysteresis. This hysteresis is removed after unloading of the specimen, where drift is caused by damage of the microstructure which permanently alters the response. Work in literature has shown that the elastic modulus, internal stress, and strain vary along the transverse axis of the polymer film when it is rigidly bound to a metal substrate [61]. It is believed that these attributes contributed the viscoelastic effects observed here. These effects could be reduced in future work by implementing a substrate similar to that shown in figure 54 .

During the next $4 \mathrm{~h}$ of testing, the magnitude of the subsequent error exponentially decays, as the viscoelastic creep effects are reduced and the drift effects begin to be introduced. Drift is caused by the shifting and settling of the nano-particles, and creep in the polymer microstructure resulting in plastic deformation around pore boundaries. The composite films containing BTO filler experienced greater error during the first $4 \mathrm{~h}$, indicating increased viscoelastic effects. Preparation of the filler's surface chemistry in nano-composites is known to affect the mechanical properties more significantly than other factors [87], and is likely the cause of this error. As discussed in section 5.2.1, the surface chemistry of PZT particles were carefully prepared and BTO particles were used as received from industry.

The breakdown voltage of the films continued to effects the results, as some specimens continued to short during loading. Seen in figure 59, PZT filled composite specimen 4 and BTO filled composite specimen 2 experienced shorting after a small amount of time, and PZT filled composite specimen 2 
failed entirely. For this reason, it is believed that leakage currents also contributed to progressive error in the sensor by reducing the equivalent thickness of the films as creep and settling/shifting of the filler promoted localized leakage by reducing inter-particle spacing.

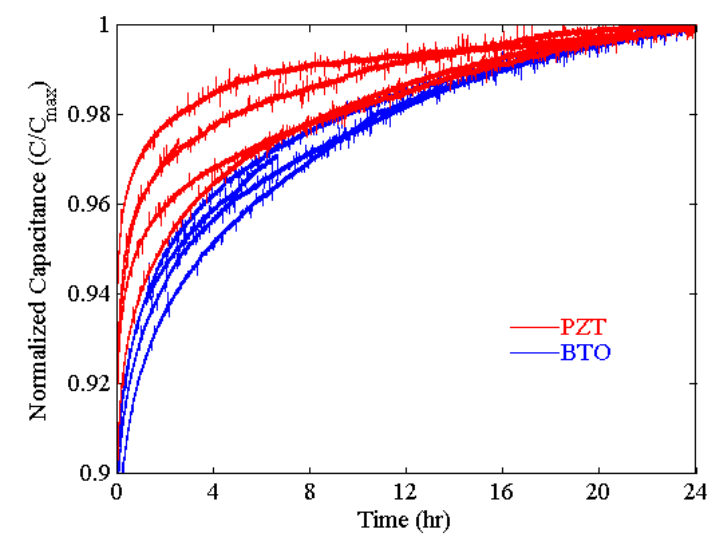

Figure 60. Normalized sensor response over time, with an applied static load of $360 \mathrm{kPa}$.

After completing all loading cycles, the cross-section of the film was observed using a SEM. The micrograph in figure 61 shows no notable changes to the thickness or microstructure of the film, nor any microscopic change in the adhesion between the film and the aluminum substrate.

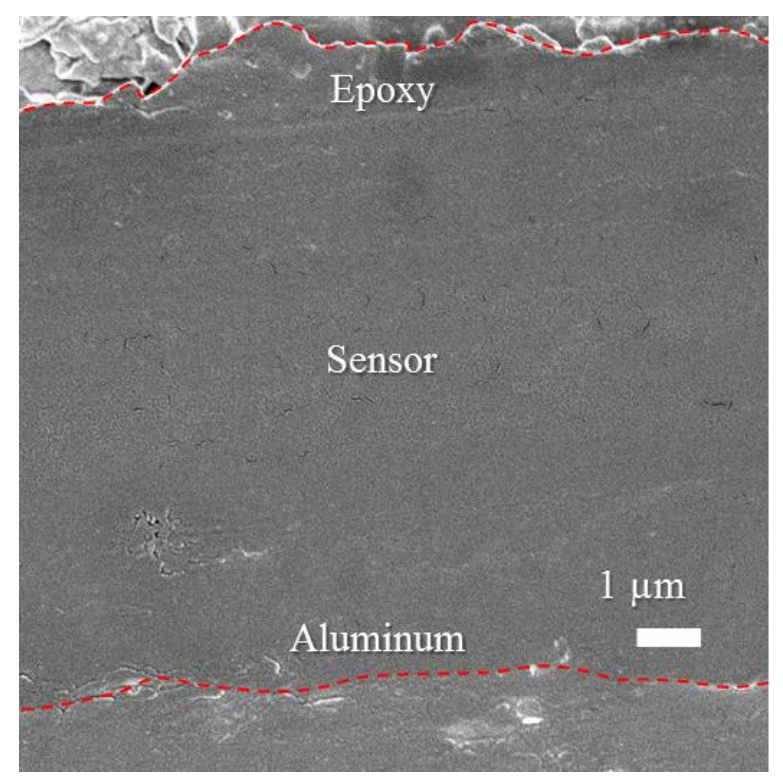

Figure 61. SEM micrograph showing cross-section of PZT filled composite specimen 1. 
Testing for outgassing did not show any mass loss, though two sets of specimens showed a small mass gained (table 1). The cause of this error is not believed to be contamination, but rather inconstancy with the weighing scale at small magnitudes. Assuming the weight of the sensor composition is the difference between bare aluminum substrates and the sensor specimens, approximately $17.5 \mathrm{mg}$ of material was evaluated for each composition. If the error found between the reference material (aluminum substrate) and sensor composition is assumed to be the maximum weight loss which could have occurred $(0.1 \mathrm{mg})$, the maximum total mass loss (TML) would have been $0.57 \%$. The maximum TML for meeting the referenced standard is $1 \%$, which means the composites passed by a factor of almost 2.

Table 6. Outgassing data for BTO and PZT filled compositions.

\begin{tabular}{c|ccc}
\hline Weight & BTO Filled Composition & PZT Filled Composition & Aluminum \\
\hline Before Vacuum $(\mathrm{mg})$ & 148.5 & 148.6 & 131.5 \\
After Vacuum $(\mathrm{mg})$ & 148.5 & 148.8 & 131.6
\end{tabular}

The final sensor composition was implemented into a preliminary sensor packaging design, demonstrating preparation of a flexible sensor array. The sensor packaged consisted of 5 layers (figure 62), making use of the proposed platinum electrodes and elastic Kapton® film substrate. A four sensor array was used to demonstrate the ability to recognize spatial distribution of forces. Increased spatial resolution can be attained by simply modifying the electrode pattern on which the sensor film is cast. Embedding of the sensor package was proposed for protection from environmental effects such as electron bombardment, radiation, atomic oxygen, and damage caused by point concentrations during metal-on-metal contact. Shown in figure 63, a minimally intrusive design was proposed for a two sensor array using stainless steel foil to shield the sensor array. 


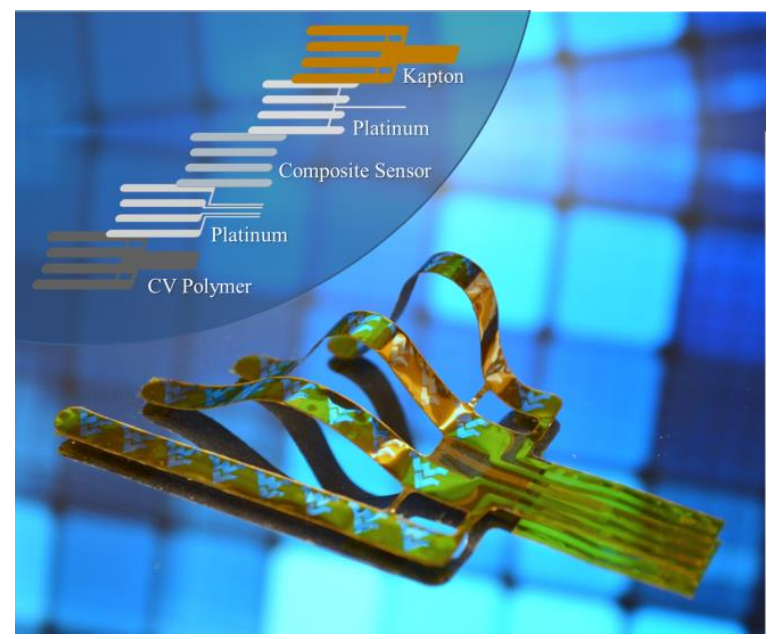

Figure 62. Proposed sensor package for applied use of sensor composition.

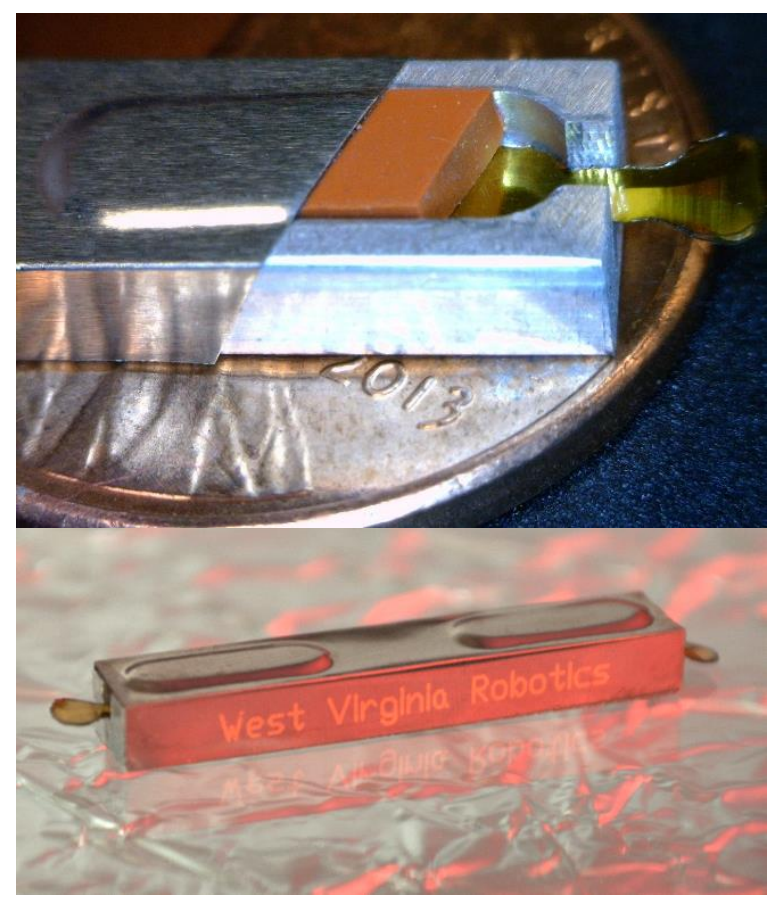

Figure 63. Proposed technique for minimally intrusive embedding in existing end effectors.

\subsection{Conclusions}

The design of a high dielectric, low elastic modulus composition was proposed using a 0:3:3 connectivity ceramic/polymer/porous composite. Variables such as composition, volume content, and size distribution of constituents were carefully optimized. Considerations for variables included effects on mechanical, thermal, and electrical properties on both a micro and macro level. Realization of the proposed sensor 
composition was achieved by using combination of industrially available materials and in-house synthesis of nano-particle PZT. Selection of appropriate processing methods, including substrate and electrode properties, were defined as important parameters in the design. Two final sensor compositions were fabricated, differing by the composition of the filler material. The preferred filler composition was PZT, which is not prone to thermal drift in the desired temperature range but experiences a low breakdown voltage. BTO was used for its increased breakdown strength, despite having a Curie temperature near the desired working range.

Following fabrication of the proposed sensor, characterization was performed. Testing included thermomechanical loading, room temperature characterization of the sensor response trend, cyclic loading, long term loading, and outgass testing. The results showed that the PZT filled composition reached the breakdown voltage with combined thermomechanical loading of $120^{\circ} \mathrm{C}$ and $360 \mathrm{kPa}$, causing the sensor to short. However, reduction of the either the thermal or mechanical load restored functionality to the sensor without permanent damage. Otherwise, both compositions performed well under the simulated space conditions. As compared with the BTO filled composition, PZT filled composite specimens exhibited a higher response, reduced thermal drift, and increased linearity in creep effects during long term loading. Applied use of the sensor composition was carried out by implementing flexible electrodes, and flexible electrically insulating packaging. Protection from additional environmental effects was proposed by embedding the sensor package into the metal end effector, using a minimally invasive approach. 


\section{Chapter 6. Embedded sensor design and future work.}

\subsection{Introduction}

The NASA Goddard Space Flight Center (GSFC) Robotic Refueling Mission (RRM) is preparing for docking of robotic systems on satellites in earth orbit, performed by grappling of the marman ring which is mounted on all satellites for deployment into space. This process is being demonstrated in a lab environment, using a mock satellite configured on an automated robotic space simulator as shown in figure 64. Force and spatial awareness is necessary to properly grasp the marman ring, providing knowledge of contact force, timing, and spatial distribution of forces.

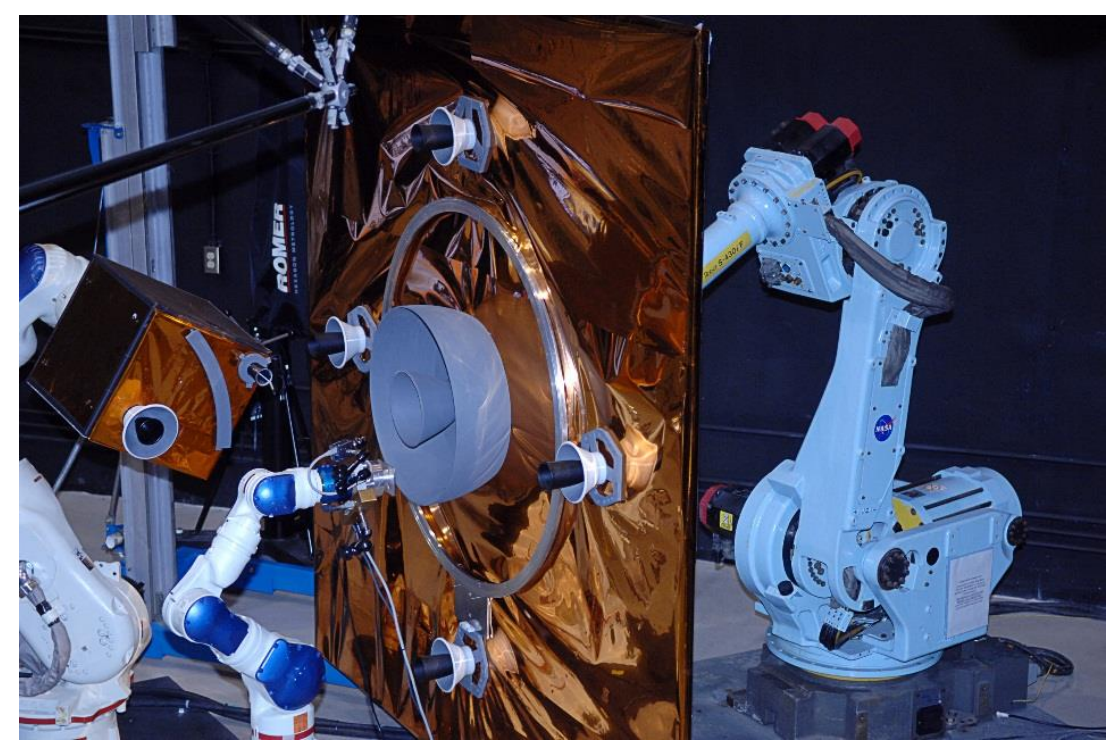

Figure 64. A marman ring mounted on a mock satellite, with an automated robotic space simulator for lab demonstrations of proposed in-orbit tasks.

The marman ring gripper tool (figure 65) was designed specifically for initial grappling of satellites in earth orbit, allowing for secure docking of the robotic equipment for refueling and repairs. The area highlighted in orange signifies the locations where force and spatial awareness information are necessary. The contact surfaces in this task will endure high forces, metal-on-metal contact, and strict geometry requirements. 


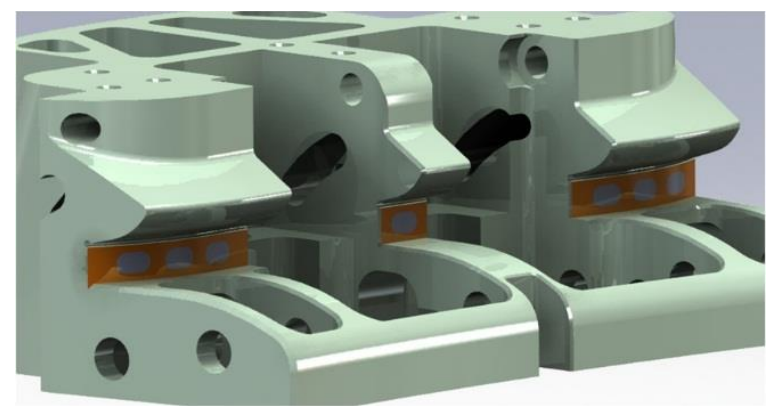

Figure 65. NASA GSFC RRM Gripper tool for docking robotic equipment on the satellite marman ring for refueling and repair of satellites in earth orbit.

The simple implementation of a thick-film sensor packages does not present a large enough compliant layer to distribute point forces between the metal gripper tool and metal marman ring. Machined metal surfaces can surface defects in the range of $25-150 \mu \mathrm{m}$, creating point and line concentrations in the sensor film. The resulting force concentrations are difficult to quantify, and could damage the sensor package over time. For this reason, an additional compliant layer is necessary for implementing the capacitive sensor film in the marman ring gripper tool. However, strict requirements prevent the implementation of additional compliant material. Such a layer would distort the carefully designed gripping geometry, preventing proper docking, as well as introducing chattering into the automated grappling algorithm.

\subsection{Experimental}

The NASA RRM marman ring gripper tool was used as a potential force sensor array recipient for demonstrating the sensor embedding process. In order to make use of existing gripper tools, rather than designing and manufacturing new gripper tools, a small cavity was milled into the contact area of the gripper to provide a small depth for embedding sensor materials. This also allowed for an additional compliant layer, used to distribute point concentrations from contact with micro-roughness on metal parts. The actual gripper tool was not available for modifications and lab testing, so a model was fabricated. The dimensions shown in figure 66, this prototype was fabricated from aluminum with a small cavity machined for embedding of the sensor packages (shown in red). The machining of cavities for the prototype was performed using $1 / 8$ in and 1/16 in end mill bits, allowing for large radius edges to simplify the machining process. The completed prototype, with milled cavities for embedding, is shown in figure 67. 


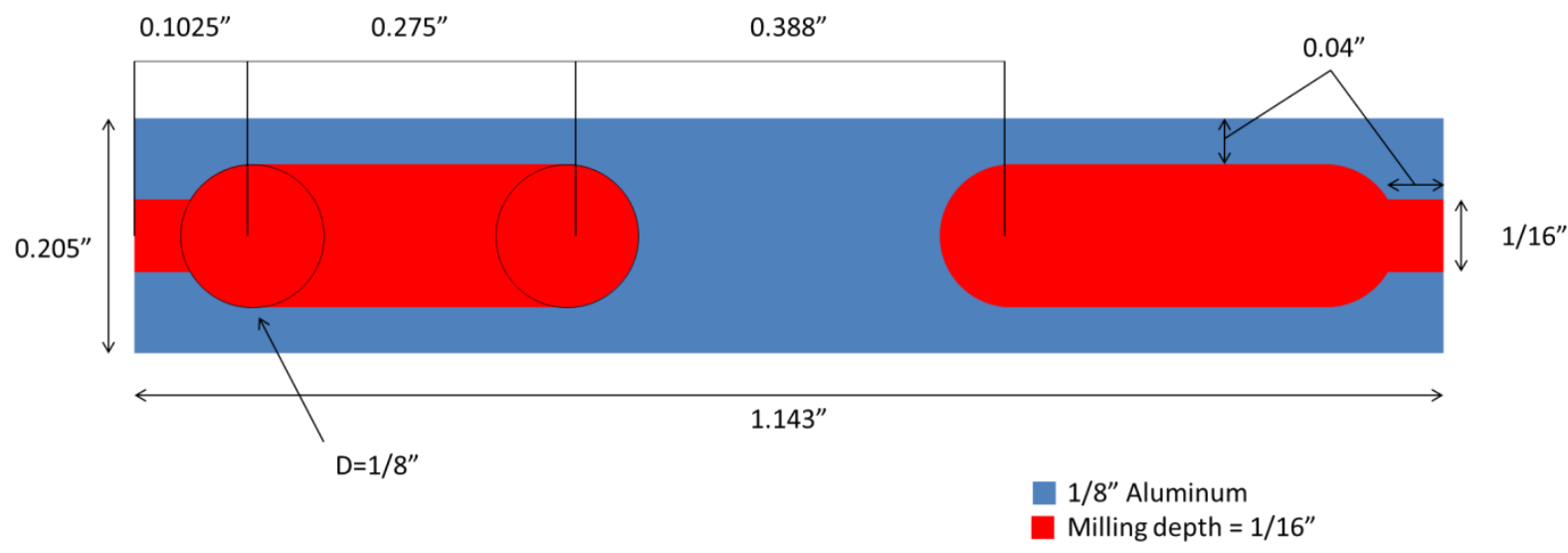

Figure 66. Gripper tool prototype, with dimensions matching the docking area shown in orange in figure 65. In this figure the blue represents the aluminum bulk material, and the red represents cavities machined to a depth of 0.125 " providing area for embedded sensors.

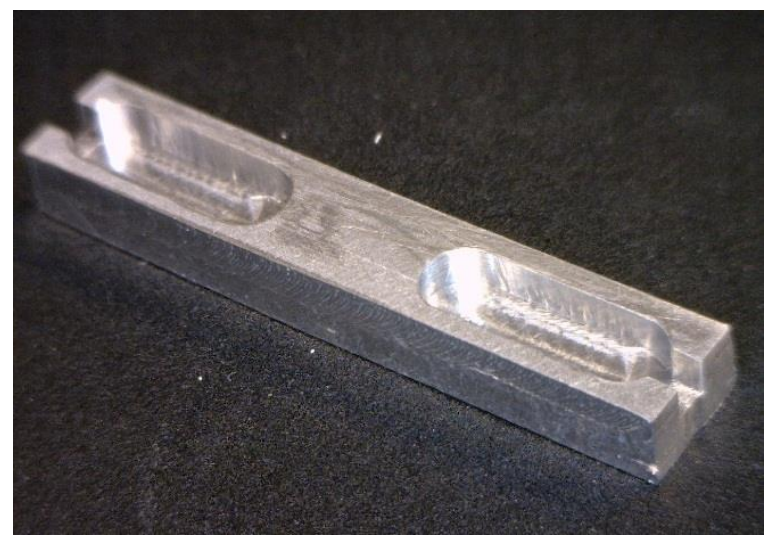

Figure 67. Gripper tool prototype machined from aluminum, with end milled cavities for embedding sensors.

A new sensor package was fabricated to meet the specific needs and dimensions for the embedded prototype. Characterization of the sensor composition in chapter 3 was performed without a substrate, and in chapter 4 with a metal foil substrate for ease of electrical acquisition. The sensor packages demonstrated in chapter 1 were further developed in this chapter, with basic modifications to further improve the sensor response. The primary difference is that the electrodes were sputtered onto the substrate films rather than onto the porous sensor composition. This prevented deposition of electrodes deep into the pores of the sensor composition, reducing the effective thickness of the sensor film.

The fabrication of the sensor package was initiated by depositing platinum electrodes onto Kapton® film using photolithography processes. The film was placed onto a polished aluminum wafer and covered in AZ 5214 E image reversal photoresist. The vacuum chuck on the spin coater was then ramped at 330 $\mathrm{rpm} / \mathrm{sec}$ to $440 \mathrm{rpm}$, where the $440 \mathrm{rpm}$ was maintained for 10 seconds before ramping at $440 \mathrm{rpm} / \mathrm{sec}$ to 
3,000 rpm. The 3,000 rpm was maintained for 15 seconds before the spinner was turned off. The film was removed and dried on a hot plate at $95{ }^{\circ} \mathrm{C}$ for three minutes. The patterned photomask was placed over the photoresist coated film, and aligned by hand on the film under a OAI UV flood exposure. Because of the large and simple nature of the pattern, the film was exposed for $10 \mathrm{sec}$ regardless of the lamp intensity, which can over the lifetime of the bulb. The film was then developed in AZ 310 MIF for approximately 30 seconds. DC sputtering was performed to deposit a platinum layer approximately 500 $\mathrm{nm}$ thick (estimated using a known deposition rate), and an acetone lift-off process was performed in a sonication bath to reveal the patterned electrodes.

Using the barium titanate sensor composition and mayer rod casting method (9.5 $\mu \mathrm{m}$ deposition thickness) described in chapter 4 , a thin-film sensor composition was cast over the sensor electrodes. Shown in figure 68 , the ends of the sensor electrodes were wiped clean with a clean room cloth wipe after casting the sensor composition, providing surface area to attached silver micro wires to the sensor electrodes. The Kapton® film substrates did not wet as well as the aluminum foil substrates during casting, and they experienced imperfection in the casting surface. This caused sensors to short circuit, as electrodes were not fully coated by the sensor material. For this reason, after the initial layer of cast sensor material was deposited and dried, a second layer was cast. The drying process was carried out precisely as described in chapter 4. Two identical films were prepared, and one film was cast with a third layer of sensor material before sandwiching the two films. The films were pressed tightly together and dried to create the final sensor package. After full sensor package was dried, a laser cutter was used to trim the individual sensors from the un-connected array. The flexible silver micro-wires were added using silver epoxy.

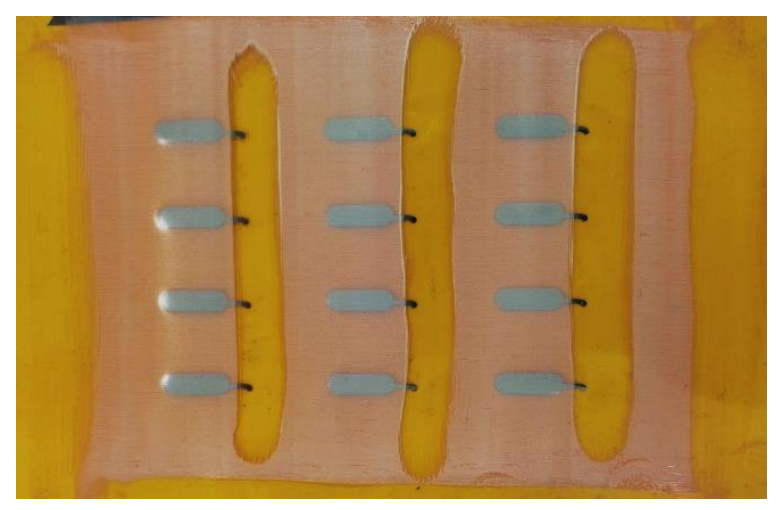

Figure 68. Kapton ${ }^{\circledR}$ film substrate, with platinum electrodes and BTO filled sensor composition cast using a mayer rod (9.5 $\mu$ m). The sensor composition was wiped from the electrode tips, providing access to mount silver micro-wires.

The completed sensor package was placed within the gripper tool cavity, surrounded on either side by a $127 \mu \mathrm{m}$ (5 mil) thick Mylar® layer to further increase the sensor life by removing frictional forces. The 
Mylar® film was also used to reduce the remaining cavity space. A 1/16 in silicone rubber was fit within the cavity, which protruded from the gripper surface by a small amount because of the underlying layers. The intended purpose of partially protruding the silicone layer was to allow for the layer to be collapsed below the surface of the gripper tool upon overloading, transferring forces to the surrounding metallic gripper and protecting the sensor from accidental overloading during grappling.

A metallic layer was used to contain and protect the embedded layers, shielding from electron bombardment, atomic oxygen, radiation, and harsh abrasion. In order to minimize the thickness of the metal foil, it was necessary to utilize a dense, stiff metal foil. Both aluminum and stainless steel foils were found to easily deform, though titanium displayed excellent strength properties as a protective sheath. The titanium foil was formed with hillocks before installation, using a hydraulic press. A Mylar® film was adhered to the foil using double sided tape. The film was then patterned using a laser cutter, and excess film was removed. The metal foil was placed into a hydraulic press with the patterned Mylar® film below, and a 1/8 in silicone rubber atop. The layers were then pressed to 5 metric tons, introducing the desired hillocks. The full embedded sensor prototype can be seen layer-by-layer with a cutaway view in figure 69. The full two sensor package in the gripper tool model is shown in figure 70.

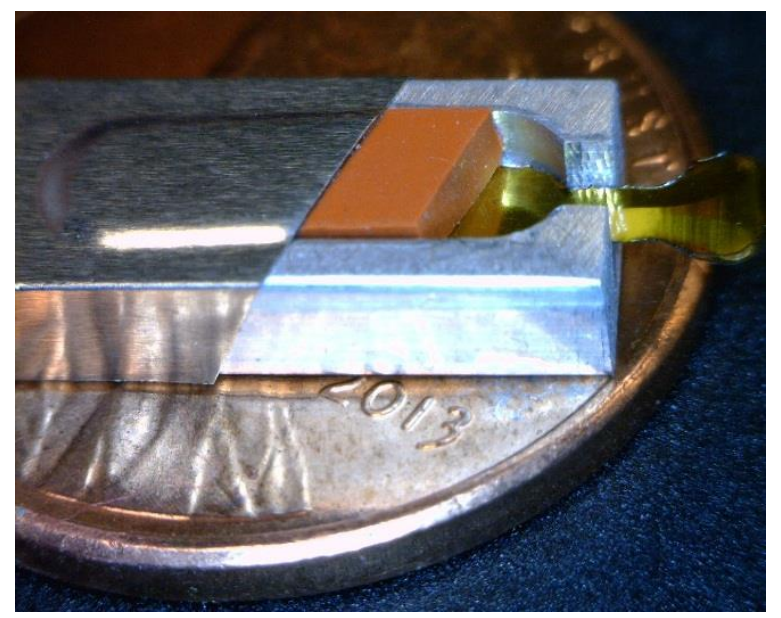

Figure 69. The embedded sensor prototype, shown with a cutaway view to reveal the sensor package, silicone compliant layer, and titanium foil cover. 


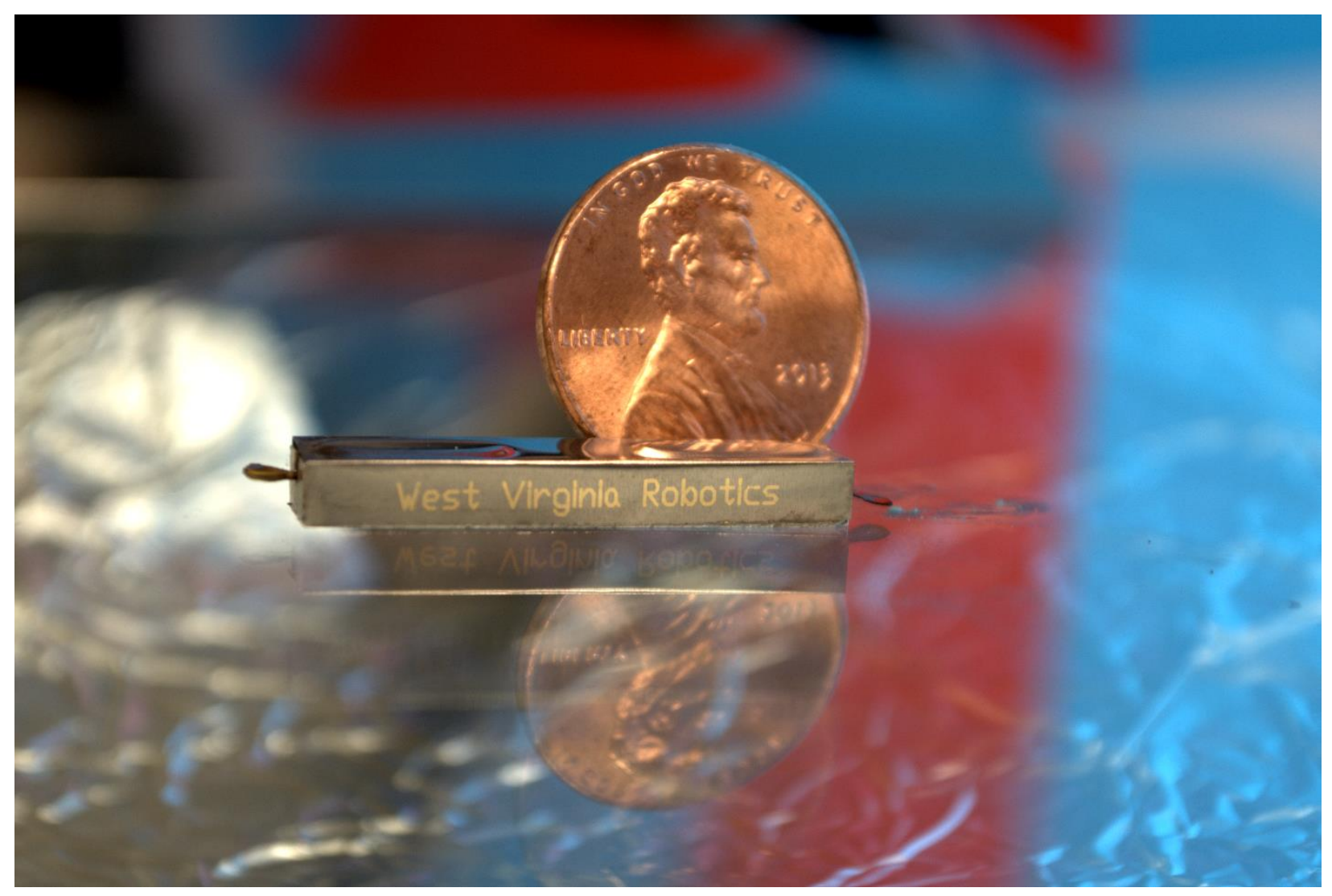

Figure 70. The fully complete marman ring gripper tool prototype, hosting two sensors.

Characterization of the embedded sensor array was performed by applying a mechanical load, and acquiring the electrical response (capacitance) of the sensor. In order to fully understand the effects of embedding, the sensor was characterized: 1) as a bare film, 2) within the gripping tool cavity with a silicone layer, 3) fully embedded with the metal foil as depicted in figure 71 . In this way, the effects of each constituent could be more fully explored. The mechanical load was applied with a Shimadzu AGS$\mathrm{X}$ loading stand.

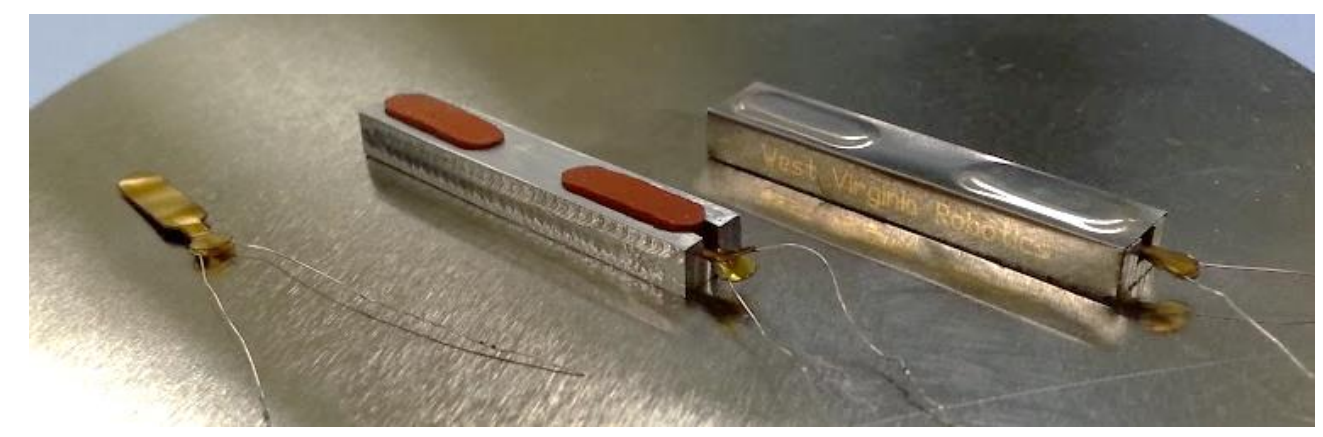

Figure 71. The three different testing configurations: 1) bare sensor package, 2) embedded sensor with rubber compliant layer, and 3) fully embedded sensor with metal foil cover. 
The upper (active) compression clamp was used as received from Shimadzu. In order to prevent accidental overloading from metal-on-metal compression forces after collapsing the small compliant layer in the embedded sensor, additional compliant layers were added in series. Shown in figure 72, the lower (static) clamp was prepared with compliant layers and a flat aluminum plate, providing both a buffer against accidental overloading during compression and metal-on-metal contact surrounding the embedded sensor prototype. Data acquisition with the LCR meter was performed at $1 \mathrm{~V}, 200 \mathrm{kHz}$, and was achieved by soldering the silver micro-wire directly to the LCR leads.

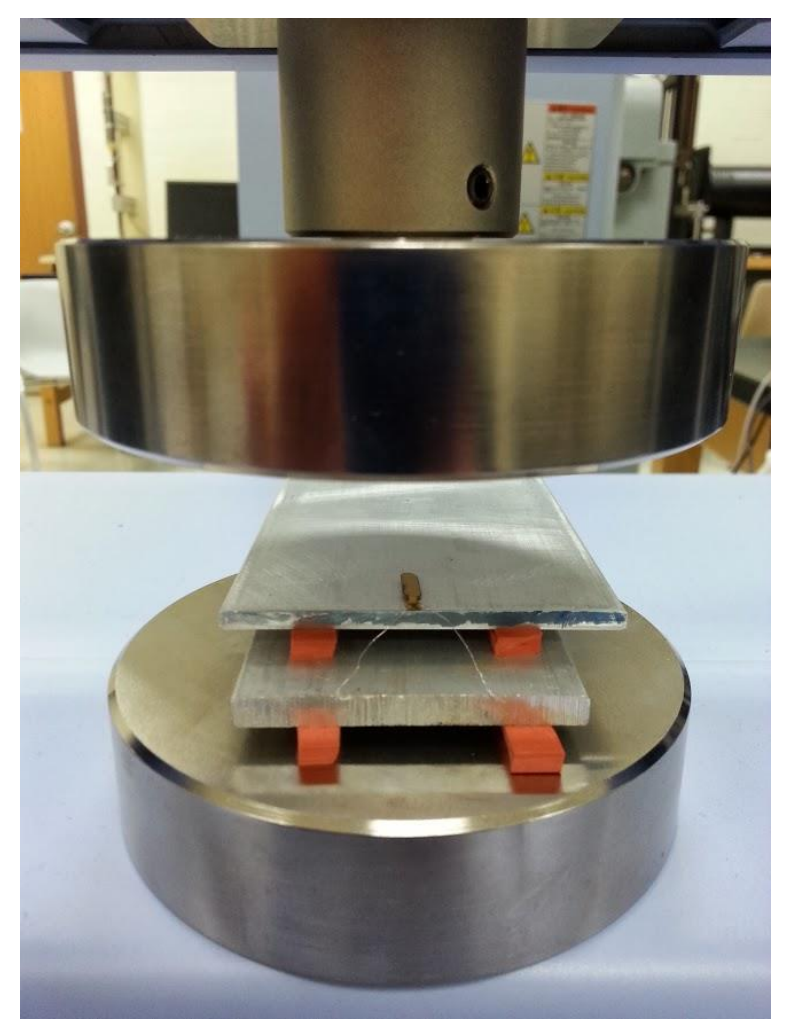

Figure 72. The Shimadzu testing setup with stock compressive clamps, the lower (static) clamp having been prepared with two compliant layers to prevent accidental overloading from metal-on-metal contact.

The mechanical loading was applied with a constant rate of displacement, rather than a constant load rate. This was done to promote safety and prevent accidental overloading of the electromechanical equipment, which could be caused by automated movement of the active clamp which accelerates the displacement speed to meet the requested loading rate. For the bare film sensor, a displacement rate of $0.25 \mathrm{~mm} / \mathrm{min}$ was used, and the test was completed with a load of $250 \mathrm{~N}$. A rate of $0.5 \mathrm{~mm} / \mathrm{min}$ was used to apply a final load of $250 \mathrm{~N}$ for both the embedded sensor with rubber, and the fully embedded sensor with added titanium foil. 
The Shimadzu data acquisition for force load was achieved at a rate of $10 \mathrm{~Hz}$, while the frequency for the LCR capacitance data varied, with an average acquisition rate of $\sim 3 \mathrm{~Hz}$. In order to plot the sensor response (capacitance) as a function of applied stress (force/area), two steps were taken. First, the force data was interpolated to provide approximate force data for each LCR time stamp. Second, it was assumed that all applied forces were perfectly distributed across the sensor face without any parallel contact of the active clamp on the gripper tool. This allows for a distinct surface area for load distribution in the stress calculations. The error in this assumption appears as an apparent loss in sensitivity, as additional force is applied and the capacitance does not change accordingly.

The order in which specimens were tested was alternated, preventing any potential progressive damaged caused during the loading schedule from being improperly correlated with a specific embedding constituent. Specimens A and $\mathrm{C}$ were first loaded in the gripper tool with both the rubber and titanium foil, where specimens B and D were first loaded in the gripper tool with only rubber. All specimens were tested as a bare film as the last step, since this process was expected to damage specimens. A total of three test runs were performed on each specimen, and four test specimens were tested. All test specimens were embedded within the same gripper tool prototype.

\subsection{Results and Discussion}

The combined capacitance and force data is displayed in figure 73 , showing the sensor response (capacitance) as a function of stress (force/area) for the bare film, the sensor embedded beneath rubber, and the sensor fully embedded with titanium foil. Each of the four specimens are plotted. The plot reveals the basic trends for each embedding constituent. When tested as a bare film (blue trendlines), specimens A, B, and C experienced a small change in capacitance. This indicates that the applied stress was not fully applied to the sensor face, but was partially maintained in parallel by contact of the two metal plates. In specimen D, the bare film experienced an excellent response, followed by sporadic behavior. It is believed that in this case the film was under a point concentration, causing excessive compression in an isolated location, which caused infrequent leakage currents.

After embedding the specimens within the gripper tool prototype and rubber compliant layer (green trendlines), results were improved. Specimens A, B, and C show an increased response, with less noise from leakage currents. Specimen D showed similar results as when it was tested bare (without embedding), and experience a short circuit with stress lower than where leakage currents were observed when tested as a bare film. This is a result of the rubber layer applying the full compressive load onto the sensor film, while the actual observed stress on the bare film is not accurately portrayed on the $\mathrm{x}$-axis since parallel loading occurring directly between the clamps bore some of the load. 
The addition of titanium foil over the rubber compliant layer more significantly contributed to dispersing point loads than the rubber compliant layer did on its own. A notable increase in sensitivity was observed in specimens A, B, and C, though specimen C short circuited at high loads. Additionally, the range at which the sensor could quantify the force magnitude was extended with metal foil for specimen D.
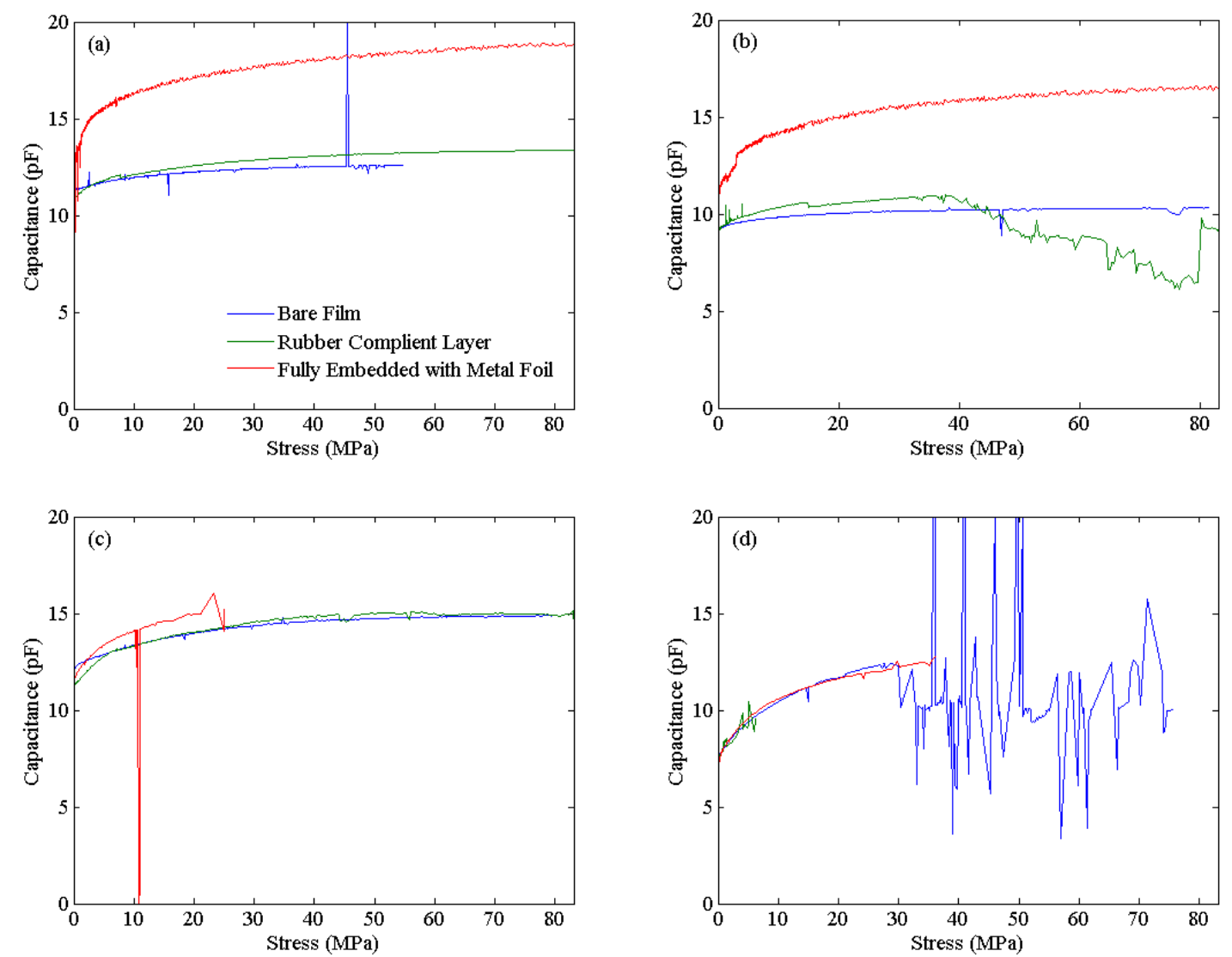

Figure 73. A total of four sensor specimens $(a-d)$ were tested in each of the three testing configurations. Each configuration is plotted for a single specimen, showing the difference in the sensor response (capacitance) under an applied stress.

The combined force and capacitance data for specimen A is displayed in figures 74-76, where the three consecutive loading schedules are displayed showing the sensor response (capacitance) as a function of stress (force/area) for the bare film, the sensor embedded beneath rubber, and the sensor fully embedded with titanium foil respectively. The bare film experienced noise and large spikes in capacitance during every run, demonstrating the anticipated leakage currents from point concentrations of the applied load. However, long term damage did not appear to be sustained, as successive runs did not display large amounts of drift in the response.

The embedded sensor with a silicone rubber compliant layer displayed excellent results, having negligible noise and drift. This shows that the compliant layer was successful in distributing point 
concentrations. However, as previously noted the sensor sensitivity (change in capacitance) was not significantly improved. It is theorized that point concentrations were only partially dispersed, resulting in largely anisotropic loading of the sensor face despite the improvement in stress distribution.

The additional of a titanium foil cover on the embedded sensor significantly increased the total change in capacitance over the full stress application. A small amount of noise was observed with the addition of the foil, and a large drift and same spikes in capacitance were observed in the first run. This drift was likely not caused by changes in the microstructure of the sensor composition, since such changes would be expected to occur more significantly from the bare sensor, and none were observed. This drift, which occurred during the first loading schedule, is believed to have been caused by the titanium film being contoured to the rubber compliant layer. After being intimately conformed to the rubber compliant layer, no additional changes were observed.

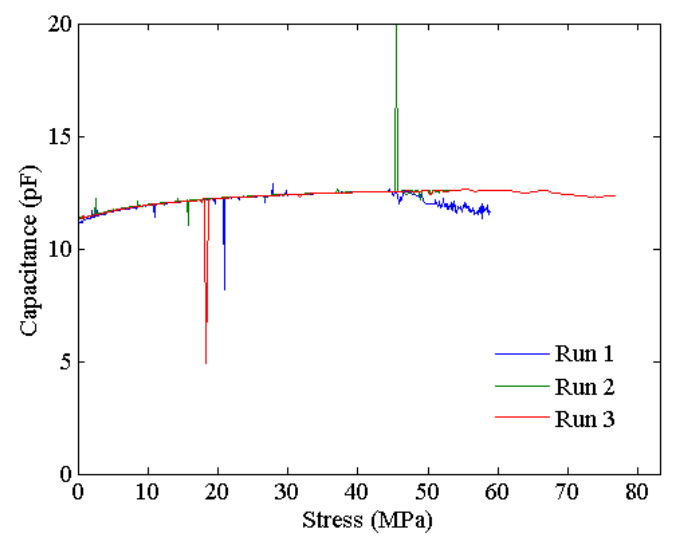

Figure 74. Sensor response (capacitance) as a function of applied stress (forcelarea) for specimen A, loaded as a bare film between two plates of metal.

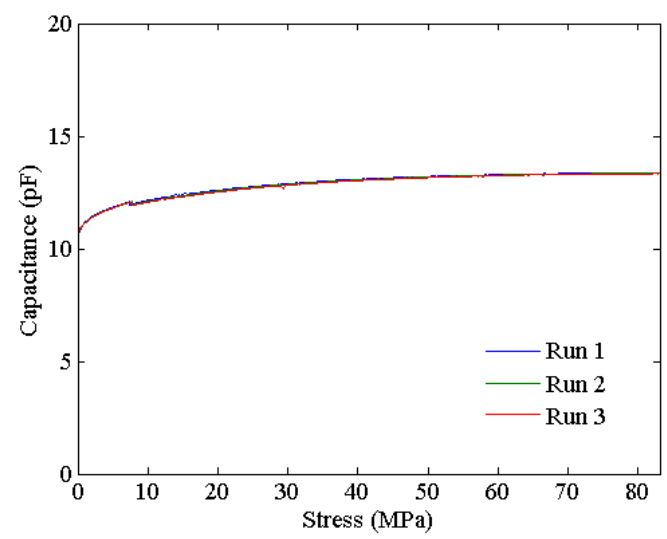

Figure 75. Sensor response (capacitance) as a function of applied stress (forcelarea) for specimen A, loaded as an embedded film within the gripper tool prototype with a rubber compliant layer. 


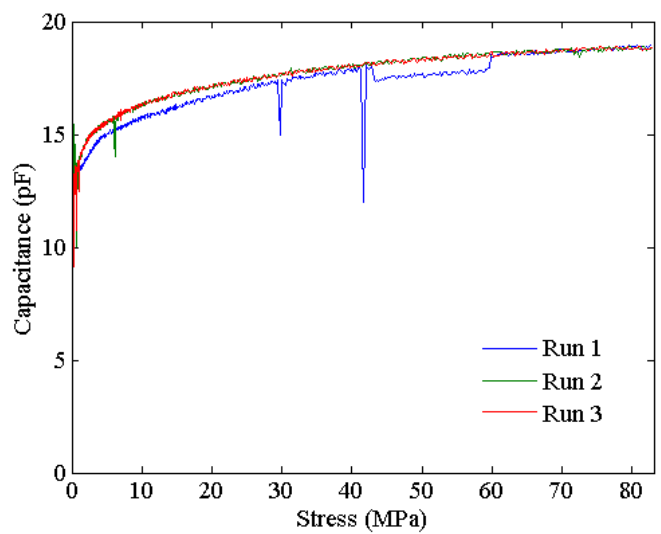

Figure 76. Sensor response (capacitance) as a function of applied stress (forcelarea) for specimen A, loaded as an embedded film within the gripper tool prototype with a rubber compliant layer and titanium foil.

\subsection{Conclusions}

The NASA RRM marman ring gripper tool was used as a high load, high abrasion, metal-on-metal contact, harsh environment example of potential space servicing tasks requiring force and spatial awareness information during grappling tasks. The actual gripper tool was not available for alteration and lab testing, so a full scale prototype was fabricated which could be machined and tested. Embedding was performed by end milling a cavity into the gripper tool prototype and inserting a thick-film sensor package, silicone rubber compliant layer, and containing the contents with a titanium foil overlay.

Characterization of each embedded sensor constituent (thick-film sensor, rubber compliant layer, and titanium foil) revealed details about the role of item. The bare sensor film experienced a small response with excessive noise. The addition of a compliant silicone layer reduced noise, and slightly increased the sensor response. After adding the metal foil, point forces were more evenly distributed across the rubber compliant layer, and were well distributed over the sensor surface. This full, three-part, embedded sensor gave repeatable results with little negligible noise and drift. 


\section{Chapter 7 - Conclusions and future work}

\subsection{Sensor composition}

\subsubsection{Conclusions}

The design of a three phase, porous ceramic-polymer blend, capacitive type sensor composition consists of many variables, each being optimized between various parameters. In this section, some of the primary considerations for the design of a robust, thin-film sensor composition for space servicing applications are summarized.

- Polymer matrix - The compliant polymer used as a matrix for the sensor composition should be free of transition temperatures within, or near $-80 \rightarrow 120^{\circ} \mathrm{C}$. The material should have elastic properties, with a low elastic modulus to promote strain. Polydispersity of the polymer blend should be very low, reducing viscoelastic effects and promoting controlled volatility (low outgassing) of the material. For this reason, use of organic modifiers was not recommended. Upon curing the polymer matrix should cross link, providing a complex architecture and large molecular weight to prevent reorganization of the microstructure. The matrix should also have a high dielectric breakdown strength, and a high permittivity.

- Ceramic filler - The ceramic filler material is solely intended increase the permittivity of the composite sensor composition. Thus, the primary attribute for the ceramic filler is to have an extremely high permittivity. Despite the low volume loading, it is important for the ceramic filler to have a high dielectric breakdown strength because of the thin nature of the sensor film. The filler should also lack major phase transformations within the working temperature range, which introduce thermal drift in the sensor response. The particle size of the ceramic filler should be minimized to reduce its effect on the elastic modulus, without being reduced to the point of introducing the Curie temperature into the working temperature range through the ferroelectric size effect. Efficient bonding of the filler material with the polymer matrix is achieved through the use of surfactants or modification of the filler surface chemistry. Such bonding is a major contributor to increasing the elastic modulus, though this step is necessary to promote film strength (increasing flexibility and durability). This bonding also plays a key role in reducing movement of ceramic filler particles within the polymer microstructure, which causes drift in the sensor response. 
- Porosity - A porosity is introduced into the sensor material to further reduce the elastic modulus and increase the ability to accurately quantify small stresses. The introduction of closed pores should be avoided, as the enclosed air will seek to vacate upon introduction into the low vacuum space atmosphere. Closed pores can be avoided by preventing agitation during the mixing process, which can introduce air. Interconnected pores can be produced by introducing excessive solvent into the composite ink before the drying process. Careful consideration should be made when selecting an appropriate solvent, as many solvents will persist in the material after the drying process, and compromise the controlled volatility polymers. Generally xylene or toluene will not affect the outgassing properties.

- Volume loading - The precise content of each constituent has been carefully considered both analytically and experimentally. Of the solid loading (ceramic-polymer), a $\sim 10$ vol\% loading of ceramic filler should be used to maximize the bulk material permittivity, while minimizing its effect on the elastic modulus. Of the three phase material, $\sim 25$ vol\% porosity should be used to reduce the elastic modulus, and minimize the reduction in strength and permittivity.

- Thickness - The thickness of the sensor material is the fastest and easiest way to increase the sensor response for a given surface area. It is important to reduce the surface area as much as possible without compromising the dielectric breakdown strength or mechanical strength of the sensor. In this work an optimal sensor thickness of $\sim 9 \mu \mathrm{m}$ was identified to be optimal, though high force, high temperature applications still exceeded the breakdown voltage occasionally.

Additional modifications were examined without further improvements to the sensor response. For example, the introduction of a conductive filler material has been shown in literature to notably increase the permittivity of a bulk material with a volume content just below the conductive percolation threshold. However, the addition of conductive, high modulus materials would reduce the dielectric breakdown strength and increase the elastic modulus of the bulk material, proving counterproductive.

\subsubsection{Future Work}

An extensive and thorough sensor composition was designed, fabricated, and characterized in chapters 35 with excellent results. With the conclusion of the latest design iteration thermal drift continued to persist, introducing error into the sensor response. While this error was improved with the use of PZT ceramic filler rather than BTO, further improvements could be made by evaluating alternate polymer 
matrix materials. For example, NuSil controlled volatility silicone polymers show excellent potential for use as compliant matrix material.

The primary limiting factor in reducing the sensor thickness, and subsequently increasing the capacitance of the sensor, is increasing the breakdown voltage of the sensor material. The BTO filled compositions exhibited a significantly higher breakdown voltage than the PZT filled compositions. For this reason, it is suggested that the filler material be targeted to achieve an increased breakdown strength, despite the small volume fraction of the ceramic filler in the three phase composite. Alterations, such as the introduction of zirconium in BTO, have shown notable increases in the breakdown strength of bulk ceramic materials, and could allow for reduced thickness of the sensor film.

\subsection{Sensor packaging}

\subsubsection{Conclusions}

A number of substrates were evaluated for depositing the sensor composition. When cast in the single micron range, the sensor composition experienced a rate dependent hysteresis. This was caused by pinning of the film on the stiff substrate, which caused the elastic modulus, internal stress, and strain to vary. This error was removed by creating a release agent and removing the sensor film from the substrate, resulting in a free standing film. However, the thin-film was delicate and difficult to handle. Use of a compliant polymer substrate is recommended, such as Teflon ${ }^{\circledR}$ or Kapton ${ }^{\circledR}$ film. These substrate provide a strong, durable substrate without alternating the mechanical properties of the film.

Electrodes were constructed from aluminum, copper, gold, silver, and platinum. Aluminum and copper were found to oxidize, reducing conductivity, and decreased the dielectric breakdown strength of the sensor package because of their decreased work function. Both silver and gold worked well, but platinum electrodes demonstrated the best resilience and dielectric breakdown properties. Electrode deposition should be carried out by DC sputtering, since printing of particulate electrodes decreases electrical and strength properties. Flexibility and conductivity are best optimized with a deposition thickness in the range of $500 \mathrm{~nm}$, where thicker electrodes experience cracking and thinner electrodes begin to lack interconnectivity on the film surface when deposited with DC sputtering.. 


\subsubsection{Future Work}

Many sensor packages were discussed in this work with considerable improvements having been made, though there is still much room for further development. In chapter 2 a process was developed using PVA as a release agent, which allowed for films to be cast using a spin coating process and removed in a hot water bath. Using this method, a new method is proposed to create a sensor package with a total thickness of $\sim 30 \mu \mathrm{m}$, and increased flexibility.

- Release agent: dissolve PVA in water, spin coat onto silicon substrate and dry.

- Substrate: reduce a controlled volatility polymer with xylenes, and spin coat to a thickness of $10 \mu \mathrm{m}$.

- Electrode: Using photolithography processes, deposit a $500 \mathrm{~nm}$ platinum electrode pattern.

- Sensor: Using a mayer rod, cast the desired sensor composition to a thickness of $9.5 \mu \mathrm{m}$.

- Boundary layer: Heat a substrate and place it on the spin coater vacuum chuck, increasing the speed to sufficiently overcome gravitational forces with centrifugal inertia. Reduce the viscosity of the substrate polymer with xylenes and apply after the vacuum chuck has reached the necessary speed. With proper optimization of the polymer viscosity and spinning speed, a very thin layer could be adhered above the porous sensor composition, preventing subsequent electrode deposition from entering the pores.

- Electrode: Using photolithography processes, deposit a $500 \mathrm{~nm}$ platinum electrode pattern.

- Substrate: reduce a controlled volatility polymer with xylenes, and spin coat to a thickness of $10 \mu \mathrm{m}$.

- Releasing: Soak the substrate in a hot water sonication bath until the PVA is dissolved.

The Huntsman polymers were not wet by AZ 5214 E IR photoresist, even with assistance from HMDS, and thus this process was abounded. Identifying an alternative polymer which adheres to the photoresist could allow for the realization of this design. 


\subsection{Embedding}

\subsubsection{Conclusions}

Embedding of the sensor package provided excellent stress distribution without compromising sensitivity. With metal-on-metal contact the bare sensor packaged experienced a small change in capacitance with excessive noise in the signal. This was caused by a combination of point concentrations on the sensor face, and parallel loading directly between the metal plates (offset from the sensor face). After the addition of a rubber compliant layer and a metal foil cover, a small hillock was introduced to promote contact over the sensor face (preventing parallel loads offset from the sensor face) and point loads were distributed across the sensor face. The result was a significant improvement in the sensitivity of the sensor, and a large reduction in signal noise.

\subsubsection{Future Work}

As previously discussed, the compliant rubber silicone layer was protruded from the aluminum gripper prototype a small amount. This design concept was intended to protect the sensor package from being overloaded, as the compliant layer would displace into the milled cavity below the surface of the metal gripper tool, and additional stress would then be transferred to the metal gripper. However, the minimally compressive nature of the silicone rubber caused material to expand under compression through Poisson's ratio, making contact with the surrounding sidewalls of the cavity. After making contact on all axes the silicone had not been fully compressed into the cavity, and overload protection was not achieved.

This design should be improved by allowing room in the axial plane for deformation from Poisson's ratio, or by selecting a compliant layer with a higher compressibility. After observing the excellent load distribution properties of the titanium film, it is suggested that additional high modulus materials be added to the embedded design. A thin layer of metallic or ceramic material between the foil and compliant layer could further distribute point concentrations.

An additional embedded design has also been proposed, which replaces the need for a metal foil or a stiff underlying layer for stress distribution. This design could not be used in the marman ring gripper tool because of strict geometrical requirements in the grasping surface for the specific task. The concept includes a pixelated gripper, where metallic pixels are installed directly into the metal gripper tool. A cross sectional view of a single embedded sensor is shown in figure 77, showing the metallic pixel with 
underlying compliant layer and sensor package. Note that the pixel extends beyond the edges of the compliant layer, where small steps in the gripper tool are machined. This design provides the same overload protection which was presented in chapter 5. As the compliant layer is compressed, a metal-onmetal contact is achieved to prevent further compression. This design will experience the same shortcoming as was discussed in chapter 5, where strain introduced in the axial plane through Poisson's ratio could cause the compliant layer to achieve an incompressible state.
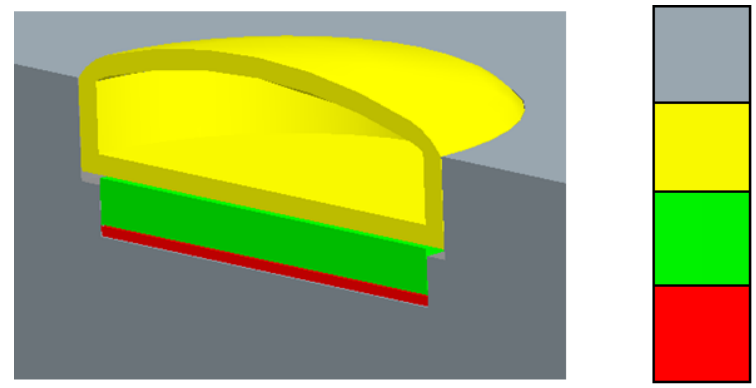

Gripper Tool

Metallic Pixel

Compliant Material

Sensor Package

Figure 77. Cross sectional view of proposed embedding design for pixelated metallic contact points in a gripper tool, providing distribution of point forces and protection from overload through a compliant material. 


\section{Work Cited}

[1] Satellite Industry Association 2011 State of the satellite industry report (Washington, DC: Futron)

[2] Federal Aviation Administration 20112011 Commercial Space Transportation Forecasts

[3] Ellery A, Kreisel J and Sommer, B 2008 The case for robotic on-orbit servicing of spacecraft: Spacecraft reliability is a myth Acta Astronautica 63(5) 632-648

[4] National Aeronautics and Space Administration 2013 NASA facts Robotic Refueling Mission (Greenbelt, MD: NASA GSFC)

[5] Bluethmann W, Rehnmark F, Nguyen T and Aldridge H. 2001 Cooperative manipulation testbed: A facility for space robot control system development Proc. of the 6th i-SAIRAS (Quebec, Canada: Canadian Space Agency)

[6] Rehnmark F, Bluethmann W, Mehling J, Ambrose R O, Diftler M, Chu M and Necessary R 2005 Robonaut: the'Short List'of technology hurdles Computer 38(1) 28-37

[7] Helmick D, Okon A and DiCicco M 2006 A comparison of force sensing techniques for planetary manipulation Aerospace Conf. (IEEE) pp 1-14

[8] Cheung E, Manzo M and McConnell R 1991 Using Capaciflectors for ORU Docking Proc. from the $3^{\text {rd }}$ Ann. Conf. on Intelligent Robotic Systems for Space Exploration (IEEE) pp 76-80

[9] Fricke S, Friedberger A, Seidel H and Schmid U. 2012 A robust pressure sensor for harsh environmental applications. Sensors and Actuators A: Physical.184 16-21

[10] Murphy D G and Deacon S A 2011 Manned GEO satellite servicing mission environmental effects measurements study NASA Report (Hampton, VA: NASA Langley Research Center) pp $1-63$

[11] Petkov M 2003 The effects of space environments on electronic components NASA JPL Report pp $1-28$

[12] Vecchi F, Freschi C, Micera S, Sabatini,A M, Dario P and Sacchetti R 2000 Experimental evaluation of two commercial force sensors for applications in biomechanics and motor control $5^{\text {th }}$ Ann. Conf. of Int. FES.

[13] Yousef H, Boukallel M and Althoefer K 2011 Tactile sensing for dexterous in-hand manipulation in robotics - a review Sensors and Actuators A: Physical 167(2) 171-187

[14] Wang S Y 2004 A finite element model for the static and dynamic analysis of a piezoelectric bimorph International Journal of Solids and Structures 41(15) 4075-4096 
[15] Smith, Charles S. "Piezoresistance effect in germanium and silicon." Physical review 94.1 (1954): 42.

[16] Tufte, O. N., P. W. Chapman, and Donald Long. "Silicon diffused-element piezoresistive diaphragms." Journal of Applied Physics 33.11 (1962): 3322-3327.

[17] Malliaris, Angelos, and D. T. Turner. "Influence of particle size on the electrical resistivity of compacted mixtures of polymeric and metallic powders." Journal of Applied Physics 42.2 (1971): 614-618.

[18] Bueche, F. "A new class of switching materials." Journal of Applied Physics 44 (1973): 532.

[19] ～Harden, John Charles. "PRESSURE PSI." U.S. Patent No. 3,875,434. 1975.

[20] Janzen, Jay. "On the critical conductive filler loading in antistatic composites." Journal of Applied Physics 46.2 (1975): 966-969.

[21] Hierold, C., et al. "Low power integrated pressure sensor system for medical applications." Sensors and Actuators A: Physical 73.1 (1999): 58-67.

[22] Dargahi, Javad, and Siamak Najarian. "Advances in tactile sensors design/manufacturing and its impact on robotics applications-a review."Industrial Robot: An International Journal 32.3 (2005): 268-281.

[23] Kane, Bart J., Mark R. Cutkosky, and Gregory TA Kovacs. "A traction stress sensor array for use in high-resolution robotic tactile imaging." Microelectromechanical Systems, Journal of 9.4 (2000): 425-434.

[24] Vecchi, Fabrizio, et al. "Experimental evaluation of two commercial force sensors for applications in biomechanics and motor control." 5th Ann. Conf. of Int. FES. 2000.

[25] Yousef, Hanna, Mehdi Boukallel, and Kaspar Althoefer. "Tactile sensing for dexterous in-hand manipulation in robotics-A review." Sensors and Actuators A: Physical 167.2 (2011): 171-187.

[26] Puers R 1993 Capacitive sensors: when and how to use them Sensors and Actuators A: Physical 37 93-105

[27] Ashruf C M A 2002 Thin flexible pressure sensors Sensor Review 22(4) 322-327

[28] Brignell J E, White N M and Cranny A W J 1988 Sensor applications of thick-film technology Solid-State and Electron Devices (IEEE Proceedings I) 135(4) 77-84

[29] Arshak K I, McDonagh D and Durcan M A 2000 Development of new capacitive strain sensors based on thick film polymer and cermet technologies Sensors and Actuators A: Physical 79(2) 102-114 
[30] Newnham R E, Skinner D P and Cross L E 1978 Connectivity and piezoelectric-pyroelectric composites Materials Research Bulletin 13(5) 525-536

[31] S.C. Mannsfeld, B.C. Tee, R.M. Stoltenberg, C.V.H. Chen, S. Barman, B.V. Muir, A.N. Sokolov, C. Reese, and Z. Bao (2010). Highly sensitive flexible pressure sensors with microstructured rubber dielectric layers. Nature materials, 9(10), pp. 859-864.

[32] J. Dargahi, M. Kahrizi, N.P. Rao, and S. Sokhanvar (2006). Design and microfabrication of a hybrid piezoelectric-capacitive tactile sensor. Sensor Review, 26(3), pp. 186-192.

[33] D. Ha, W.N. de Vries, S.W. John, P.P. Irazoqui, and W.J. Chappell (2012). Polymer-based miniature flexible capacitive pressure sensor for intraocular pressure (IOP) monitoring inside a mouse eye. Biomedical microdevices, 14(1), pp. 207-215.

[34] K.F. Lei, K.F. Lee, and M.Y. Lee (2012). Development of a flexible PDMS capacitive pressure sensor for plantar pressure measurement. Microelectronic Engineering, 99, pp. 1-5.

[35] Y. Wei, R. Torah, K. Yang, S. Beeby, and J. Tudor (2012). Screen printed capacitive freestanding cantilever beams used as a motion detector for wearable sensors. Procedia Engineering, 47, 165-169.

[36] S. Laflamme, M. Kollosche, J.J. Connor, and G. Kofod (2012). Robust Flexible Capacitive Surface Sensor for Structural Health Monitoring Applications. Journal of Engineering Mechanics. 139(7), pp. 879-885.

[37] H. Luo, and S. Hanagud (1999). PVDF film sensor and its applications in damage detection. Journal of Aerospace Engineering, 12(1), pp. 23-30.

[38] A. Safari (1994). Development of piezoelectric composites for transducers. Journal de Physique III, 4(7), pp. 1129-1149.

[39] A. Seema, K.R. Dayas, and J.M. Varghese (2007). PVDF-PZT-5H composites prepared by hot press and tape casting techniques. Journal of applied polymer science, 106(1), pp. 146-151.

[40] M. Dietze, and M. Es-Souni (2008). Structural and functional properties of screen-printed PZT-PVDF-TrFE composites. Sensors and Actuators A: Physical, 143(2), pp. 329-334.

[41] M. Wegener, and K. Arlt (2008). PZT/P (VDF-HFP) 0-3 composites as solvent-cast thin films: preparation, structure and piezoelectric properties. Journal of Physics D: Applied Physics, 41(16)

[42] R. Igreja, and C. J. Dias (2004). Analytical evaluation of the interdigital electrodes capacitance for a multi-layered structure. Sensors and Actuators A: Physical, 112(2), pp. 291-301. 

Rocha (2011). Nanocomposite flexible pressure sensor for biomedical applications. Procedia Engineering, 25, pp. 140-143.

[44] M.X. Zhou, Q.A. Huang, and M. Qin (2005). Modeling, design and fabrication of a triplelayered capacitive pressure sensor. Sensors and Actuators A: Physical, 117(1), pp. 71-81.

[45] Z. Ahmad, A. Prasad, and K. Prasad (2009). A comparative approach to predicting effective dielectric, piezoelectric and elastic properties of PZT/PVDF composites. Physica B: Condensed Matter, 404(20), pp. 3637-3644.

[46] T. Yamada, T. Ueda, and T. Kitayama (1982). Piezoelectricity of a high-content lead zirconate titanate/polymer composite. Journal of Applied Physics, 53(6), pp. 4328-4332.

[47] A. K. Zak,W.C. Gan, W. H. Majid, M. Darroudi, and T.S. Velayutham (2011). Experimental and theoretical dielectric studies of PVDF/PZT nanocomposite thin films. Ceramics International, 37(5), pp. 1653-1660.

[48] R. Ali, D. Roy Mahapatra, and S. Gopalakrishnan (2004). An analytical model of constrained piezoelectric thin film sensors. Sensors and Actuators A: Physical, 116(3), pp. 424437.

[49] Y. Shen, N. Xi, W.J. Li, and J. Tan (2003, July). A high sensitivity force sensor for microassembly: design and experiments. In Advanced Intelligent Mechatronics, 2003. AIM 2003. Proceedings. 2003 IEEE/ASME International Conference on (Vol. 2, pp. 703-708). IEEE.

[50] Mannsfeld, Stefan CB, et al. "Highly sensitive flexible pressure sensors with microstructured rubber dielectric layers." Nature materials 9.10 (2010): 859-864.

[51] Someya, Takao, et al. "A large-area, flexible pressure sensor matrix with organic field-effect transistors for artificial skin applications." Proceedings of the National Academy of Sciences of the United States of America 101.27 (2004): 9966-9970.

[52] Takei, Kuniharu, et al. "Nanowire active-matrix circuitry for low-voltage macroscale artificial skin." Nature materials 9.10 (2010): 821-826.

[53] Jenkins, Jerald M., and M. M. Lemcoe. Problems associated with attaching strain gages to titanium alloy Ti--6Al--4V. No. N-77-17431; NASA-TM-X-56044; H-970. National Aeronautics and Space Administration, Edwards AFB, CA (USA). Dryden Flight Research Center, 1977.

[54] Schmitz, Alexander, et al. "Methods and technologies for the implementation of large-scale robot tactile sensors." Robotics, IEEE Transactions on 27.3 (2011): 389-400.

[55] Bluethmann, William, et al. "Robonaut: A robot designed to work with humans in space." Autonomous Robots 14.2-3 (2003): 179-197. 
[56] Chang, Wen-Yang, et al. "Flexible electronics sensors for tactile multiscanning." Review of Scientific Instruments 80 (2009): 084701.

[57] Luheng, Wang, Ding Tianhuai, and Wang Peng. "Influence of carbon black concentration on piezoresistivity for carbon-black-filled silicone rubber composite." Carbon 47.14 (2009): 31513157.

[58] Stübler, N., J. Fritzsche, and M. Klüppel. "Mechanical and electrical analysis of carbon black networking in elastomers under strain." Polymer Engineering \& Science 51.6 (2011): 1206-1217.

[59] Wang, Peng, and Tianhuai Ding. "Creep of electrical resistance under uniaxial pressures for carbon black-silicone rubber composite." Journal of materials science 45.13 (2010): 3595-3601.

[60] Dargahi, Javad, Siamak Najarian, and Bin Liu. "Sensitivity analysis of a novel tactile probe for measurement of tissue softness with applications in biomedical robotics." Journal of materials processing technology 183.2 (2007): 176-182.

[61] Bouchet, J., A. A. Roche, and P. Hamelin. "Internal stresses, Young's modulus and practical adhesion of organic coatings applied onto 5754 aluminium alloy." Thin Solid Films 355 (1999): 270-276.

[62] Murphy D G and Deacon S A 2011 Manned GEO satellite servicing mission environmental effects measurements study NASA Report (Hampton, VA: NASA Langley Research Center) pp $1-63$

[63] Petkov M 2003 The effects of space environments on electronic components NASA JPL Report pp 1-28

[64] Vecchi F, Freschi C, Micera S, Sabatini,A M, Dario P and Sacchetti R 2000 Experimental evaluation of two commercial force sensors for applications in biomechanics and motor control $5^{\text {th }}$ Ann. Conf. of Int. FES.

[65] Yousef H, Boukallel M and Althoefer K 2011 Tactile sensing for dexterous in-hand manipulation in robotics - a review Sensors and Actuators A: Physical 167(2) 171-187

[66] Wang S Y 2004 A finite element model for the static and dynamic analysis of a piezoelectric bimorph International Journal of Solids and Structures 41(15) 4075-4096

[67] Puers R 1993 Capacitive sensors: when and how to use them Sensors and Actuators A: Physical 37 93-105

[68] Ashruf C M A 2002 Thin flexible pressure sensors Sensor Review 22(4) 322-327

[69] Brignell J E, White N M and Cranny A W J 1988 Sensor applications of thick-film technology Solid-State and Electron Devices (IEEE Proceedings I) 135(4) 77-84 
[70] Arshak K I, McDonagh D and Durcan M A 2000 Development of new capacitive strain sensors based on thick film polymer and cermet technologies Sensors and Actuators A: Physical 79(2) 102-114

[71] Newnham R E, Skinner D P and Cross L E 1978 Connectivity and piezoelectric-pyroelectric composites Materials Research Bulletin 13(5) 525-536

[72] Sun W 2006 Size effect in barium titanate powders synthesized by different hydrothermal methods Journal of applied physics 100(8) 83503-83503

[73] Dietze M and Es-Souni M 2008 Structural and functional properties of screen-printed PZTPVDF-TrFE composites Sensors and Actuators A: Physical 143(2) 329-334

[74] Kobayashi Y, Kosuge A and Konno M 2008 Fabrication of high concentration barium titanate/polyvinylpyrrolidone nano-composite thin films and their dielectric properties Applied Surface Science 255(5) 2723-2729

[75] Wang Y G, Zhong W L and Zhang P L 1994 Size effects on the Curie temperature of ferroelectric particles Solid state communications 92(6) 519-523

[76] Jaccard C, Kanzig W and Peter W 1953 Das Verhalten von kolloidalen Seignetteelektrika I, Kaliumphosphat $\mathrm{KH}_{2} \mathrm{PO}_{4}$ Helv. Phys. Acta 26 521-544

[77] Mészáros R, Varga I and Gilányi T 2005 Effect of polymer molecular weight on the polymer/surfactant interaction The Journal of Physical Chemistry B 109(28) 13538-13544

[78] Teoh M M and Chung T S 2009 Micelle-like macrovoids in mixed matrix PVDF-PTFE hollow fiber membranes Journal of Membrane Science 338(1) 5-10

[79] Yang M H, Hou S Y, Chang Y L and Yang A M 2006 Molecular recoiling in polymer thin film dewetting Physical review letters 96(6) 066105.1-066105.4

[80] Shaw T M, Suo Z, Huang M, Liniger E, Laibowitz R B and Baniecki J D 1999 The effect of stress on the dielectric properties of barium strontium titanate thin films Applied physics letters 75(14) 2129-2131

[81] Devonshire A F 1954 Theory of ferroelectrics Advances in physics 3(10) 85-130

[82] Rouse Jr P E 1953 A theory of the linear viscoelastic properties of dilute solutions of coiling polymers The Journal of Chemical Physics 21 1272-1280

[83] Lin Y H 1987 Number of entanglement strands per cubed tube diameter, a fundamental aspect of topological universality in polymer viscoelasticity Macromolecules 20(12) 3080-3083

[84] Dalnoki-Veress K, Forrest J A, De Gennes P G and Dutcher J R 2000 Glass transition reductions in thin freely-standing polymer films: A scaling analysis of chain confinement effects Le Journal de Physique IV 10(7) 221-226 
[85] Wasserman S H and Graessley W W 1996 Prediction of linear viscoelastic response for entangled polyolefin melts from molecular weight distribution Polymer Engineering \& Science 36(6) $852-861$

[86] Montfort J P, Marin G and Monge P 1986 Molecular weight distribution dependence of the viscoelastic properties of linear polymers: the coupling of reptation and tube-renewal effects Macromolecules 19(7) 1979-1988

[87] Vollenberg P H T and Heikens D 1989 Particle size dependence of the Young's modulus of filled polymers: 1. Preliminary experiments Polymer 30(9) 1656-1662

[88] Bao N, Shen L, Srinivasan G, Yanagisawa K and Gupta A 2008 Shape-controlled monocrystalline ferroelectric barium titanate nanostructures: From nanotubes and nanowires to ordered nanostructures The Journal of Physical Chemistry C 112(23) 8634-8642

[89] Suntako R, Laoratanakul P and Traiphol N 2009 Effects of dispersant concentration and pH on properties of lead zirconate titanate aqueous suspension Ceramics International 35(3) 1227-1233

[90] Boutaleb S, Zaïri F, Mesbah A, Naït-Abdelaziz M, Gloaguen J M, Boukharouba T and Lefebvre J M 2009 Micromechanics-based modelling of stiffness and yield stress for silica/polymer nanocomposites International Journal of Solids and Structures 46(7) 1716-1726

[91] Ji X L, Jing J K, Jiang W and Jiang B Z 2002 Tensile modulus of polymer nanocomposites. Polymer Engineering \& Science 42(5) 983-993

[92] Fu S Y, Feng X Q, Lauke B and Mai Y W 2008 Effects of particle size, particle/matrix interface adhesion and particle loading on mechanical properties of particulate-polymer composites Composites Part B: Engineering 39(6) 933-961

[93] Alam P 2010 A mixtures' model for porous particle-polymer composites Mechanics Research Communications 37(4) 389-393

[94] Cassu S N and Felisberti M I 1999 Poly (vinyl alcohol) and poly (vinylpyrrolidone) blends: 2. Study of relaxations by dynamic mechanical analysis Polymer 40(17) 4845-4851

[95] Linares A and Acosta J L 1997 Tensile and dynamic mechanical behaviour of polymer blends based on PVDF European polymer journal 33(4) 467-473

[96] Drzymala J and Wheelock T D 1995 Air agglomeration of hydrophobic particles Iowa State Univ. of Science and Technology (Ames, IA: Iowa State Univ.)

[97] Bechhoefer J, Deng Y, Zylberberg J, Lei C and Ye Z G 2007 Temperature dependence of the capacitance of a ferroelectric material American Journal of Physics 75 1046-1053

[98] Jin B M, Kim J and Kim S C 1997 Effects of grain size on the electrical properties of $\mathrm{PbZr}_{0}$. ${ }_{52} \mathrm{Ti}_{0 .}{ }_{48} \mathrm{O}_{3}$ ceramics Applied Physics A: Materials Science \& Processing 65 53-56 
[99] Ohno T, Suzuki D, Ishikawa K and Suzuki H 2007 Size effect for lead zirconate titanate nanoparticles with PZT (40/60) composition Advanced Powder Technology 18(5) 579-589

[100] Forsbergh Jr P W 1949 Domain structures and phase transitions in barium titanate Physical Review 76(8) 1187-1201

[101] Kinoshita K and Yamaji A 1976 Grain-size effects on dielectric properties in barium titanate ceramics Journal of Applied Physics 47 371-373

[102] Buessem W R, Cross L E and Goswami A K 1966 Phenomenological Theory of High Permittivity in Fine-Grained Barium Titanate Journal of the American Ceramic Society 49 33-36

[103] Buessem W R, Cross L E and Goswami A K 1966 Effect of Two-Dimensional Pressure on the Permittivity of Fine-and Coarse-Grained Barium Titanate Journal of the American Ceramic Society 49 36-39

[104] Arlt G and Hennings D 1985 Dielectric properties of fine-grained barium titanate ceramics Journal of Applied Physics 58(4) 1619-1625

[105] Bell A 1984 Residual stress in BTO from temperature Ferroelectrics 54 147-150

[106] Curecheriu L, Buscaglia M T, Buscaglia V, Zhao Z and Mitoseriu L 2010 Grain size effect on the nonlinear dielectric properties of barium titanate ceramics Applied Physics Letters 97(24) 242909-242909

[107] Pertsev N A, Zembilgotov A G, Hoffmann S, Waser R and Tagantsev A K 1999 Ferroelectric thin films grown on tensile substrates: Renormalization of the Curie-Weiss law and apparent absence of ferroelectricity Journal of Applied Physics 85(3) 1698-1701

[108] Cowie J M G 1991 Polymers: Chemistry \& Physics of modern materials (CRC Press)

[109] Ree M, Chu C W and Goldberg M J 1994 Influences of chain rigidity, in-plane orientation, and thickness on residual stress of polymer films Journal of applied physics 75(3) 1410-1419

[110] Robertson C G, Lin C J, Rackaitis M and Roland C M 2008 Influence of particle size and polymer- filler coupling on viscoelastic glass transition of particle-reinforced polymers Macromolecules 41(7) 2727-2731

[111] Osman M A and Atallah A 2006 Effect of the particle size on the viscoelastic properties of filled polyethylene Polymer 47(7) 2357-2368

[112] Priya L and Job J P 2002 Poly(vinylidene fluoride)/Clay Nanocomposites Prepared by Melt Intercalation: Crystallization and Dynamic Mechanical Behavior Studies Journal of Polymer Science: Part B: Polymer Physics 40 1682-1689

[113] Bleach N C, Nazhat S N, Tanner K E, Kellomäki M and Törmälä P 2002 Effect of filler content on mechanical and dynamic mechanical properties of particulate biphasic calcium phosphatepolylactide composites Biomaterials 23(7) 1579-1585 
[114] El Mohajir B E and Heymans N 2001 Changes in structural and mechanical behaviour of PVDF with processing and thermomechanical treatments. 1. Change in structure Polymer 42(13) $5661-5667$

[115] Sichel E K, Gittleman J I and Sheng P 1978 Transport properties of the composite material carbon-poly (vinyl chloride) Physical Review B 18(10) 5712-5716

[116] Jing X, Zhao W and Lan L 2000 The effect of particle size on electric conducting percolation threshold in polymer/conducting particle composites Journal of materials science letters 19(5) 377-379

[117] Dias C J and Das-Gupta D K 1996 Inorganic ceramic/polymer ferroelectric composite electrets IEEE Transactions on Dielectrics and Electrical Insulation 3(5) 706-734

[118] Newnham R E 1985 Ferroelectric Composites Jpn. J. Appl. Phys.24 16-17

[119] Furukawa T, Ishida K and Fukada E 1979 Piezoelectric properties in the composite systems of polymers and PZT ceramics Journal of Applied Physics 50(7) 4904-4912

[120] J. Dargahi, M. Kahrizi, N.P. Rao, and S. Sokhanvar (2006). Design and microfabrication of a hybrid piezoelectric-capacitive tactile sensor. Sensor Review, 26(3), pp. 186-192.

[121] R. Igreja, and C. J. Dias (2004). Analytical evaluation of the interdigital electrodes capacitance for a multi-layered structure. Sensors and Actuators A: Physical, 112(2), pp. 291301.

[122] A.T. Sepúlveda, F. Fachin, R. Villoria, B.L. Wardle, J.C. Viana, A.J. Pontes, and L.A. Rocha (2011). Nanocomposite flexible pressure sensor for biomedical applications. Procedia Engineering, 25, pp. 140-143.

[123] M.X. Zhou, Q.A. Huang, and M. Qin (2005). Modeling, design and fabrication of a triple-layered capacitive pressure sensor. Sensors and Actuators A: Physical, 117(1), pp. 71-81.

[124] Z. Ahmad, A. Prasad, and K. Prasad (2009). A comparative approach to predicting effective dielectric, piezoelectric and elastic properties of PZT/PVDF composites. Physica B: Condensed Matter, 404(20), pp. 3637-3644.

[125] T. Yamada, T. Ueda, and T. Kitayama (1982). Piezoelectricity of a high-content lead zirconate titanate/polymer composite. Journal of Applied Physics, 53(6), pp. 4328-4332.

[126] A. K. Zak,W.C. Gan, W. H. Majid, M. Darroudi, and T.S. Velayutham (2011).

Experimental and theoretical dielectric studies of PVDF/PZT nanocomposite thin films. Ceramics International, 37(5), pp. 1653-1660. 
[127] R. Ali, D. Roy Mahapatra, and S. Gopalakrishnan (2004). An analytical model of constrained piezoelectric thin film sensors. Sensors and Actuators A: Physical, 116(3), pp. 424437.

[128] Y. Shen, N. Xi, W.J. Li, and J. Tan (2003, July). A high sensitivity force sensor for microassembly: design and experiments. In Advanced Intelligent Mechatronics, 2003. AIM 2003. Proceedings. 2003 IEEE/ASME International Conference on (Vol. 2, pp. 703-708). IEEE.

[129] R. Hill (1963). Elastic properties of reinforced solids: some theoretical principles. Journal of the Mechanics and Physics of Solids, 11(5), pp. 357-372.

[130] R. Hill (1964). Theory of mechanical properties of fibre-strengthened materials: I. Elastic behaviour. Journal of the Mechanics and Physics of Solids, 12(4), pp. 199-212.

[131] J.C. Affdl, and J. L. Kardos (1976). The Halpin-Tsai equations: a review.Polymer Engineering \& Science, 16(5), pp. 344-352.

[132] E.H. Kerner (1956). The electrical conductivity of composite media.Proceedings of the Physical Society. Section B, 69(8), 802.

[133] J.J. Hermans (1967). The elastic properties of fiber reinforced materials when the fibers are aligned. In Proc. K. Ned. Akad. Wet. B (Vol. 70, No. 1, pp. 1-9).

[134] J.E. Ashton, J. C. Halpin, and P.H. Petit (1969). Primer on composite materials: analysis. Technomic Publishing Company.

[135] A.J. Kinloch, and A.C. Taylor, (2003). Mechanical and fracture properties of epoxy/inorganic micro-and nano-composites. Journal of materials science letters, 22(20), pp. 1439-1441

[136] R.M. Spriggs (1961). Expression for effect of porosity on elastic modulus of polycrystalline refractory materials, particularly aluminum oxide. Journal of the American Ceramic Society, 44(12), pp. 628-629.

[137] J.C. Wang (1984). Young's modulus of porous materials. Journal of Materials Science, 19(3), pp. 801-808.

[138] K.K. Phani, and K.S. Niyogi, S. K. (1987). Young's modulus of porous brittle solids.Journal of materials science, 22(1), pp. 257-263.

[139] F.P. Knudsen (1962). Effect of porosity on Young's modulus of alumina. Journal of the American Ceramic Society, 45(2), pp. 94-95.

[140] L.J. Gibson, M.F. Ashby. Cellular solids: structure and properties. Oxford: Pergamon Press; 1988, pp. 120-68. 
[141] J.J. Blaker, V. Maquet, R. Jérôme, A.R. Boccaccini, and S.N. Nazhat (2005). Mechanical properties of highly porous PDLLA/Bioglass ${ }^{\circledR}$ composite foams as scaffolds for bone tissue engineering. Acta biomaterialia, 1(6), pp. 643-652.

[142] A.P. Roberts, and E.J. Garboczi (2001). Elastic moduli of model random threedimensional closed-cell cellular solids. Acta materialia, 49(2), pp. 189-197.

[143] K. Zhang, Y. Ma, and L.F. Francis (2002). Porous polymer/bioactive glass composites for soft-to-hard tissue interfaces. Journal of biomedical materials research, 61(4), pp. 551-563.

[144] O. Ishai, and L.J. Cohen (1967). Elastic properties of filled and porous epoxy composites. International Journal of Mechanical Sciences, 9(8), pp. 539-546.

[145] K. Zhang, Y. Wang, M.A. Hillmyer, and L.F. Francis, L. F. (2004). Processing and properties of porous poly (L-lactide)/bioactive glass composites. Biomaterials, 25(13), pp. 24892500.

[146] F.G. Torres, S.N. Nazhat, S.H. Sheikh Md Fadzullah, V. Maquet, A.R. Boccaccini (2007). Mechanical properties and bioactivity of porous $\mathrm{PLGA} / \mathrm{TiO}_{2}$ nanoparticle-filled composites for tissue engineering scaffolds. Composites science and technology, 67(6), pp. 11391147.

[147] T.L. Weadon, T.H. Evans, E.M. Sabolsky (2013). Ceramic-polymer capacitive sensors for tactile/force awareness in harsh environment robotic applications. Smart Materials and Structures, 22(12)

[148] A.C. Dent, C.R. Bowen, R. Stevens, M.G. Cain, and M. Stewart (2007). Effective elastic properties for unpoled barium titanate. Journal of the European Ceramic Society, 27(13), pp. 3739-3743.

[149] B. Ploss (1998). Influence of poling and annealing on the nonlinear dielectric permittivity of PVDF-TRFE copolymers. Dielectrics and Electrical Insulation, IEEE Transactions on, 5(1), pp. 91-95.

[150] H. Osterberg, J.W. Cookson (1937). The Piezodielectric Effect and Electrostriction in Anisotropic or Isotropic Media. Physical Review, 51(12), pp. 1096-1101.

[151] A.F. Devonshire (1954). Theory of ferroelectrics. Advances in physics, 3(10), pp. 85130.

[152] T.M. Shaw, Z. Suo, M. Huang, E. Liniger, R.B. Laibowitz, and J.D. Baniecki (1999). The effect of stress on the dielectric properties of barium strontium titanate thin films. Applied physics letters, 75(14), pp. 2129-2131.

[153] S. Bhaskar, S.B. Majumder, and R.S. Katiyar (2002). Diffuse phase transition and relaxor behavior in (PbLa) $\mathrm{TiO}_{3}$ thin films. Applied physics letters, 80(21), pp. 3997-3999. 
[154] Beatrix Ploss, Bernd Ploss, F.G. Shin, H.L. Chan, C.L. Choy (2000). Pyroelectric or piezoelectric compensated ferroelectric composites. Applied Physics Letters, 76(19), pp. 27762778.

[155] D. Damjanovic (1998). Ferroelectric, dielectric and piezoelectric properties of ferroelectric thin films and ceramics. Reports on Progress in Physics, 61(9), pp. 1267-1324.

[156] M.J. Haun, E. Furman, S.J. Jang, H.A. McKinstry, and L.E. Cross (1987).

Thermodynamic theory of $\mathrm{PbTiO}_{3}$. J. Appl. Phys. 62

[157] N. Jayasundere, and B.V. Smith (1993). Dielectric constant for binary piezoelectric 0-3 composites. Journal of Applied Physics, 73(5), pp. 2462-2466.

[158] A. Sihvola (2000). Mixing rules with complex dielectric coefficients. Subsurface Sensing Technologies and Applications, 1(4), pp. 393-415.

[159] D.A. Robinson, S.P. Friedman (2001). Effect of particle size distribution on the effective dielectric permittivity of saturated granular media. Water Resources Research, 37(1), pp. 33-40.

[160] T. Bhimasankaram, S.V. Suryanarayana, G. Prasad (1998). Piezoelectric polymer composite materials. CURRENT SCIENCE-BANGALORE-, 74, pp. 967-976.

[161] S.F. Mendes, C.M. Costa, V. Sencadas, J.S. Nunes, P. Costa, R. Gregorio Jr, and S. Lanceros-Méndez (2009). Effect of the ceramic grain size and concentration on the dynamical mechanical and dielectric behavior of poly (vinilidene fluoride) $/ \mathrm{Pb}\left(\mathrm{Zr}_{0.53} \mathrm{Ti}_{0.47}\right) \mathrm{O}_{3}$ composites. Applied Physics A, 96(4), pp. 899-908.

[162] W.R. Tinga, W.A.G. Voss, and D.F. Blossey (1973). Generalized approach to multiphase dielectric mixture theory. Journal of Applied Physics, 44(9), pp. 3897-3902.

[163] L. Rayleigh (1892). LVI. On the influence of obstacles arranged in rectangular order upon the properties of a medium. The London, Edinburgh, and Dublin Philosophical Magazine and Journal of Science, 34(211), pp. 481-502.

[164] J.M. Garnett (1904). Colours in Metal Glasses and in Metallic Films.Proceedings of the Royal Society of London, 73(488-496), pp. 443-445.

[165] V.D. Bruggeman (1935). Berechnung verschiedener physikalischer Konstanten von heterogenen Substanzen. I. Dielektrizitätskonstanten und Leitfähigkeiten der Mischkörper aus isotropen Substanzen. Annalen der Physik, 416(7), pp. 636-664.

[166] A.H. Sihvola, and J.A. Kong (1988). Effective permittivity of dielectric mixtures. Geoscience and Remote Sensing, IEEE Transactions on, 26(4), pp. 420-429.

[167] X.L. Ji, J.K. Jing, W. Jiang, and B.Z. Jiang (2002). Tensile modulus of polymer nanocomposites. Polymer Engineering \& Science, 42(5), pp. 983-993. 
[168] I.M. Wienk, R.M. Boom, M.A.M. Beerlage, A.M.W. Bulte, C.A. Smolders, and H. Strathmann (1996). Recent advances in the formation of phase inversion membranes made from amorphous or semi-crystalline polymers. Journal of membrane science, 113(2), pp. 361-371.

[169] M. Arous, I.B. Amor, A. Kallel, Z. Fakhfakh, and G. Perrier (2007). Crystallinity and dielectric relaxations in semi-crystalline poly (ether ether ketone). Journal of Physics and Chemistry of Solids, 68(7), pp. 1405-1414.

[170] T. Furukawa, M. Date, E. Fukada, Y. Tajitsu, A. Chiba (1980). Ferroelectric behavior in the copolymer of vinylidenefluoride and trifluoroethylene. Jpn. J. Appl. Phys, 19(2), pp. L109L112.

[171] P. Ueberschlag (2001). PVDF piezoelectric polymer. Sensor Review, 21(2), pp. 118126.

[172] C. Basceri, S.K. Streiffer, A.I. Kingon, and R. Waser (1997). The dielectric response as a function of temperature and film thickness of fiber-textured $(\mathrm{Ba}, \mathrm{Sr}) \mathrm{TiO}$ thin films grown by chemical vapor deposition. Journal of Applied Physics, 82, pp. 2497-2504.

[173] Dang, Z-M., Y. Shen, and C-W. Nan. "Dielectric behavior of three-phase percolative NiBaTiO 3/Polyvinylidene fluoride composites." Applied Physics Letters 81.25 (2002): 4814-4816.

[174] Ni, Hai, et al. "Cycloaliphatic polyester-based high-solids polyurethane coatings: II. The effect of difunctional acid." Progress in organic coatings 45.1 (2002): 49-58.

[175] Malave, Vincent, et al. "Uniquely Customized Ultra-Low-Outgassing Silicones to Reduce Contamination." Journal of Spacecraft and Rockets 48.2 (2011): 235-239.

[176] Vaccaro, J. F. "Unique UV Urethane Adhesives, Coatings and Encapsulants." RADTECH REPORT 17.2 (2003): 48-53.

[177] Jia, Q. X., Z. Q. Shi, and W. A. Anderson. "BaTiO 3 thin film capacitors deposited by rf magnetron sputtering." Thin Solid Films 209.2 (1992): 230-239.

[178] Dias C J and Das-Gupta D K 1996 Inorganic ceramic/polymer ferroelectric composite electrets IEEE Transactions on Dielectrics and Electrical Insulation 3(5) 706-734

[179] Vollenberg P H T and Heikens D 1989 Particle size dependence of the Young's modulus of filled polymers: 1. Preliminary experiments Polymer 30(9) 1656-1662

[180] Zhong, W. L., et al. "Phenomenological study of the size effect on phase transitions in ferroelectric particles." Physical Review B 50.2 (1994): 698.

[181] K. Ishikawa, K. Yoshikawa, N. Okada: Size effect on the ferroelectric phase transition in PbTiO3 ultrafine particles, Phys. Rev. B 37, 5852-5855 (1988) 327

[182] Deng, Yuan, et al. "Hydrothermal synthesis and characterization of nanocrystalline PZT powders." Materials Letters 57.11 (2003): 1675-1678. 
[183] Wang, Xin Yu, et al. "Dispersion and aging behavior of $\mathrm{BaTiO}_{3}$ and PZT in water." Materials research bulletin 35.14 (2000): 2555-2563.

[184] Welberry, T. R., et al. "Monte Carlo simulation study of diffuse scattering in PZT, Pb (Zr, Ti) O3." Metallurgical and materials transactions A 41.5 (2010): 1110-1118.

[185] Golan, Yuval, Lev Margulis, and Israel Rubinstein. "Vacuum-deposited gold films: I. Factors affecting the film morphology." Surface science 264.3 (1992): 312-326.

[186] Shaw, T. M., et al. "The effect of stress on the dielectric properties of barium strontium titanate thin films." Applied physics letters 75.14 (1999): 2129-2131.

[187] Head, David A., Alex J. Levine, and F. C. MacKintosh. "Deformation of cross-linked semiflexible polymer networks." Physical review letters 91.10 (2003): 108102.

[188] Bharadwaj, R. K., et al. "Structure-property relationships in cross-linked polyester-clay nanocomposites." Polymer 43.13 (2002): 3699-3705.

[189] Beeby, S. P., A. Blackburn, and N. M. White. "Processing of PZT piezoelectric thick films on silicon for microelectromechancial systems." Journal of Micromechanics and Microengineering 9.3 (1999): 218.

[190] Jeon, Yongbae, Jaeshik Chung, and Kwangsoo No. "Fabrication of PZT thick films on silicon substrates for piezoelectric actuator." Journal of electroceramics 4.1 (2000): 195-199. [191] Scott, J. F. "High-dielectric constant thin films for dynamic random access memories (DRAM)." Annual review of materials science 28.1 (1998): 79-100. 